\title{
EXPANDING PLANNING PUBLIC PARTICIPATION OUTREACH THROUGH SOCIAL NETWORKING
}

\author{
A Thesis \\ presented to \\ the Faculty of California Polytechnic State University, \\ San Luis Obispo
}

\author{
In Partial Fulfillment \\ of the Requirements for the Degree \\ Master of City and Regional Planning
}

by

Wesley Harris

June 2011 
(C) 2011

Wesley Brian David Harris

ALL RIGHTS RESERVED

COMMITTEE MEMBERSHIP 


\section{TITLE: $\quad$ EXPANDING PLANNING PUBLIC PARTICIPATION OUTREACH THROUGH SOCIAL NETWORKING}
AUTHOR:
Wesley Brian David Harris
DATE SUBMITTED:
June 2011

COMMITTEE CHAIR:

Umut Toker, Ph.D.

Assistant Professor

COMMITTEE MEMBER: $\quad$ Hemalata C. Dandekar, Ph.D.

Department Head

COMMITTEE MEMBER: James David

City of San Luis Obispo, Assistant Planner 


\begin{abstract}
EXPANDING PLANNING PUBLIC PARTICIPATION OUTREACH

THROUGH SOCIAL NETWORKING
\end{abstract}

Wesley Brian David Harris

Public participation is not a form of civic responsibility that it once was. With not only fewer people taking part in the public participation process, there is a trend towards an older (45 years and older) group of residents that come to such meetings or workshops. Plans, such as Specific Plans or General Plans often take years to implement and require all generations to give feedback on what is needed for the future. Additionally, within the last decade, there has been a rise in social media websites such as Facebook and Twitter. These websites emerged as informal virtual places for friends to connect, but have slowly evolved into a tool for businesses, and more importantly, government to connect with constituents. This study explores the relationship between the decline of public participation with findings to support the reasons residents do not take part in the process, and the rise of social media as a tool for engagement with findings to support how cities nationwide use Facebook. Social media provides a two-way form of communication between the community and the local government which aides in promoting genuine participation. Additionally, social media allows for efficient outreach and noticing of meetings or public workshops. As opposed to newspaper or website noticing, websites such as Facebook allow for local governments to target a specific audience by location, age, or interests. Findings indicate that although many cities developed a Facebook Page to engage the "younger generation", all ages became fans of the City operated Facebook Page. In addition, the findings show that the true potential of Facebook as a participatory tool have not been discovered. cities are developing their own ways of using it as a tool as there is no formal best practices manual for City planning departments. The findings of this study have provided the necessary information to develop a best practices manual for planning practitioners to utilize. The manual provides information on developing a Facebook Page as well as the implications of the technology.

Keywords: Public Participation, Outreach, Social Media, Youth, Digital Natives, Civic Engagement, Facebook, Twitter 


\section{ACKNOWLEDGMENTS}

I would like to first and foremost acknowledge my wife, Jessica, for the sacrifices you've made to get me where I am today. You have motivated me to aspire to greater accomplishments and not stop until I reached my goals. You believed in me in the times I didn't believe in myself and for that I am thankful to have you by my side for the rest of my life.

To my grandmother, Diane, thank you for making all this possible financially. You've allowed me to pursue a higher education in times of financial hardship. You've always had faith in me and I promise to use my education to make you proud and, perhaps, pay you back one day.

Dr. Umut Toker, you have been a pleasure to work with and have supported my research from the beginning. Thank you for being available as well as being adaptive to changes in my research and suggesting new ways of thinking about the problem.

Dr. Hemalata Dandekar, thank you for providing your insight and asking critical questions that shaped this research. You provided another perspective that I was unaware of and I am grateful for that.

James David, I appreciate all the work you have done and the time commitments you have made to shape this research. Your suggestions have led to a focused study that provides critical information to partitioning planners such as yourself. 


\section{TABLE OF CONTENTS}

Page

LIST OF FIGURES .............................................................................. ix

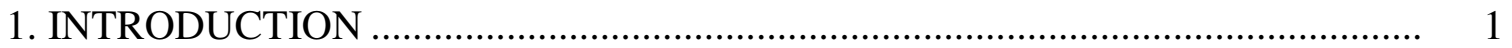

2. EXISTING LITERATURE …................................................................. 4

2.2 Background of public participation in the United States .......................... 4

2.3 Advantages and disadvantages of current public participation practices ...................................................................... 8

2.4 Apathy and cynicism within youths in the public participation process ..................................................... 10

2.5 Social networking and public participation ........................................ 13

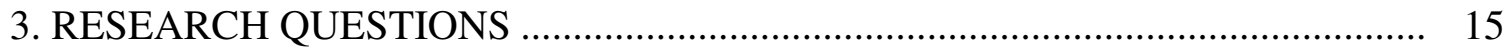

4. METHODOLOGY …................................................................................. 16

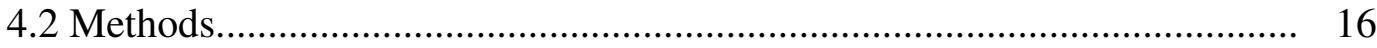

4.3 Instrumentation ...................................................................... 17

4.3.2 Case study .............................................................. 17

4.3.3 Public surveys .................................................................. 18

4.3.4 City interviews .............................................................. 18

4.3.5 Participant observations ................................................ 19

4.4 Sampling strategy...................................................................... 19

4.5 Glossary of terms ..................................................................... 21

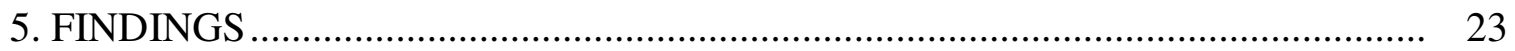

5.2 Planning Commission surveys .......................................................... 23

5.2.2 Planning Commission meeting - April 5, 2011 _.................... 23

5.3 General public surveys................................................................. 24

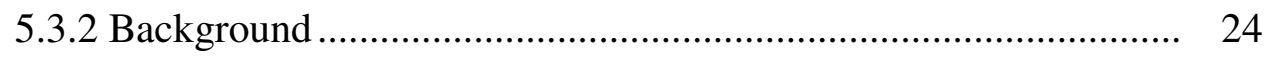

5.3.3 Survey session 1 observations - May 9, 2011 2:00-3:30 PM...... 26 
5.3.4 Survey session 2 observations - May 11, 2011 5:00-6:00 PM.... 27

5.3.5 Compiled survey data ...................................................... 27

5.4 Atascadero Facebook page .......................................................... 34

5.4.2 Page development ........................................................ 34

5.4.3 Profile photo................................................................... 35

5.4.4 Wall and commenting policy ............................................. 36

5.4.5 Information tab............................................................. 37

5.4.6 Terms of use ............................................................... 39

5.4.7 Photos........................................................................... 40

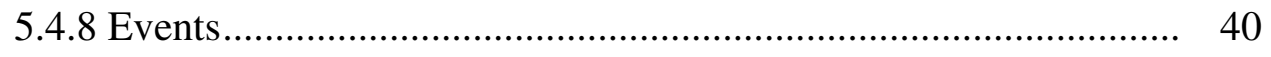

5.4.9 Additional tools............................................................. 44

5.4.10 PDF/Doc formats ...................................................... 44

5.4.11 Discussion ................................................................... 44

5.4.12 Twitter.................................................................... 42

5.4.13 Page insight .............................................................. 42

5.4.14 Promotion....................................................................... 46

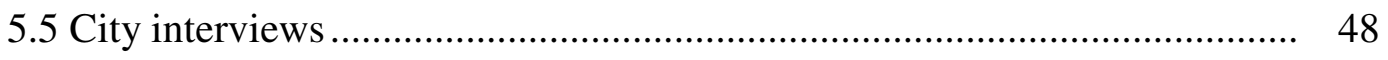

5.5.2 Background ............................................................... 48

5.5.3 Primary goal of using Facebook ........................................ 49

5.5.4 Deciding to use Facebook ................................................... 50

5.5.5 Operation of the page ..................................................... 51

5.5.6 Internal protocol for operating the page................................ 52

5.5.7 Issues and concerns with the page ...................................... 53

5.5 .8 Obtaining goals ...................................................... 54

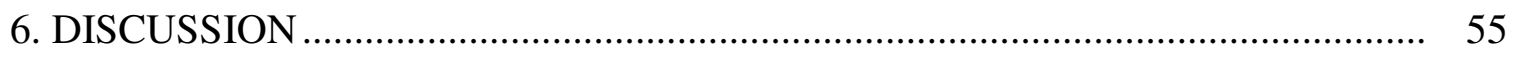


6.2 Outreach ................................................................................. 56

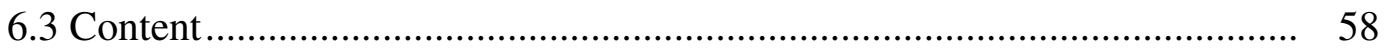

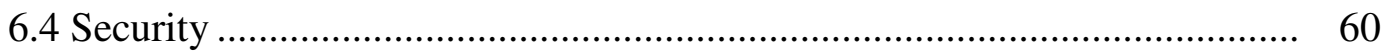

6.5 Department/office ..................................................................... 63

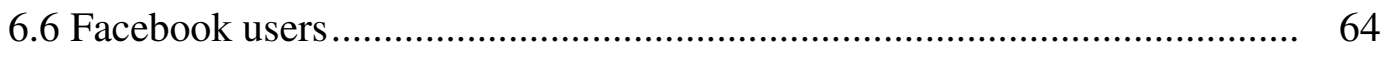

6.7 Time-cost benefits............................................................................. 65

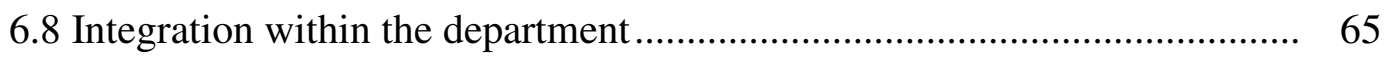

7. CONCLUSIONS AND IMPLICATIONS FOR PLANNERS ............................ 67

7.2 Review of research questions....................................................... 68

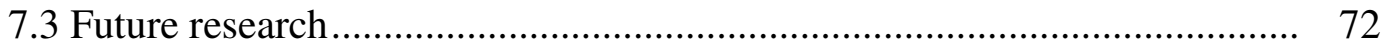

7.4 Best practices manual for planning practitioners .................................. 76

Five Key Recommendation................................................... 76

Why use Facebook? ........................................................... 78

Creating a Facebook page ......................................................... 79

Steps to creating a page ........................................................... 82

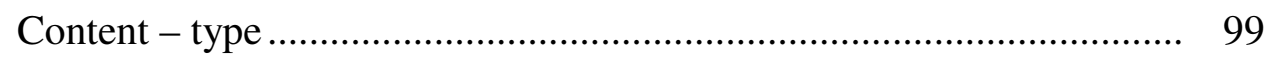

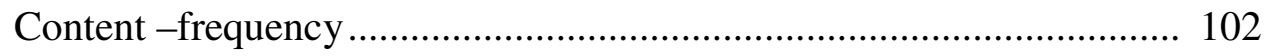

Internal terms of use protocol ............................................... 103

Promotion and advertising .................................................... 106

Badges and social plugins ...................................................... 111

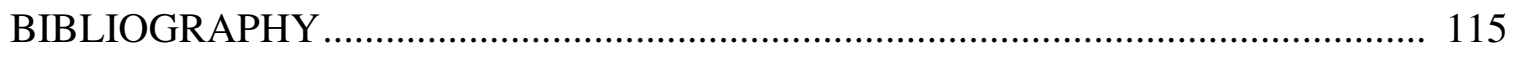

\section{APPENDICES}

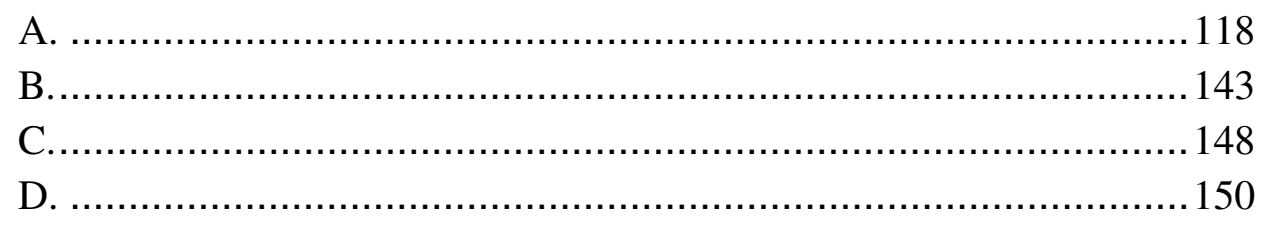




\section{LIST OF FIGURES}

Figure $\quad$ Page

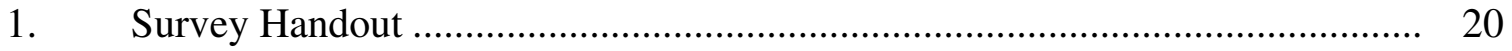

2. $\quad$ Age of Participants with Trend Line ........................................................ 28

3. Comparison of Participants Age and Sex ............................................ 28

4. Notifications of Public Meetings .............................................................. 30

5. Attendance at Public Meetings ............................................................. 31

6. Reasons for Not Attending Public Workshops or Hearings .......................... 32

7. Comparison of Residents on Facebook users and Public

Hearing/Workshop Attendance............................................................... 32

8. Non Facebook Users by Age ............................................................. 33

9. Facebook Users by Age ....................................................................... 33

10. Profile Photo Selected for the Atascadero Facebook Page ............................. 36

11. Active Users - 2/25/11 - 5/26/11 .......................................................... 43

12. Daily Active Users Breakdown - 2/25/11 - 5/26/11 .................................. 43

13. Daily Likes $-2 / 25 / 11$ - 5/26/11 ......................................................... 44

14. Total Likes $-2 / 25 / 11$ - 5/26/11 _........................................................... 44

15. Gender and Age Demographics ......................................................... 45

16. Page Views $-2 / 25 / 11$ - 5/26/11 .......................................................... 46

17. The Facebook Badge is Located on Every Page of the City's Website ............ 47

18. City of Atascadero's Social Media Page ....................................................... 48

19. Facebook Login Page ....................................................................... 83

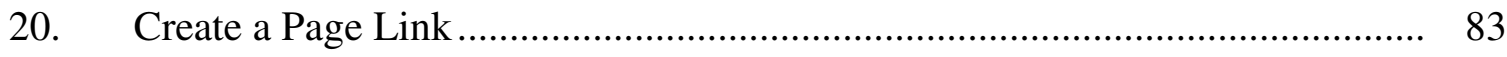

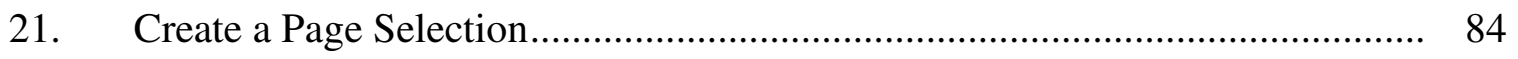

22. Organization Subcategories …......................................................... 85 
23. Get Started Page ............................................................................. 86

24. Example of an Iconic Photograph for the Profile Picture .................................. 87

25. Edit Page and Edit Info Locations ............................................................... 87

26. Facebook Page Settings/Navigation Bar....................................................... 88

27. Manage Permissions Settings …………………...................................... 90

28. Basic Information Settings..................................................................... 92

29. Administrator Privileges .......................................................................... 93

30. Application Selection........................................................................... 94

31. Sample Photo Album, City of Atascadero ………………………................... 95

32. Creating an Event ............................................................................. 96

33. Event Details as Viewed by Fans................................................................. 96

34. Discussion Section ................................................................................... 97

35. Example of Terms of Use Language …………......................................... 98

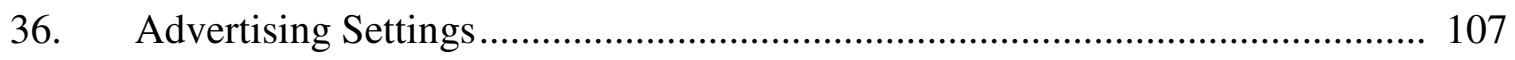

37. Step 1 of Advertising on Facebook - Designing the Ad .................................. 108

38. Step 2 of Advertising on Facebook -Targeting and real time Estimate Reach

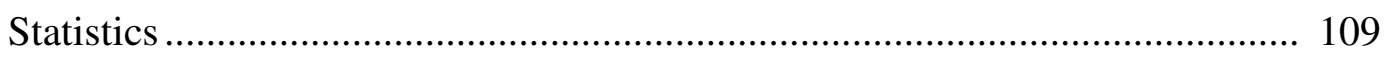

39. Step 3 of Advertising on Facebook - Campaigns, Pricing, and Scheduling .... 110

40. Social Plugins on the City of Atascadero Website .......................................... 112

41. City of Long Beach Website Homepage with Clear and Identifiable

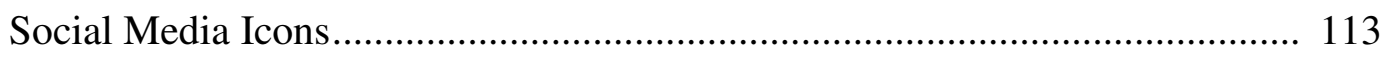

42. Lists of Social Media Pages Categorized by Media Type ………….................. 113 


\section{Introduction}

Public participation is the key process that connects community members with the decision making power of governments and organizations. The United States government is based on the simple notion of "government of the people, by the people, for the people..." (Lincoln, 1863). The U.S. is a nation of freedom of speech and democracy, which in turn means that to run efficiently there needs to be a stable connection between the people and the government (or agencies) to properly run as a country. Therefore, researching the area of participation is a relevant and important topic in planning. This is because planning is essentially dependent on public input and participation to ensure that government and organizations are providing for the community. Public planning requires, by law, the inclusion of public participation within planning decisions. This is found most commonly in planning commission meetings as well as public workshops. Community members in turn provide important information for planners that might not otherwise have been obtained through typical observations or survey data. This creates a feedback loop of community members expressing their views to planners and decision makers, and consequently the decision makers approval or denial of projects based on the community's input. It is the information that residents provide that planners utilize when designing physical space as well as relevant policies. Beyond the legal requirements of engaging the public in participation, the practice of planning allows for legitimacy within the government and provides an arena for communication.

This study will focus on a single case study within the county of San Luis Obispo to explore the various methods of outreach, and an assessment of who typically attends workshops and public hearings. The research will look into two aspects of the local 
planning public participation process. The first aspect is the outreach methods conducted by local governments for announcing participation events such as public hearings or planning workshops. The study will explore how different residents are reached and if there is a bias towards one form of outreach over another. For example, does the City send out required noticing through the local newspaper only, and if so, what are the demographic characteristics of that population which reads the newspaper? Conversely, if the noticing is only online, who has access to the website address in addition to a computer and internet connection? The second approach suggests new outreach methods to capture a greater number of residents. This can be further broken down into formally or informally giving residents notice through social media websites such as Facebook or Twitter and/or providing public input opportunities for those who cannot physically attend a meeting. This study will look at the relationship between attendance and median age and thus suggest new or improved outreach methods to capture a wider community audience across the age spectrum.

It must be noted that this study focuses on users of digital technology and those who have access to this technology in California. It is assumed that there is a segment of the population who may not have access to the internet and thus this study is not applicable to that population. Other researchers are encouraged to look into the area of public participation outreach through other means of distribution. In essence this study is focusing on bringing those who are actively using social networking websites and/or smartphone "apps" to learn about public participation events. In addition, the main concern of this study is to engage residents to attend meetings. Meeting content is itself 
disregarded in this study. This to allow a clear and concise focus on the outreach aspect of planning alone. 


\section{Existing Literature}

The topic of public participation in planning is relatively large and thus for the purpose of this study must be divided into two distinct but connected areas of research. The first area of concern is outreach methods, and determining how invitations are dispersed throughout the community. As stated earlier this study is only concerned only with bringing residents to the meeting and is not looking at the content of the meetings. The second area of concern builds upon the first area and focuses on why those who are aware of public meetings choose not to engage. Public participation is widely acknowledge for its many important benefits and functions (Irvin and Stansbury, 2004, Webler et al, 2001) and therefore should be an area of focus with regard to the types of outreach methods. In addition, the public participation process is a legally required component of planning and as discussed later, there are forms of "pseudoparticipation" and "genuine participation" (Deshler \& Sock, 1985) that occur because of this requirement. The concern here is that the community inherently has the power to decide the future of development in their city, so why is there a decline in public participation? (Berman, 2007). The current research indicates a number of explanations for community members not participating in recent years and thus gives insight into new areas of focus with regard to engaging residents (Berman, 2007; Reed, 2001).

\subsection{Background of public participation in the United States}

Participation is an important process for the United States as it closely follows the American ideal of democracy. Involving any and all residents in the public decision making process provides for many benefits above and beyond the obvious transparency of government. The planning profession is one of the few professions that seeks to shape 
the built environment and what intervention can or cannot do on their property. This form of government does not coincide with the typical American ideology of a free market. Planners have the ability to raise or lower property values through zoning and this is one of the reasons that understanding the public participation process is important to the practice.

The Ralph M. Brown Act (CA Gov. Code, § 54950), passed by California legislature in 1953 was a major statute that provided for the public's right to attend and participate in meetings. The Brown Act added to the transparency of government and ensures the public has a say in decision. The League of California cities explains that two major aspects of the Brown Act that have not changed since it was first in enacted in 1953 are both the Legislature's intent to codify that public commissions and boards exist to serve the people:

In enacting this chapter, the Legislature finds and declares that the public commissions, boards, and councils and the other public agencies in this State exist to aid in the conduct of the people's business. It is the intent of the law that their actions be taken openly and that their deliberations be conducted openly."

"The people of this State do not yield their sovereignty to the agencies which serve them. The people, in delegating authority, do not give their public servants the right to decide what is good for the people to know and what is not good for them to know. The people insist on remaining informed so that they may retain control over the instruments they have created.

And the Legislature's mandate that all legislative meetings of local agencies be open to the public:

All meetings of the legislative body of a local agency shall be open and public, and all persons shall be permitted to attend any meeting of the legislative body of a local agency, except as otherwise provided in this chapter. (League of California cities, 2007, p. 2)

The Brown Act therefore provides the basis for public participation in California and covers a broad spectrum of members and meeting types. Although this Act was initially intended for typical face-to-face meetings, it has evolved with technologies and the world of telecommunications through amendments and updates. The main concern is that 
members of a legislative body have new methods of digital communication such as cell phones and laptops that may be used in meetings which may give rise to the perception that private discussions are occurring through back channels even as the public meeting is being held. The League of California cities explains,

\begin{abstract}
New communication technologies present new Brown Act challenges. For example, common email practices of forwarding or replying to messages can easily lead to a serial meeting prohibited by the Brown Act, as can participation by members of a legislative body in an Internet chatroom or blog dialogue. Communicating during meetings using electronic technology (such as laptop computers, personal digital assistants, or cellular telephones) may create the perception that private communications are influencing the outcome of decisions; some state legislatures have banned the practice. On the other hand, widespread cablecasting and web streaming of meetings has greatly expanded public access to the decision-making process. $(2007$, p.3)
\end{abstract}

The last sentence provides insight into the benefits of new technologies and how they have "expanded public access to the decision-making process." Therefore the Brown Act provides a basis for public participation in California and gives reason for conducting meetings that involve the public as a means of engagement and as such gives citizens a sense of control in their local government.

Sherry Arnstein states, "there is a critical difference between going through the empty ritual of participation and having the real power needed to affect the outcome of the process." (1969, p.337). Arnstein is responsible for the popular article on the eight rungs of the public participation ladder titled A Ladder of Citizen Participation. She explains that there are various levels of public participation involvement that range from "Citizen Control", to "Manipulation". This is essentially explaining the balance of participation from a top down approach of government dictating planning to a bottom up approach of community members taking full control of the community they are in. The evaluation of the two case studies in this research will include comparisons to Arnstein's ladder to find similarities and differences between level of participation and attendance. 
Although a relatively old article, it provides a basis of comparison to that epitome of the public participation movement, the Civil Rights Movement of the 1960s.

Beyond Arnstein, Henry Sanoff suggests that "a new pragmatic approach to participation has emerged, one that no longer views participation as defined by Arnstein's (1969) categorical term for "'citizen power"” (2000, p.8). Essentially Sanoff is building on Arnstein's study of the top 3 of the 8 rungs of the citizen participation ladder, and suggesting that the public participation process has evolved. Deshler and Sock (1985) provide the notion of two levels of participation which are 1) Pseudoparticipation and 2) Genuine Participation (as cited in Sanoff, 2000, p.8). These two levels essentially are at the heart of the public participation process where pseudoparticipation is a top down form where government informs the public in a one-way conversation. The other level, Genuine Participation, involves the public in a partnership with the planning agency and allows their input to be directly related to whatever plan they are working on. Although it can be suggested that many people feel that public participation is important, Sanoff presents three lines of reasoning for its importance as follows:

1. Participation involves people in the decision making processes and in turn increases their trust within government and organizations, making it more likely that they will be open and accepting of the plans and decisions. This in turn will assist in working within the established systems when seeking other solutions to other problems.

2.Participation provides people with a say in the design and decision making process in order to improve and streamline plans, decisions, and service delivery.

3. Participation promotes a sense of community by bringing people together who share common goals. (2000, p.9-10).

Sanoff provides groundwork for the reasoning behind the participation process and suggests that the more involved people are the more readily projects will be passed. What this means is that fewer people are likely to hold up a project through litigation or 
other stalling tactics due to the fact they were not involved in the public participation process. This, although is not a definite result, provides incentives for agencies and organizations to involve the constituents throughout the process.

\subsection{Advantages and disadvantages of current public participation practices}

Public participation is an important aspect of local government in the United States and can be attributed to increased appreciation of the larger community among the public as well as create a better decision-making process through public preference (Irvin and Stansbury 2004; Stivers 1990; Oldfield 1990; Box 1998). With a streamlined process, governments can essentially make better informed decisions that might otherwise be halted by individuals or groups against an idea or project. Irvin and Stansbury (2004) indicate that there are several advantages of public participation including;

1. Increased education in the area of policy and decision making,

2. Public suasion and essentially allowing for a "more cooperative public" (p.56).

3. Avoiding litigation costs is an important aspect of public participation because it provides for a forum of knowledge and understanding of a project and allow input from citizens prior to big decision and so a trusting bond is created. Litigation is less likely with a well-informed public although Coglianese (1997) suggests that collaboration in regulatory negotiations did not necessarily result in less cases of litigation, and that true litigation rates may have been exaggerated.

Although Irvin and Stansbury suggested additional advantages to community participation, they also indicate the disadvantages. While there may be a well-known and widely agreed upon need for public participation, there are associated disadvantages that 
make the process difficult and, as Irvin and Stansbury (2004) suggest, may not be "worth" it. Their study presents interesting concepts with regard to the negative impacts of public participation including:

1. Cost for accommodating community-participation groups compared to a single decision maker from within the agency. Irving and Stansbury suggest that the work of a single administrator may come to the same decision as the community group chose, but having taken far less time to come to that decision.

2. Lack of representation is common in larger communities where only 10-20 members of the community participate from the entire city. Essentially they represents a very small portion of the population and "unless they are known to represent a constituency, there are no guarantees that each citizen participant is influential in his or her community" (Irvin and Stansbury, 2004, p.58).

3. Complacency is one of the major problems with citizen-participation processes. In addition to Irvin and Stansbury (2004) suggesting that residents feel alienated from the public affairs process, Evan Berman (1997), produced a study on cynicism and public participation in planning and found that many community members feel disconnected from their government and have somewhat of a distrust. Creating new ways to rebuild that trust are essential for solving complacency.

4. As Irvin and Stansbury (2004) discuss, lack of authority is another issue with public participation that impacts the processes. The process itself allows residents to believe they have a say and an impact in the final decision. Community members may not respond well to thinking their decisions will be implemented 
only to find them ignored or taken under advisement. There is the chance of resentment developing over time with continual public participation processes and nothing to show for it after the process.

This study used the findings of Irving and Stansbury and apply it towards the analysis of the findings. With well-known and agreed upon benefits of public participation there are still disadvantages to the process. Effective means of public participation is one of the ways to reengage the public into civic life and limit the increasing apathy among residents.

\subsection{Apathy and cynicism within youths in the public participation process}

Current research shows there is a lack of public participation within the planning profession and that this may outwardly indicate a level of apathy on behalf of the community members, that there are in fact other methods that residents are engaged in. (Reed, 2001; Berman, 1997; Eliasoph, 1998) The typical public participation event is either in the form of a public hearing where the community sits in rows of seating and watches decision makers discuss planning topics with both planning staff and developers and/or designers of the project in question. The public normally has three minutes to approach a podium and speak to the decision makers, planning staff, and those who are proposing the project. Due to the fact that only one person can speak at a time, the time limit on speaking allows for everybody to speak and also hold the public meeting within a reasonable amount of time and also for the public meeting to be held within a reasonable amount of time. This form of public participation, which Sanoff describes as pseudo, is "nonparticipatory" and essentially "people's participation wherein control of a 
project rests with administrators is pseudoparticipation. Here the level of participation is that of people being present to listen to what is being planned for them" (2000, p.8). It is implied that public hearings, although are a form of public participation, do not implicitly involve residents in the form of decision making. As Sanoff stated, people are there to listen to what is being planned for them as opposed to being planned by them. It can be concluded that people may consider their views unimportant and useless within the planning process. This leads to a sense of cynicism and distrust for government which breaks down the participation process and has potential for negative effects on projects and policies in the future.

Evan Berman conducted a study titled "Dealing with Cynical Citizens" (1997) that focused on why people in general are cynical towards government and the planning process. Berman's study indicated that only $46.3 \%$ of those interviewed agreed that government officials are honest, and $43.0 \%$ agreed that government fulfills its promises. This in turn means a majority of respondents found that government officials are dishonest and that government does not fulfills on its promises. Berman indicates that "Citizens see government taxing them, charging them fees and fining them. While granting special favors to special interests" (Berman 1997, p.106) This can be seen in the typical public hearing where community members are at the mercy of a time limit while those working on the project may have ample time to fully disclose the project details to the decision makers with or without help of planning staff (who benefit from permitting and other fees if the project is approved). Berman's study found that "cynicism is present in about one-third of all cities with populations over 50,000" (p. 111), essentially meaning that there is a level of distrust within many cities in the United States. Residents 
therefore either do not attend public participation events such as public hearings or attend but are cynical about the process which in turn may change their outlook on the meeting. Over time cynical community members may become apathetic to the process as they perceive their voice is not being heard through the traditional public hearing meeting. Apathy therefore leads to less opposition to government by the majority of residents and a greater sense of disengagement.

Currently young people are becoming less engaged in government politics and activism when compared to the heyday of activism in the mid to late 20th century. This occurrence is on a global level as Harris et al. (2010) indicates in their study on apathy within youths where they state "it is commonly accepted today that many young people in a globalized world cease to see the relevance of state-based politics or state-oriented activism and are no longer finding meaning in or opportunities for traditional modes of affiliation and participation (2010, p.10). Although their study was within Australia, there are commonalities among the youths within the United States and Australia. Harris et al. (2010) found that there was a disconnect "between 'real' politics and its official language and young people's 'everyday' concerns and their informal discursive and practical repertories" (2010, p.20). Within this study, youths did not engage in the typical sense of public participation or political affiliation, but instead engaged in informal participation. Informal participation such as recycling, donating money to a cause, signing a petition, discussing politics, or making a statement through art, writing or music were the top responses that Harris et al. received in their study. Essentially youths are trying to find ways to promote change in the political system while avoiding the formal ways of participating. This is important to planning because as an arena for public participation, it 
is very much a political profession where youths distrust the typical political machine. The study focused on ways to enhance public participation in youths and essentially suggested the Internet as one tool for engaging youths.

The Internet was widely used by those within this study, where $94 \%$ had access to the internet at home. The study found that the internet "offered a virtual forum for the expression and debate of social and political concerns; in other words, it filled the space once occupied by civic associations, political groups and other participatory organizations where young people of a previous generation were able to join with others and engage in democratic practice" (p. 26). This change in methods of participation is very important to the study of planning outreach and engagement. If planning does not in one way or another engage in new technologies that are widely used by young community members, then the participation process may not as effective as possible.

\subsection{Social networking and public participation}

Social networking had become increasingly popular within the last decade with the creation of profile-based sites such as MySpace and Facebook. These websites allow users to create profiles with personal information but also to broadcast their "status" or become parts of online groups which include topics of anything from television shows, music bands and automobiles to, more importantly, political groups and campaigns. In addition to multifaceted sites such as Facebook and MySpace, there is Twitter. Twitter allows users to follow other users and see only status updates. This method of communication is limited to 140 characters' or less, which in turn creates short succinct messages from people to anyone in the world. This form of information exchange provides for a rapid sharing of information as well as defining relationships between 
people and groups of people. In the study conducted by Richard Waters, et al. (2009) titled Engaging stakeholders through social networking: How nonprofit organizations are using Facebook there is data to support the validity of Facebook as a tool for nonprofit organizations. This study found that the nonprofit organizations used the discussion board feature of Facebook the most (74\%) as a dissemination method. It was found that many of the organizations did not use the full potential of the website for their cause and instead focused on using the website as a broadcasting vehicle for their cause.

The potential power of social networking for planning agencies is great as there are opportunities for typically free outreach as well as the opportunity for two way communication. Waters (2009) suggested that the nonprofit organizations are beginning to experiment with the different features of the Facebook site to engage their stakeholders. Engaging stakeholders through communication on one website provides for increased participation efforts and possibly a decrease in citizen cynicism or apathy towards government. Planning organizations, cities, counties, and state agencies can all create Facebook profiles as a form of outreach and engage residents in a form of participation as they have an opportunity to write on the profile, their views and opinions of upcoming events or projects. This in turn opens up a new form of civic engagement and participation for community members that may not otherwise come to public meetings or workshops. 


\section{Research Questions}

This study focuses on two distinct areas of public participation and attempts to make a connection between them both. It is one thing to inform the public of a meeting or workshop, but it is something else to have them actively come to a public meeting. Generally participation consists of community members physically attending the meeting/workshop in order to speak their mind about the particular topic. The same participants may show up to every community event independent of the topic to speak their mind and use the time to express their various concerns, or there may be a different participatory group for each meeting in which feedback may be inconsistent when compared to the same person(s) giving input at each meeting. Through case studies, this study will attempt to answer the following research questions:

1) How can Facebook be used as an efficient means of community outreach/participation?

2) Is there a difference between age groups as age relates to the use of Facebook for public participation?

3) What are the ways in which users access Facebook to be informed about city activities?

4) Can city residents who do not attend public meetings be informed via Facebook?

5) What are the practices of those cities that use Facebook as an outreach and public participation tool?

Quality data collection and methodology is needed to effectively answer these questions. This age range was chosen as it includes young adults who have a stake in their community. 


\section{Methodology}

The primary objective of this study is to explore the relationship, if any, between age range and public participation in planning . The study will focus on the use of Facebook specifically and how cities utilize the software. Comparisons between cities as well as the case study will provide insight and direction for practicing planners who wish to use Facebook or social media in general as an additional outreach/education tool.

\subsection{Methods}

Data collection focused on two aspects of public participation and the use of Facebook. The first, focused on public participation events such as Planning Commission to determine the number of people who participate and their ages. Research was also expanded to determine the decisions made by those who were not at the observed public participation events. This included surveying the general public to understand if they participate in public planning as well as their age. This method of research focused on the user side of public participation and was compared to the planning side via a case study and formal interviews with cities. Robert Yin (2003) suggests the use of the case study to understand the "how" or "why" programs work within government agencies. Yin justifies the use of the case study in addition to surveys as a way explore and gain a better understanding of the item being researched. Yin explains:

if you want to know "what" the outcomes of a new government program had been, you could answer this question by doing a survey or by examining economic data, depending upon the type of program involved. Questions - such as "How many clients did the program serve?" "What kinds of benefits were received?" "How often were different benefits produced?" - all could be answered without doing a case study. But if you needed to know "how" or "why" the program had worked (or not), you would lean toward either a case study or a field experiment. (2003, p.10) Yin's example corresponds with the type of research and the proposed outcome of this study. In addition to a case study, surveys (both formal and informal) were developed to explore how Facebook is used and what the implications are for planners. 


\subsection{Instrumentation}

Two instruments were implemented for this study, 1) case studies, and 2) surveys. These instruments provided insight and information into the public realm of participation as well as the planning realm of outreach and education.

\subsubsection{Case Study}

The City of Atascadero, California was the primary case study on the development, launch, and maintenance of a Facebook Page. In addition to creating and launching the page, observations were made of the content posted and feedback from fans. This information provided primary data of opportunities and constraints with regard to using Facebook as a tool for public participation. Multiple meetings were held with the city to ensure the page was acceptable and that it followed the basic best practices that were initially developed for this study. Access was granted to observe the administrative side of the Facebook page in order to gain access to the "Insight" feature which is Facebook's own use and demographic information.

Opportunities and constraints were observed via the Insight feature as well as discussion with the administrator form the city. Quantitative data collected included total number of fans ("likes" of the page), age cohorts, sex, active users, and both active page views and unique page views. This data presented a profile of residents using the page and provided an understanding of their behavior. Qualitative data collected included meetings with the administrator to determine any issues with operating the page such as, responding to comments, creating new content, frequency of updating, and resources needed. 


\subsubsection{Public Surveys}

Surveys were conducted that provided information on the public realm of participation within the city of Atascadero. The surveys were designed for the general public of Atascadero to gain an understanding of their public participation behavior and to determine if they are Facebook users.

Respondents were asked if they have attended an Atascadero planning public participation event such as a workshop or public hearing. Those that indicated that they did take part in such an event were then asked how they heard of the event and how often they attend such meetings. Conversely, for those who indicate that they do not or have not attended a public hearing or city workshop were asked what was the main reason for not attending? All respondents were then asked if they are a Facebook user as well as their age and sex. This survey was designed to be short and concise to respect respondents' time as the surveys were conducted at the exit of a grocery store.

\subsubsection{City Interviews}

cities within the contiguous United States were interviewed via phone or email to determine their involvement with Facebook and social media in general. Appendix A contains the interview transcripts and State of interviewee.

The goals, decision to use Facebook, process of operating the Page, internal terms of use, and major issues of the Page were determined in short interview. Goals provided information on why they city decided to use Facebook, and information on who championed the idea provided an understanding of the resources needed to create a page. Additionally, information on operating the page was very important. This information included security concerns as well as any issues with producing content, responding to 
comments, and the administrator's responsibilities. Information provided by the respondents was compiled, analyzed and used (in addition to other resources) within the best practices section of this report.

\subsubsection{Participant Observations}

Observations were conducted at Atascadero Planning Commission meetings to determine how many people attend and their age. An additional survey was designed for a public participation event, such as a city Planning Commission city Council meeting. This survey was similar to the one designed and administered outside of the grocery store. However an additional question asking what type of media outreach will most likely have them attend a public meeting or workshop. Surveys were made available on the table next to the meeting agenda with associated signage. Potential respondents were not directly asked to take the surveys as to respect the meeting and wishes of the city. Observations were taken of the meeting to understand who attends, such as the general public, concerned citizens, or the applicant of an agenda item.

\subsection{Sampling Strategy}

Case Study - The City of Atascadero, California was selected as a case study because of the existing interest to move forward with social networking. The city was interested in the creation of a Facebook page and this study was the catalyst to the move forward. As this study focused on age, the demographics of Atascadero were considered when selecting the case study. Atascadero's median age is 42 years old with $25.4 \%$ of the population between the ages of 15 and 34 years old (United States Census, 2010). With the city available to update and maintain the page, Atascadero presented itself as a major candidate for this study. 
Public Surveys - The public surveys were conducted within the city of Atascadero at one of four major supermarkets within the city. The surveys were conducted on two separate occasions at the same location. The first survey session was on May 9, 2011 at 2:00-3:30 PM. The second survey session was on May 11, 2011 5:00-6:00 PM. These times provided for those residents who may be working a typical 8:00AM - 5:00PM job and as such one survey was conducted during this time and the other was after. The location was a random selection of the four major supermarkets within city as all four are within five miles of each other. Additionally respondents were asked to participate in the survey as they exited the store with the option of walking to their car while responding to the questions. If respondents indicated that they were a Facebook user, then a small piece of paper with information about the Atascadero City Facebook Page was presented to them (Figure 1).

\section{facebook.}

Find the City of Atascadero at: www.facebook.com/planningatascadero

Figure 1 Survey Handout

City Interviews - cities within the contiguous United States were selected State by State through a simple selection process. cities were listed in descending order of the most populated for up to the top five most populated cities in each state. The official city website was accessed and if it was determined that they had an official Facebook Page, then they were contacted to conduct the interview. For cities that did not have an official Facebook Page, the next most populated city was selected. cities were given the option to 
respond via email or telephone. A full list of the cities contacted can be found in Appendix B.

\subsection{Glossary of Terms}

This study will use a variety of terms native to Facebook and social media. This section is a dedicated glossary of the terms commonly used in Facebook.

Administrator - A page administrator or more commonly referred to as an "Admin" is the person in control of the content and settings of a Page. Administrators have the ability to make other Facebook users administrators for a group or a Page.

Applications - These are small programs either created by Facebook or third-party developers that provide additional sections or services to a page. E.g. the PDF/Document viewer application allows the administrator to incorporate PDF's or other documents within the Page.

Badges - Badges have been recently renamed to Social Plugins are icons or large graphical links that connect external websites with the Facebook Page. Badges can contain information such as the title of the Page and a thumbnail of the profile picture. Badges are created by the user and use HTML code to be inserted into external webpages.

Facebook Page - Pages are dedicated to organizations, businesses, brands, and public figures for Facebook users to contact and interact directly in an unofficial and public manner. A Page is not a profile, although they look very similar.

Facebook Profile/User Account - People who want to be a part of the Facebook network must create a profile that represents their basic information and interests. A profile is required to create and become the administrator of any Facebook Page (see: Facebook Page).

Fan - A person who has joined or "liked" a page because they like what the page represents.

FMBL - Facebook Markup Language is a variation of HTML (Hypertext Markup Language) and allows for developers to create applications.

Friend - A person who has joined a personal profile. 
Group - A group is not a profile or a Page. It is a semi-profile page for groups of people such as bands, companies and other organizations. Groups are differentiated by administrative rights and features of the page.

Hits - Hits or Page Hits is a term used for how often a webpage is accessed. (See Page Views for the Facebook equivalent)

Impression - Any time content is accessed or loaded into a web browser's memory, it creates an impression which is recorded and displayed by Facebook's Insight feature (see Insight)

Insights - This is Facebook's own type of web page analysis. This feature is only available to Facebook Pages and tracks various information such as page views, interactions, fans, and demographics.

Like - This is a major feature of Facebook and appears internally within profiles, Pages, and status updates. In addition the "like" button can be found on external websites that link back to a Page or profile. The purpose of this feature is to allow other users to see who agrees with the content posted, whether it be a comment, video, photo, link, or something else.

News Feed - This is a section of Facebook that provides a compiled list of the most recent or most relevant updates that friends are posting. Additionally content from Pages is presented in the news feed.

SPAM - This is unrelated information that can be posted on the Facebook wall or directly messaged to the administrator. This information can be annoying or harmful if it contains links or information to unsafe webpages.

Tabs - Tabs have recently been removed from the top of the status update page to the left side of the page. Tabs are used interchangeably with "section" of the Facebook Page and represent different areas or applications of a Page.

Tag - This is marking a photo or a status update with another person or Page.

Thumbnail - Photos that are reduced in size for the purpose of representing the photos are called thumbnails. Generally thumbnails can be selected to reveal the original size of the photo.

Wall - The wall is the main area for sharing and communication. This is where the administrator posts information and where fans can respond to also post content. 


\section{Findings}

\subsection{Planning Commission Surveys}

In order to better understand how residents hear about planning related issues, data was collected at the City of Atascadero Planning Commission meetings. In addition to firsthand observations, surveys were created specifically for participants of the Planning Commission meetings. In addition, of the ten Planning Commission meetings scheduled from January 2011 to May 2011, four were cancelled. However, data was still collected/observed on April 5, 2011 to determine the level of participation.

\subsubsection{Planning Commission Meeting - April 5, 2011}

The City of Atascadero held a regularly scheduled Planning Commission meeting at 7:00 PM at City Hall that was open to the public. The short surveys and informed consent forms were made available next to the printed agendas on the table at the entrance prior to the meeting. The meeting had the following two agenda items:

\section{APPROVAL OF ACTION MINUTES OF THE REGULAR PLANNING COMMISSION MEETING ON MARCH 15, 2011. \\ 4. PLN 2011-1396, TREE REMOVAL PERMIT FOR REGIO PLACE (OAK GROVE)}

These agenda items are not considered contentious and require either a low level or no level of public participation. The meeting started on-time at 7:00 with three people from the public seated at the beginning. Of the three people from the public, two of them were the applicant for item number 2 above. The other person was a resident who did not speak and was not otherwise associated with the project or the applicants. The Planning Commission encouraged the applicants to approach the podium to speak for clarification of the project as well as additional background information. There was no other public input on this item or at any other time during the meeting. Of the three residents none of 
them participated in the survey, yielding no data. The meeting indicated that there is a low level of participation that correlates with low level interest items such as tree removal. Although this data is not conclusive, it assists in understanding the public participation process and levels of participation.

\subsection{General Public Surveys}

General surveys were used to understand if residents of Atascadero are aware of and attend public meetings and/or public workshops for the city. In addition, participants were asked if they are on Facebook to understand any correlation between Facebook users and public meeting participants. Surveys were administered on two separate occasions at two different times of the day. 79 people participated in the survey over the two-day course. In addition to conducting surveys, observations were conducted and analysis is provided at the end of this section.

\subsubsection{Background}

The Albertson at $8200 \mathrm{El}$ Camino Real in Atascadero was chosen as the location to conduct both surveys. To ensure there were no duplicate surveys as well as respect the time of the participant, surveys were taken of those exiting the building rather than entering. In addition to respect the time of participants as well as avoid blocking the exit, participants were given the option to walk while taking the survey. These measures were taken to ensure that participants felt comfortable in taking the time to answer the questions. An important note was how to initially address potential participants as they exited the building. Between the exit doors and the beginning of the parking lot was an area roughly 15 feet long. This meant that potential participants had to be addressed and give consent to a survey in a relatively short amount of time. Through informal observations (as a resident of Atascadero) it has been observed that this particular 
Albertsons has a lot of solicitors from typical Boy or Girl Scouts, to voter registration and other surveys. It was therefore important to succinctly express the surveys affiliation with Cal Poly, the non-political nature of the survey, and approximate time to complete.

Below is the typical introduction used to gain trust and participation:

"Hello, I am a student from Cal Poly working on my master's thesis. Do you have time for a very short survey on public participation?"

In addition announcing the affiliation with Cal Poly, the surveyor wore an official Cal Poly tee-shirt to assist with the association of the institution. Potential participants that were obviously busy on the phone or assisting their children in any way were not surveyed as to respect their time. All other potential participants were questioned with consent and either pulled to the side to avoid congestion at the exit or walked with to their vehicle. It was observed that when asked "Are you on Facebook" that some participants hesitated. Although it was not said, it became apparent that participants were unsure if the surveyor would solicit information through Facebook by garnishing their personal information. Therefore the question was immediately amended to include "please note, none of your personal information will be collected and this is only for demographic information". After this amendment, it was observed that participants felt comfortable and confident in saying "yes" if they were on Facebook. For those who said "yes" to being on Facebook, they were given a small piece of paper with information on the official Atascadero City Planning Facebook Page. This information was given for the primary purpose of advertising the city page and to encourage more people to join or become a fan of the page. The advertisement was handed to the participant with information about why they should visit the page. One example used was an explanation that the Facebook page featured new and upcoming noteworthy projects around 
Atascadero and includes a photo section called "From Concept to Construction". This information was presented to participants to give them an idea of what to expect from a governmental Facebook page and increase interest.

\subsubsection{Survey Session 1 Observations - May 9, 2011 2:00-3:30 PM}

The first survey session took place at 2:00 PM at one of the two entrance/exits of the Albertsons grocery store. Initial observations indicated that there was a consistent flow of pedestrian traffic in and out of the store. The longest wait time between potential participants was roughly two minutes. Additionally groups of potential participants exited the store at various times of the survey session. In a case like this, the first potential participant was asked to conduct the short survey and if they denied the survey then the next available person who was not engaged with their phone or children was asked. 27 surveys were conducted during this session over a period of an hour and a half. It must be noted that this Albertsons has a schedule of solicitors allowed to be at the entrance/exit of the store. Permission was granted between the times of 2:00-3:30 PM on this day from the store manager with the option to return on another day. A major observations was potential participants in the age range of 18-35 (the focus of this study) often declined the survey. Although it is difficult to know the exact age of those who declined, and it is estimated that there was a higher ratio of "young" adults declining versus middle-aged or older adults. While this study does not focus on the participation behaviors of young people with regard to public surveys, it acts as an anecdote to the overall participation levels of the youth in Atascadero. Those potential participants that may have been within the 18-35 age range was not recorded in this survey session. The data collected in this survey session was compiled with session 2 and is analyzed later in this section. 


\subsubsection{Survey Session 2 Observations - May 11, 2011 5:00-6:00 PM}

Survey session 2 was identical to session 1 except for the time of day. 5:00-6:00 PM was chosen in order to reach those who may have been at work until 5:00PM. This is assuming that the majority of residents ends their work day at 5:00 as well as conducts their grocery shopping after work. However, informal observations indicated that there was a higher ratio of young (18-35) adults who visited the grocery store at this time. As an amendment to the first survey session, frequency notes were taken of those potentially 18-35 who declined to take the survey. The total potential 18-35 year old residents that declined were 21 . This informal data is to be used as anecdotal information about the potential loss of survey data. Although this session was shorter than the first session, the majority of total surveys were collected during this time. 52 surveys were conducted with a maximum wait time between surveys of one minute. This data was collected and compiled with survey session 1 and is analyzed below.

\subsubsection{Compiled Survey Data}

Over the two survey sessions, 79 surveys were collected and analyzed for trends and/or correlation. Participants ranged in age from the youngest of 16 to the oldest of 80 with the average age of 45 . Specifically there were only 24 participants between the ages of 18-35 while the majority (70\%) of participants were older than 35 . There was a higher ratio of those potentially in the 18-35 age range that declined to be surveyed, perhaps leading to the skewed results. 


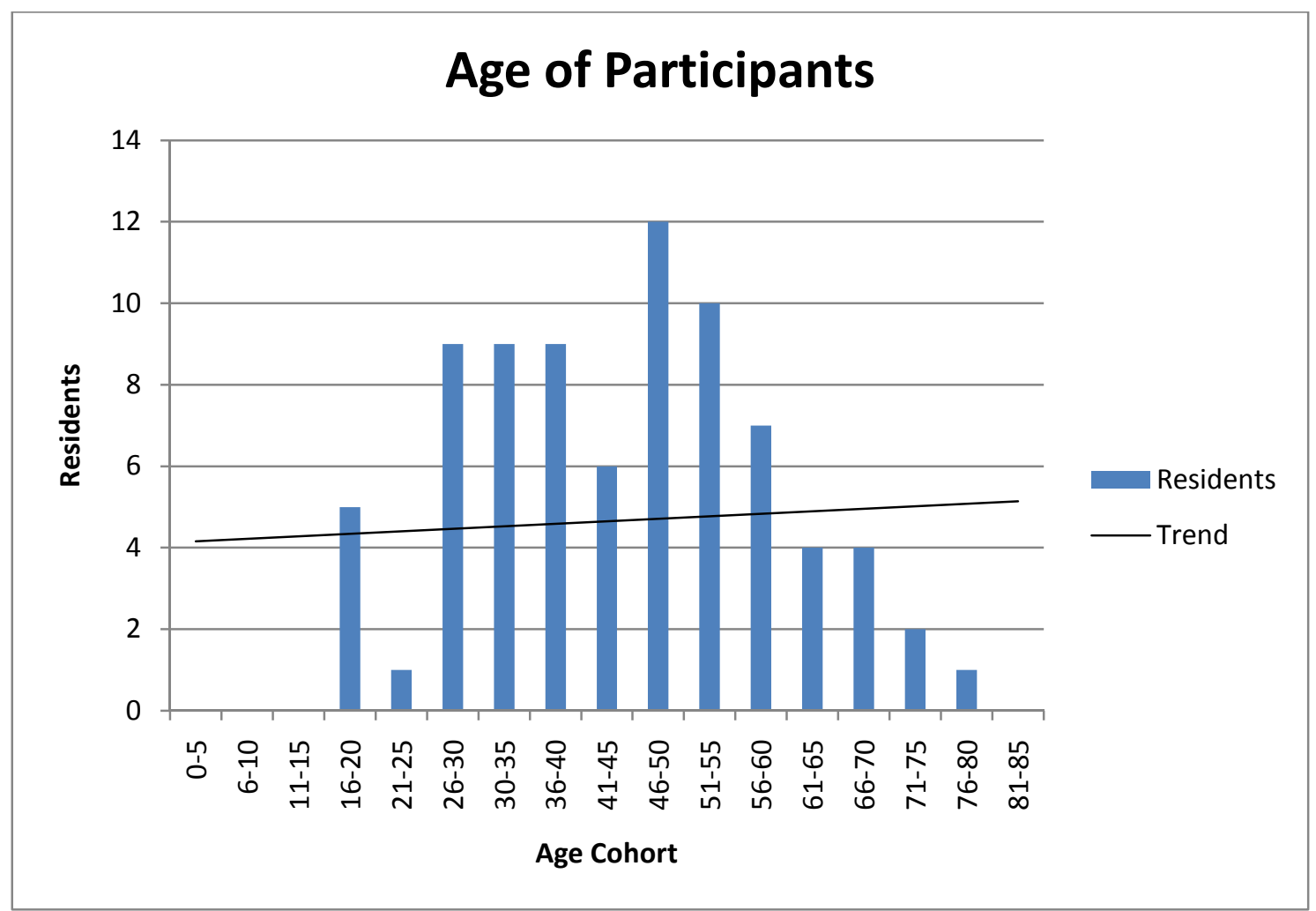

Figure 2 Age of Participants with Trend Line
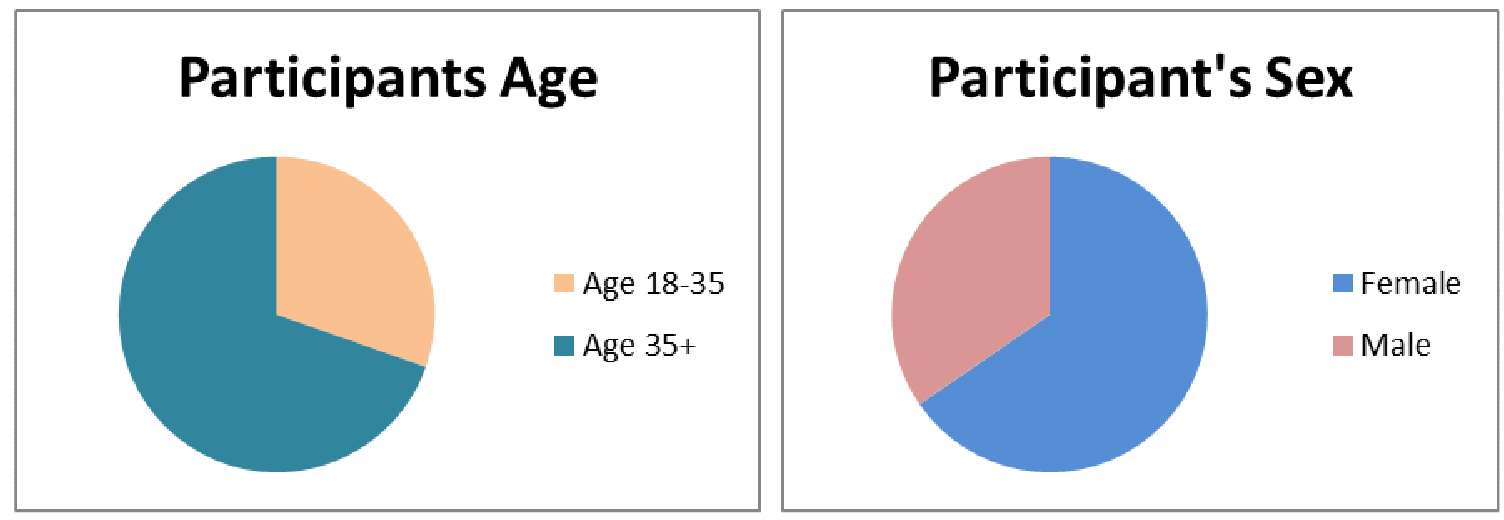

Figure 3 Comparison of Participants Age and Sex

Other notable demographic information is $65 \%$ of respondents were female with $34 \%$ male, although the sex of the participant had no impact on public participation.

Participants were initially asked if they have ever been to an Atascadero public hearing or public workshop for the city. Those that responded "yes" were asked two 
additional questions 1) How did you hear about the meeting, and 2) How often do you attend these meetings (per year)? Data indicates that $76 \%$ of participants have not attended a public meeting or a city run workshop. Therefore $24 \%$ of participants have been to or go to public meetings/workshops within Atascadero. When asked how they heard of the meeting $32 \%$ said they found out through word of mouth. This was in the form of either speaking to neighbors or heard about the issue at work and then deciding to attend the meeting. Sixteen percent of the respondents that attended the public meeting/workshop stated that they heard about the meeting because they were invited as a part of the meeting. An example of this is one participant was invited to receive an award at a meeting. Additionally, $16 \%$ heard of the meeting though a newspaper announcement. Of the top three methods of hearing about the meetings, only one (newspaper) is a typical/required method of outreach for the city. The top notification method is via word of mouth, which is an informal movement of information. It must be noted that Facebook was not mentioned as a method of notification. However, the Facebook page was still at its infancy during this data collection with 30 "fans", which would suggest why no one mentioned it as a form of notification. 


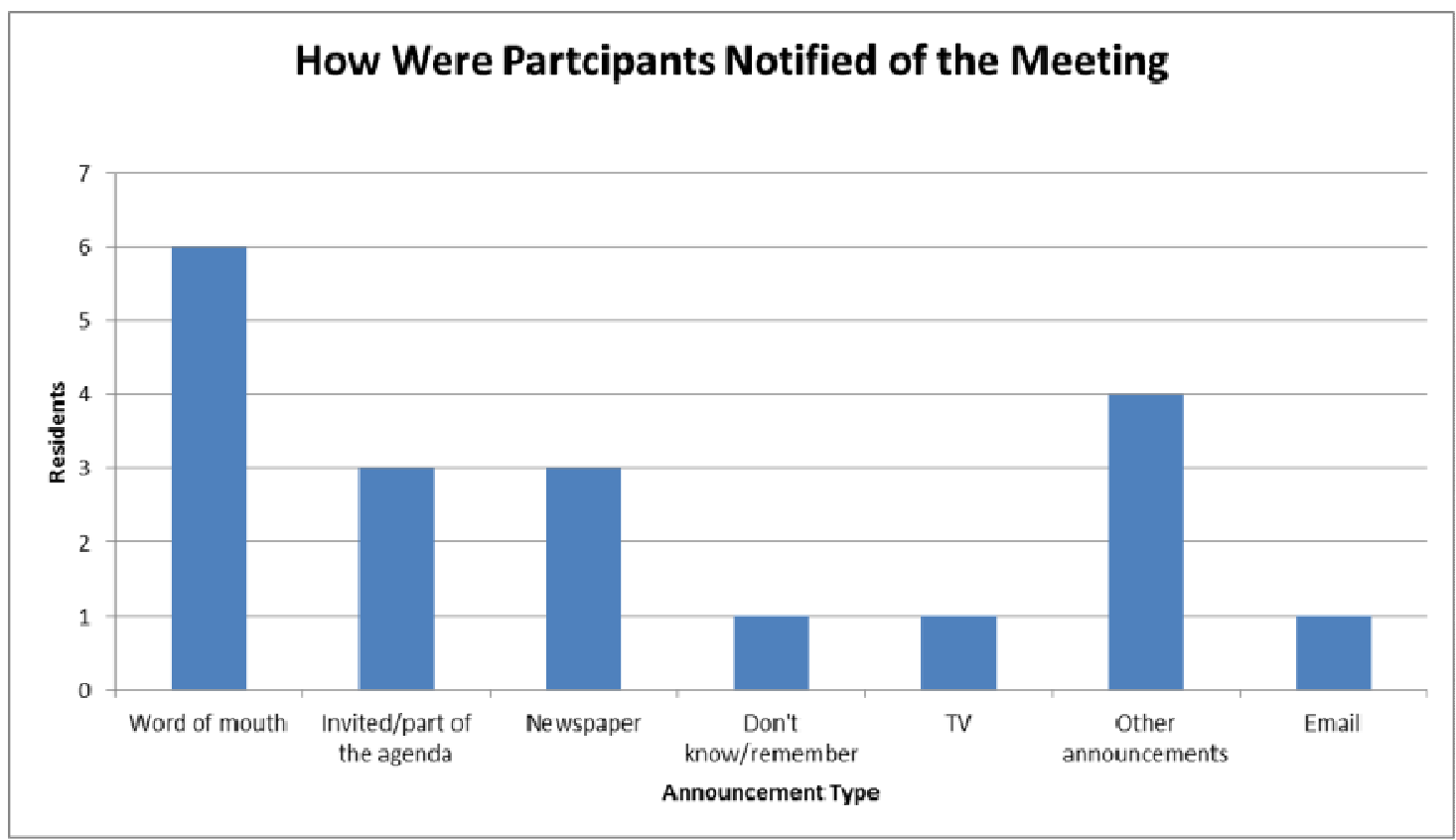

Figure 4 Notifications of Public Meetings

For those that do attend the meetings, frequency was asked to determine if they participate on a regular basis or if it is a rare occurrence. Fifty-three percent of those who have attended public meetings have only been to one meeting per year. Twenty-one percent attend or have attended meetings for specific agenda items and as such may have participated one time or multiple times but only dependent on the agenda item. This is opposed to those who attend meetings on a regular basis independent of the agenda item. As mentioned earlier, public participation is highly dependent on the agenda item at hand. For the case study of the April 5, 2011 Planning Commission meeting, the tree removal agenda item had only three participants (two of which were the applicant). Based on the data, those that attend public meetings do not go on a regular basis. 


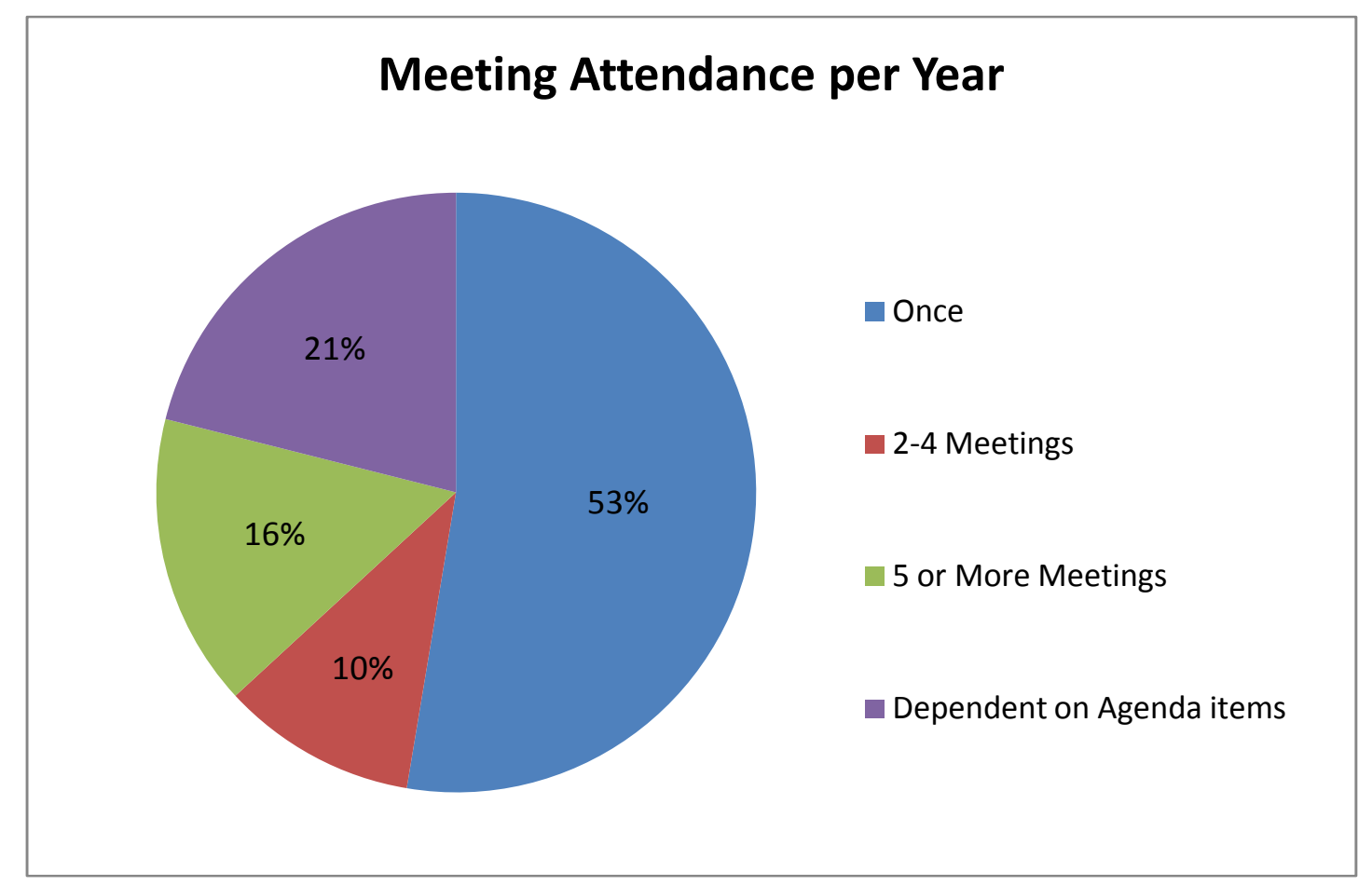

Figure 5 Attendance at Public Meetings

The majority of survey participants $(76 \%)$ did not or has not attended public hearings or workshops by the city. As a follow up question to respondents that indicated they don't attend public meetings/workshops, people were asked "what is the main reason for not attending". Twenty-five percent of the respondents said they have a time constraint which prevents them from going to a meeting. An additional 23\% said that they either don't know about the meetings or don't hear about them. Eighteen percent of respondents indicated that they didn't have a specific reason for going, they just haven't attended. Although difficult to determine if this is a true form of apathy or if because of the short nature of the survey, they did not have enough time to truly think of an answer. Fifteen percent of respondents said they were not interested in the meetings as their reason for not going. 


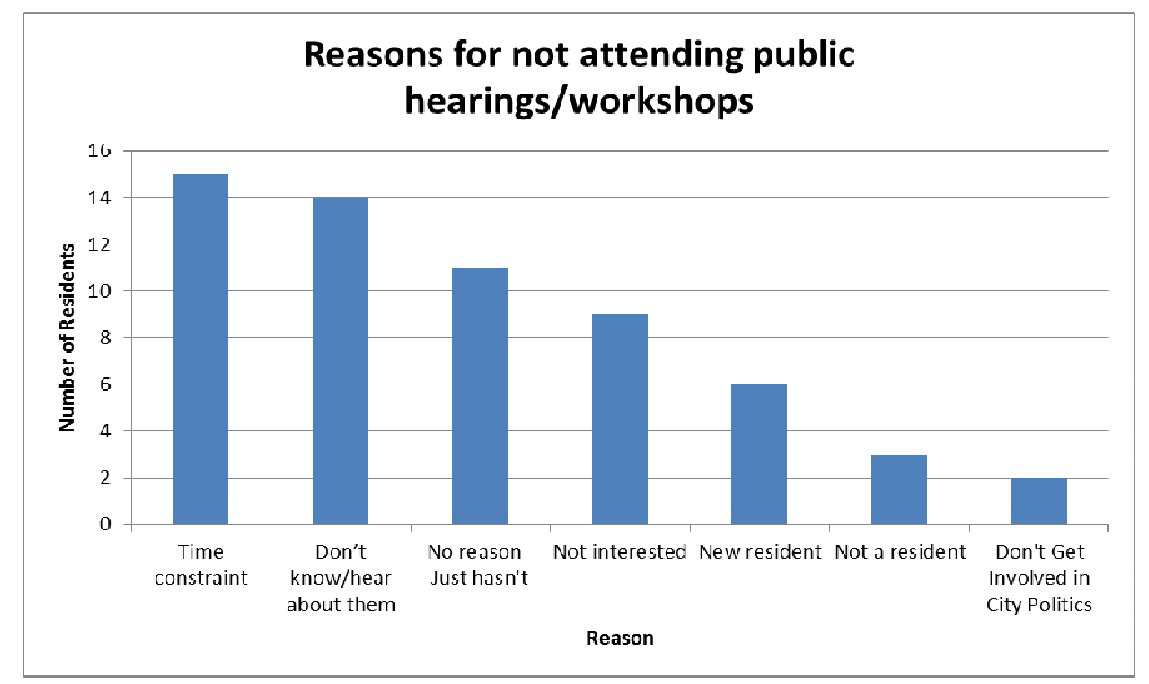

Figure 6 Reasons for Not Attending Public Workshops or Hearings

Based on the data, comparisons can be made with those who are on Facebook and those who attend public meetings. It can be seen in Figure 6 that the majority of respondents have not been to a public hearing/workshop while the majority is on Facebook. This provides a correlation between the two data sets that suggests there may be opportunity to use Facebook as an alternative method of outreach. Figure 7 compares the data of public hearing/workshop participants and people who are on Facebook.

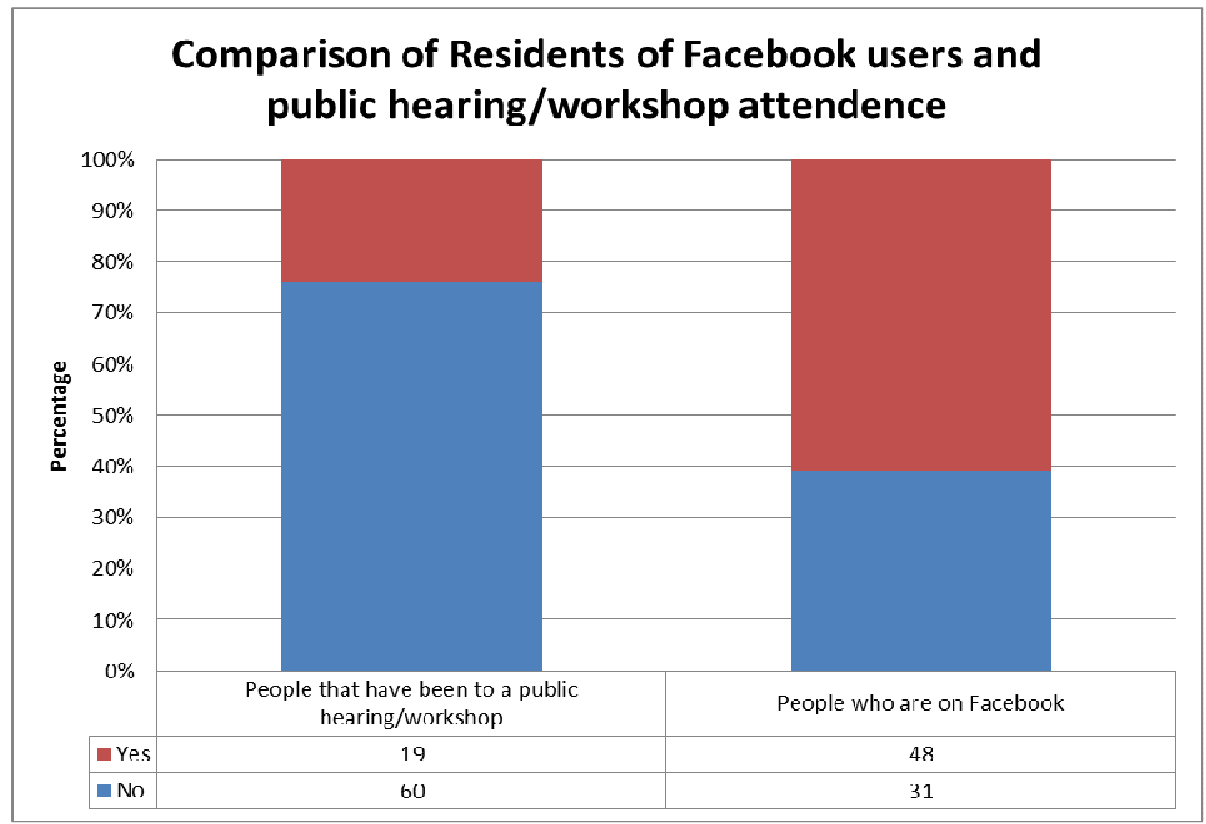

Figure 7 Comparison of Residents on Facebook users and Public Hearing/Workshop Attendance 
Data shows that participants on Facebook are comparatively younger than those not on Facebook. Of those on Facebook the cohort with the highest frequency of people was equally 26-30, 30-35, 36-40, 51-55 year olds. In comparison the highest frequency of people not of Facebook was equally 46-50 and 51-55 year olds. Figures 8 and 9 shows the age cohorts and trend lines for those who are Facebook users and those that are not Facebook users.

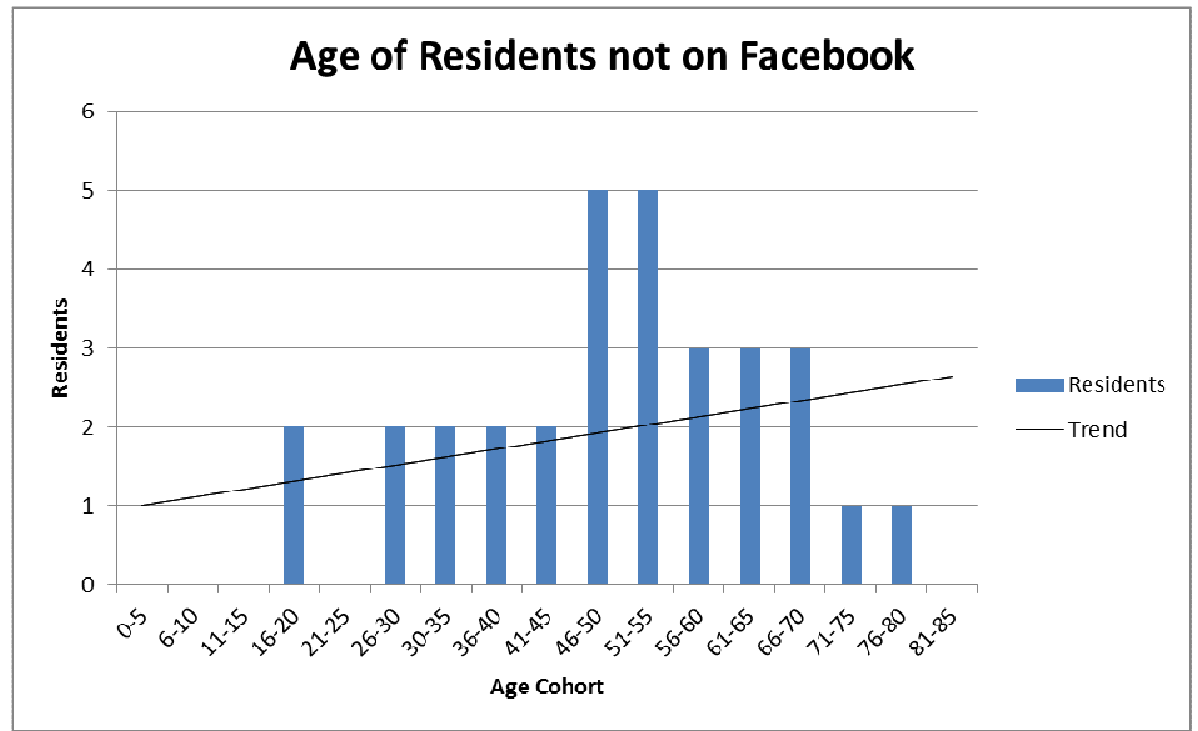

Figure 8 Non Facebook Users by Age

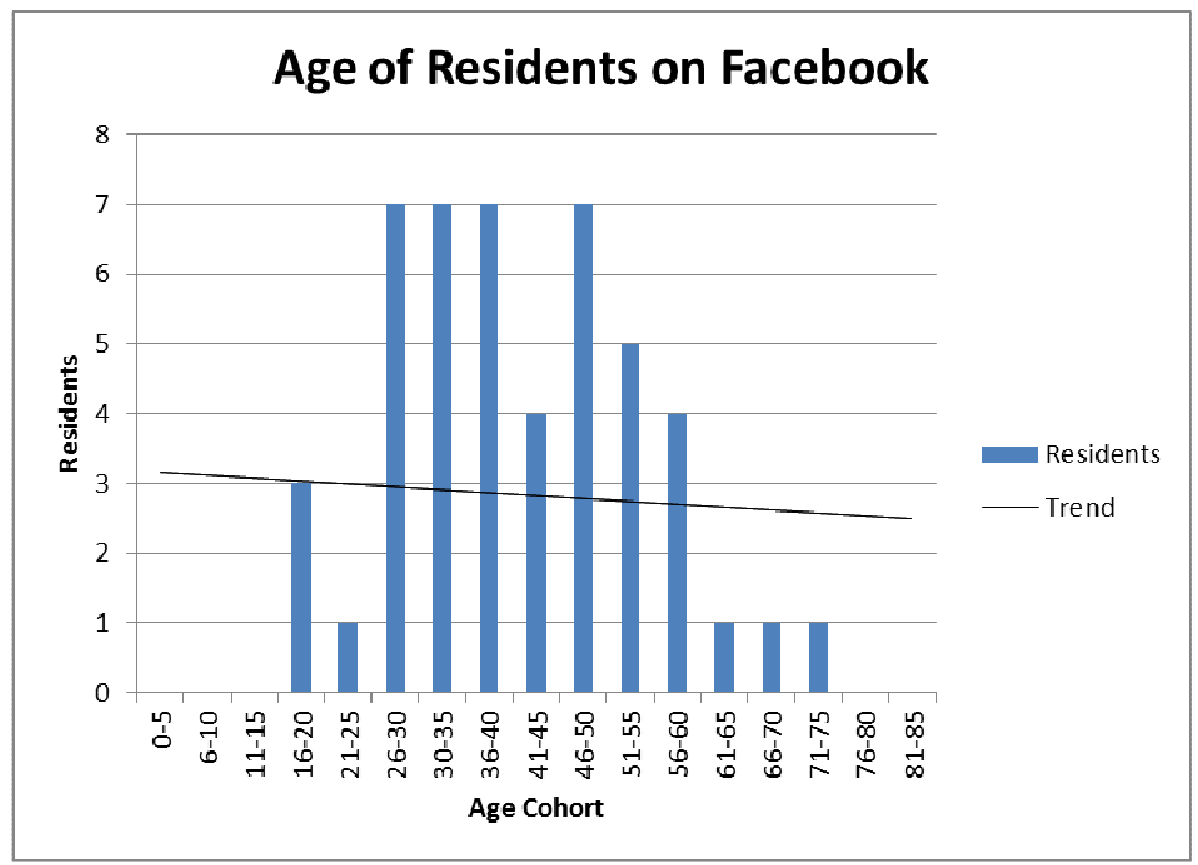

Figure 9 Facebook Users by Age 
Data shows a wider array of Facebook users' age than initially anticipated. Those considered "older" are now on Facebook, where the stigma of young people on the site has subsided as it's transformed into a way to connect with old friends, independent of age. However, as seen with the data, there is still a trend towards younger people on Facebook.

\subsection{Atascadero Facebook Page}

An official Facebook page was created for the City of Atascadero as a case study and to gather data on the various issues with creating and operating a page. The page was created through an adaption of Facebook's “Connecting to your Constituents with Facebook" which is essentially a best practice manual for general governmental organizations. In addition, the online govloop.com blog was used in conjunction with Facebook's manual to develop and implement the Atascadero Facebook Page.

\subsubsection{Page Development}

The process of developing the page based on the recommendations for both Facebook's manual and the govloop.com information allowed the page to be created in a few hours. Setting up a page requires a dedicated username and password that will essentially be the administrator for the page. The City of Atascadero was required to create a dedicated email address and password that only the administrator and Director of Planning knew. This process took 11 days to request and established a dedicated email address to be used by the administrator. This required a request from the Planning Department to Information Technology which should be taken into consideration for cities that might be on a timeline to launch their Facebook Page. Once the login information was established, the Facebook page could be created. However, the process 
requires the administrator to have a personal profile to establish a Page. After the administrator logged into Facebook there is a link to "Create a Page" at the bottom of the webpage. See the "Best Practice Manual" for more information on creating a city Page. The page required decision to be made by the City of Atascadero before making it available to the public. Facebook offers a lot of options for setting up a page, but the minimum information needed to launch the Atascadero Page is as follows:

- Profile Photo

- Wall and Commenting Policy

- Information Tab

- Terms of Use

- Photos

- Events

An understanding of these elements of the Facebook Page is important prior to developing the Page as each needs to be discussed in detail between the administrator and the department.

\subsubsection{Profile Photo}

Selecting a profile photo was important to the City of Atascadero because when searching for Pages within Facebook, photos are noticeable and assist users in quickly identifying the city. The use of the official city seal was discouraged as govloop.com indicates "it's suggested to not use the official agency seal but a compelling picture." (2011). It is assumed that government seals may not be recognizable when at a thumbnail size. In addition, using an official government seal may give the Page a "voice" of strict 
official business, whereas Facebook should be used as an informal "voice" in the same sense as a friend on Facebook. The photo selected for the City of Atascadero was the historic City Hall. This is a major icon in the City of Atascadero and is easily recognizable at any size on the webpage.

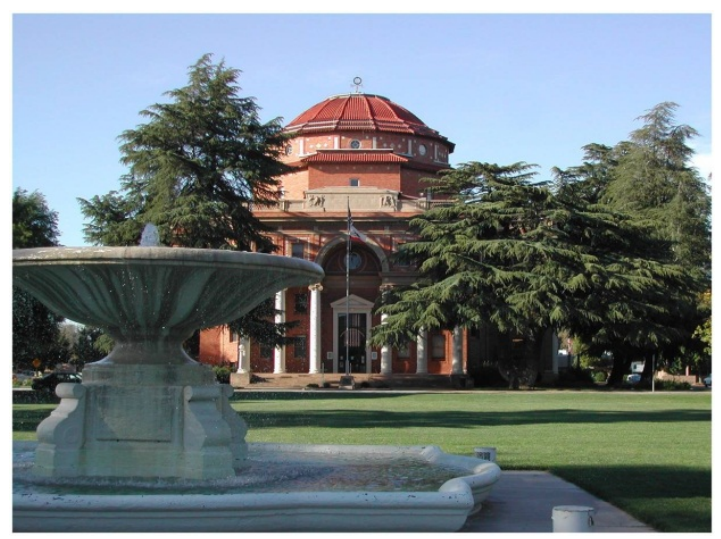

Figure 10 Profile Photo Selected for the Atascadero Facebook Page

\subsubsection{Wall and Commenting Policy}

The wall can be considered the main attraction of a Page, as it is where social interactions are public and media can be posted. The City of Atascadero made an important decision on restricting fan comments. These restrictions are in the form of limiting commenting, photo sharing, links, and video. The main issue was to determine if the city had the resources to monitor and moderate explicit comments, which would require staff to review the comment on a daily basis and delete if necessary. Commenting can be allowed or restricted in two distinct ways. The first allows fans of the page to directly post a comment, photo, video, or link on the Page. This option requires increased moderation as fans may leave inappropriate comments that are not relevant to the Page (i.e. spam or advertising for local services). The second option is to allow commenting on city posted updates such as status updates, photos, videos, or links. The distinction is that fans will be 
limited to commenting only on city produced update and thus reduce the amount of comments to moderate.

The decision for allowing comments is very important because the city is using a social networking site and as such inherently is a two-way communication portal. Limiting comments limits the two-way communication and essentially becomes a webpage (one way communication). The issue then is if the city wants to be open for true two way communication and be vulnerable to SPAM attacks or off topic posts by the public. Therefore the City of Atascadero chose to limit comments to city posted content only in the beginning. The city left the option to review the commenting policy at a later date with the option of opening comments to anyone wishing to post on the page.

\subsubsection{Information Tab}

The information tab is where users can find information about the city, including the address, date founded, hours of operation, description, mission statement, and contact information. This section also includes the option to create a username. It must be noted that creating a username for the Page requires at least 25 people who "like" the Page. Creating a username for the City of Atascadero was important to display the link to the page on printed material. Without a username, the address to access the page would be long and difficult to remember as seen here:

http://www.facebook.com/pages/City-of-Atascadero-Planning/175544509158142

Because the city wanted to include information of the Facebook Page on printed materials such as Planning Commission agendas, it was important to establish at least 25 friends to grant access to a custom username. Therefore the paradox was with advertising the page in order to get more friends in order to establish a username, but without a username it was difficult to establish more friends. The alternative to having a username 
to access the page was dependence on the search field. Potential fans of the page were given the page name "City of Atascadero Planning" to type in the search field and thus find the page that way. This was advertised at the front counter at the City of Atascadero with a small sign in addition to being posted on the Planning Department's board. Copies of the signage used can be found in Appendix D. Once there were over 25 fans of the page, the city had to decide what the official username would be. The initial username was to be "atascaderoplanning", however this did not become the final username.

Facebook has a strict policy of choosing one username without the option to edit it. Thus, once a username is created it cannot be changed. Additionally, usernames are available for both personal profiles and Facebook Pages. Facebook required the administrator to edit the username from the personal profile associated with the City of Atascadero and as such the username "atascaderoplanning" was synced with the personal profile rather than the official Page. Because Facebook does not allow administrators to edit the final username had to be different from "atascaderoplanning" and as such "planningatascadero" was chosen for the official username. Therefore users can simply go to:

www.facebook.com/planningatascadero

Once the username was established, the city could include it in printed materials such as the Planning Commission agenda for people to see and visit the page. Additionally the signage at the front counter was changed to include the new address as another means of advertising. 


\subsubsection{Terms of Use}

The "Terms of Use" section will most likely require consultation with the City Attorney.

This is section of the Page dictates the rules for public comment and posting media such as photos or videos. The City of Atascadero ensured the language was legal as well as direct:

This is an official Facebook page of the City of Atascadero. For more information about the City of Atascadero please visit Atascadero. This site is intended to serve as a mechanism for communication between the public and the Community Development Department to further our mission. Any comment submitted to this page and its list of fans may be considered a public record which is subject to disclosure pursuant to the California Public Records Act. Public information requests must be directed to the City Clerk's office.

Any comments posted to this page will be monitored daily and inappropriate content will be removed as soon as possible. Under the City of Atascadero Social Media Use Policy, the City reserves the right to remove inappropriate content, including, but not limited to, those items that have obscene language or sexual content, threaten or defame any person or organization, violate the legal ownership interest of another party, promote illegal activity and promote commercial services or products. The City disclaims any and all responsibility and liability for any materials that the City deems inappropriate for posting, which cannot be removed in an expeditious and otherwise timely manner. The City reserves the right to remove any fans that continuously violate this policy. Please enjoy this page and if you have any questions regarding the terms of use please email us at planningfb@atascadero.org. (Atascadero Facebook Page, 2011)

This language allows for everyone to comment, but rules are in place to remove explicit comment. It is important to note that the Facebook Page is an extension of any media publication from the city such as a press release.

Development of a "Terms of Use" section requires the use of FBML (Facebook Markup Language) which is a derivative of HTML (Hypertext Markup Language). The administrator had to create the Terms of Use section by adding it from the "Apps" option in profile editing section of Facebook. FBML allows for any content to be created as an additional section of the Page. This requires minimal HTML language knowledge in order to create page breaks and spacing. Advanced HTML/FBML knowledge provides 
for use of graphics and embedded links which is at the discretion of the administrator and/or the department running the page.

The only other potential restriction that the city had to be cautious about was the age restriction. Again, the city cannot restrict the public from viewing the page and as such must make it available to all ages. Facebook gives the Administrator the option to select restrictions for $13+, 17+, 18+, 19+$, and $21+$. The City of Atascadero ensured $13+($ the lowest age allowed) was selected so that anyone can view the page. Additionally Facebook allows for country restrictions, which the United States was selected.

\subsubsection{Photos}

Photos are one of the media types that can be posted on Facebook and as such the City of Atascadero wanted to include a wide range of photos from existing to proposed projects. Photos provide succinct information to be shared with fans of the page. However, choosing appropriate photos was an important challenge for the city. It was decided to create photo albums of which the primary album is named "Concept to Construction". This album has been created for two separate projects within the city. The premise of this photo album is to show fans the submitted elevation plans for a project and then take photos of the project throughout the stages of construction. This provides fans with information about projects in their area and gives them a single source to see development on projects. However, no parameters have been set by the city to determine which projects will be given a spotlight in the photo section of the Page.

\subsubsection{Events}

The events section of the Page allows for the city to create event notifications that can be monitored though rsvp (for fans of the page only). Creating an event is relatively simple, 
where Facebook asks for the date and time of the event, the type of event, the location, and a section for more information with an option to add a photo. The city used this option to create a Planning Commission Meeting event with additional information that links back to the city's website. Although fans can rsvp to the event, none of them did. The events section is only an effective tool if fans are aware of the new event which is why it should be shared on the wall for all to see.

\subsubsection{Additional Tools}

Additional tools/applications were used beyond the recommended minimum as mentioned above. Facebook provides for a multitude of applications that can be added to the Page in addition to FMBL created sections.

\subsubsection{PDF/Doc Formats}

This application allows the city/Administrator to upload PDF files to a section of the Page. Any PDF file can be uploaded and so the administrator used this tool to upload copies of the various Commissions' agenda. Multiple files can be uploaded with specific titles to display in order to keep track of the files available to view. In addition the application can be renamed, which the City of Atascadero named "Agenda/Documents". This is an optional tool that currently has one Design Review Committee Agenda available.

\subsubsection{Discussion}

The discussion section provides for a topic specific area for fans to talk. Like a typical online forum, discussion items are listed and fans can view each topic and give their personal opinions. However, the City of Atascadero has made this section available, but no topics have been added to the selection. This tool has potential to allow fans to 
participate on items of interest, such as new projects, General Plan updates, and other planning related topics. This section could be used as a secondary tool to workshops where public comment/input is a vital role to the development of plans or policy. This area could allow for fans to leave their comment if they could not attend the workshop or public hearing. However, like the commenting section on the wall, comments in the discussion area will need to be moderated.

\subsubsection{Twitter}

This section was added by the administrator as a portal to the city's Twitter account. Cross platform promotion or linking is relatively common and is an easy way to disperse information thought the various social media methods. The Twitter section provides a view of all the Tweets that the city has posted of which may or may not have the same information posted on the city's Facebook wall.

\subsubsection{Page Insight}

Facebook provides a tool to administrators to monitor activity on the page. This data is split between "Users" and "Interactions" which can be viewed on various timelines. It must be noted that some data was not recorded until the page had over 25 fans. The user information indicates that April 25, 2011 was when the page exceeded 25 fans although the page was established on February 25, 2011. The first data set displays active users for daily, weekly, and monthly interactions from fans. It can be seen that active users peaked for the month of April and has been on a steady decline during the month of May. There is no obvious reason for this peak and then decline other than word of mouth prompted the increase in fans over the period of April. 


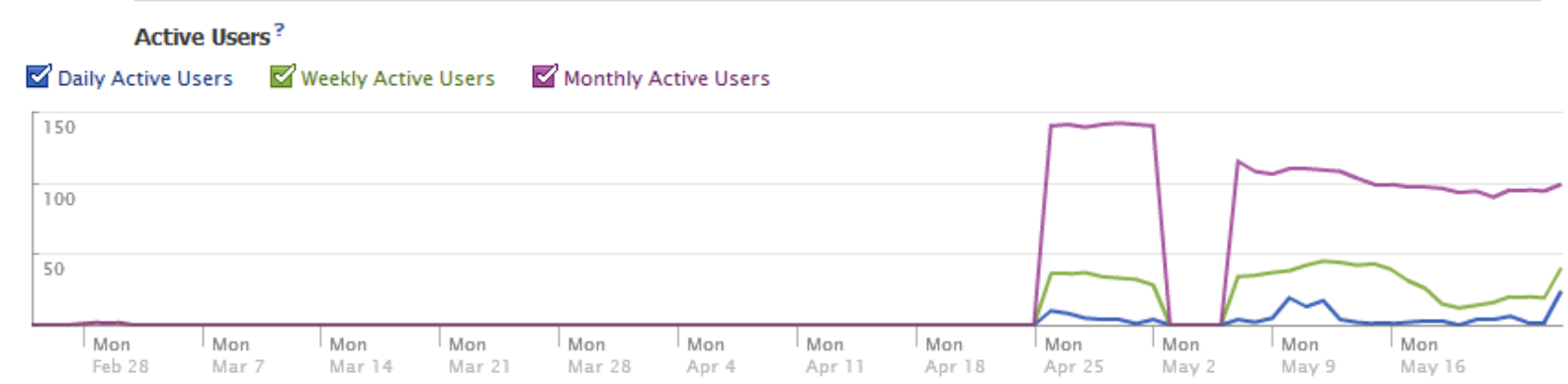

Figure 11 Active Users - 2/25/11 - 5/26/11

The next data set presents the daily active user breakdown. This information indicates what users interacted with while on the page. The highest level of interaction was simply view posts authored by the city. Unique Page Views accounts for individuals who visit the page and disregards how many times they click on the page per day, it still only calculates that person visiting the page once. "Liked a Post" data is show for those people who have read an update and have clicked the "like" button next to the post. Comments on posts (the only comments permitted) have not produced recordable data until recently where more and more fans are commenting. Two spikes can be seen May $10^{\text {th }}$ and May $12^{\text {th }}$ which corresponds to the city posting about Colony Square - a relatively new project with a state of the art movie theater and associated retail locations. The posts indicate that there are new tenants moving into the vacant retail spaces near the theater. The spike on May $26^{\text {th }}$ correlates with a post about the Eagle Ranch project which is a contentious project for the city and as such garnished two comments from users.

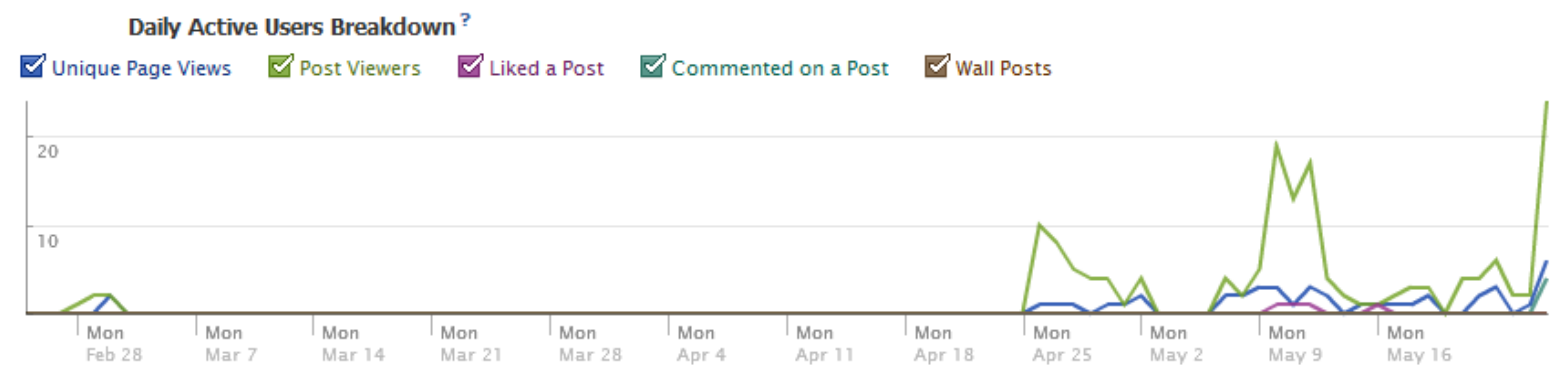

Figure 12 Daily Active Users Breakdown - 2/25/11 - 5/26/11 
Another important data set is the "New Likes" graph that can either show how many people liked per day, or over a period of time. The daily like graph shows that there is a maximum of one like per day prior to May $26^{\text {th }}$. After May $26^{\text {th }}$ there is one day with two people "liking" the page. Addition this graph shows who "unliked" the page which accounts for one user on May $25^{\text {th }}$. When looking at the Total likes graph, it can be seen that there is a steady rise of likes since April $25^{\text {th }}$ until May $26^{\text {th }}$. It must be noted that throughout the graphs there was an error that prevented data from May $3^{\text {rd }}$ to May $6^{\text {th }}$ to show. This is seen on all graphs and the origin of the error is unknown.

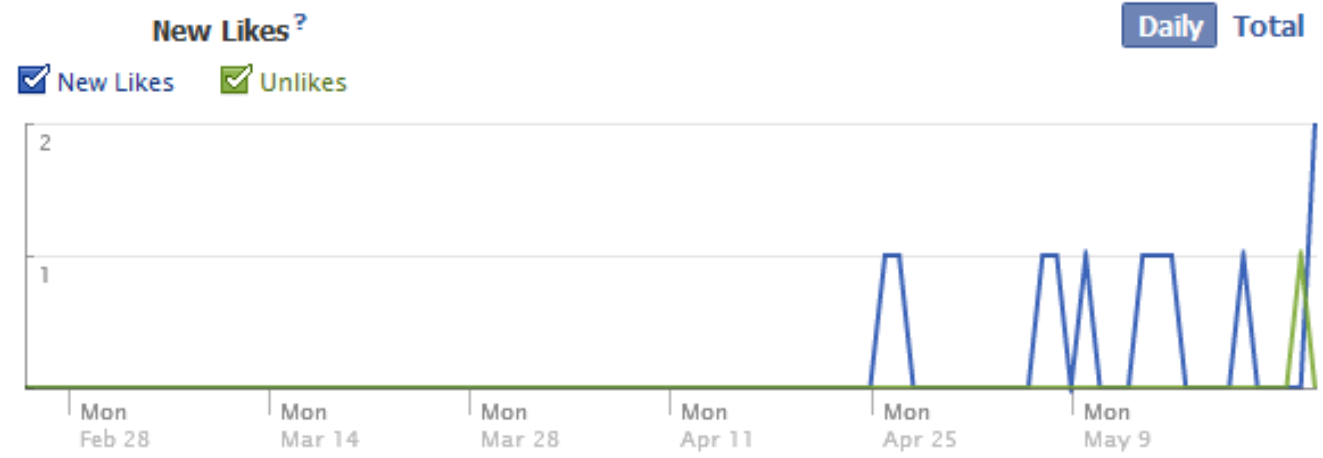

Figure 13 Daily Likes - 2/25/11 - 5/26/11

Total Likes?

Daily Total

Total Likes $\square$ Total Unsubscribers

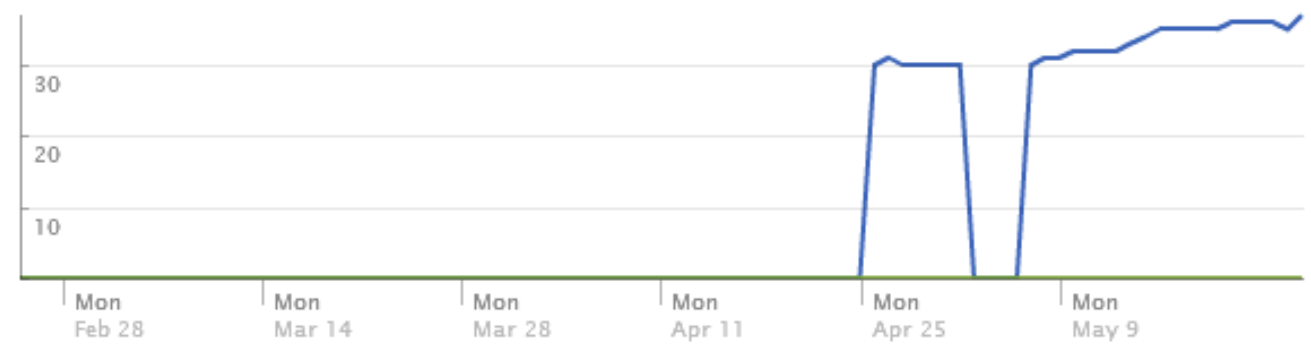

Figure 14 Total Likes - 2/25/11 - 5/26/11

Because users register their age, location, and sex, Facebook can produce accurate demographics of those visiting the page. The following graph (Figure 15) shows a breakdown of age cohorts and sex based on the 38 fans of the Page. It can be seen that the 
age cohort 18-24 is not present. This is because no users fall into this cohort and so the graph omits the information rather than leave it blank. It can also be seen that the majority of users are females 25-35 years old. Males account for $14 \%$ of fans in this same age cohort. Although information for the cohorts 45-54 and 55+ should not be ignored. These two cohorts combined account for $37.7 \%$ of users while the $18-34$ cohort accounts for $38.7 \%$.

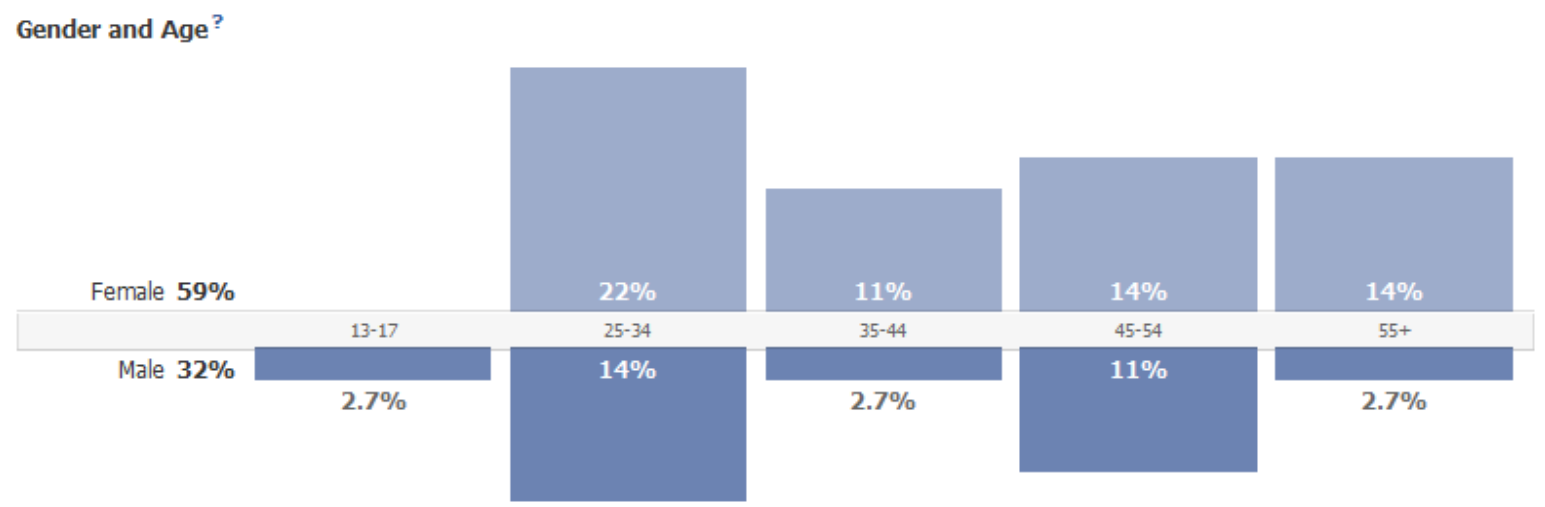

Figure 15 Gender and Age Demographics

The data for activity and page views indicates that a majority of users view the wall, with a total of 152 views. The following graph represents page views which account for total page hits as well as unique page views which accounts for registered Facebook users viewing the page. 


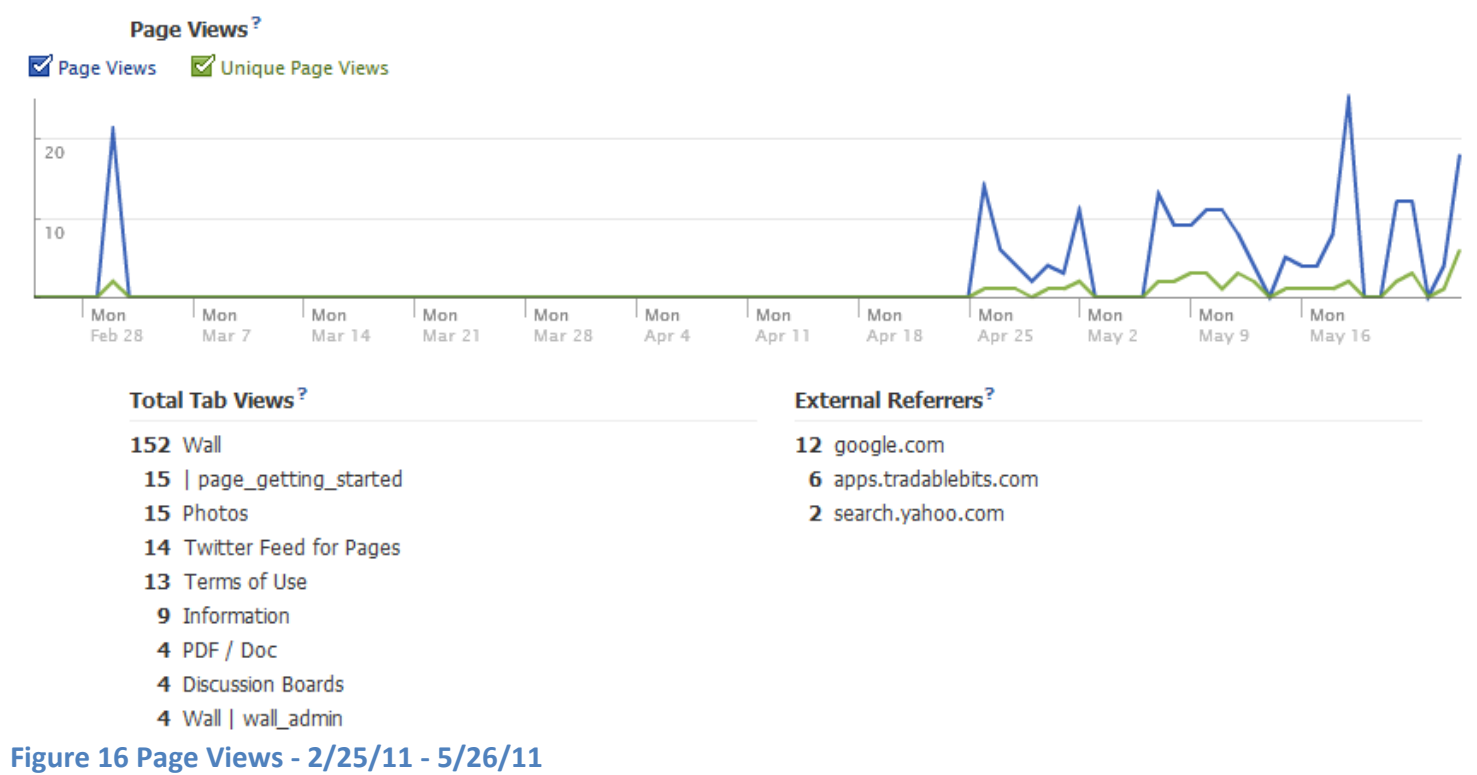

The above graph shows that although there is a low level of unique page views, there are many page hits per day. This can be attributed to non-Facebook users visiting the site via the badge located on the city's website. Essentially anyone can visit the page and not be a registered user; however they are limited to access features of the page as well as post comments.

\subsubsection{Promotion}

The city's page required marketing in order to gain fans/viewers, however this was limited any kind of free promotion available. The simplest way of promoting the page was through the use of "like badges". These are icons of various sizes that can be embedded in websites such as the City of Atascadero website. The like badge is the most common form of marketing a Page through another webpage. In addition, the icon used for the "like" badge can be on printed material as it is easily recognizable and associated with Facebook. The Administrator had to work with the Information Technology department at the city to establish an area to place the badges and determine what type of badge to use. It is important to establish a plan with the IT department to determine when 
the city's webpage will need to be updated and if there will be a dedicated social media page. The City of Atascadero's webpage features a right hand navigation bar that appears on each page of the city's website. Therefore the "find us on Facebook" badge is located on this navigation bar so that it is available independent of what page the user is visiting.

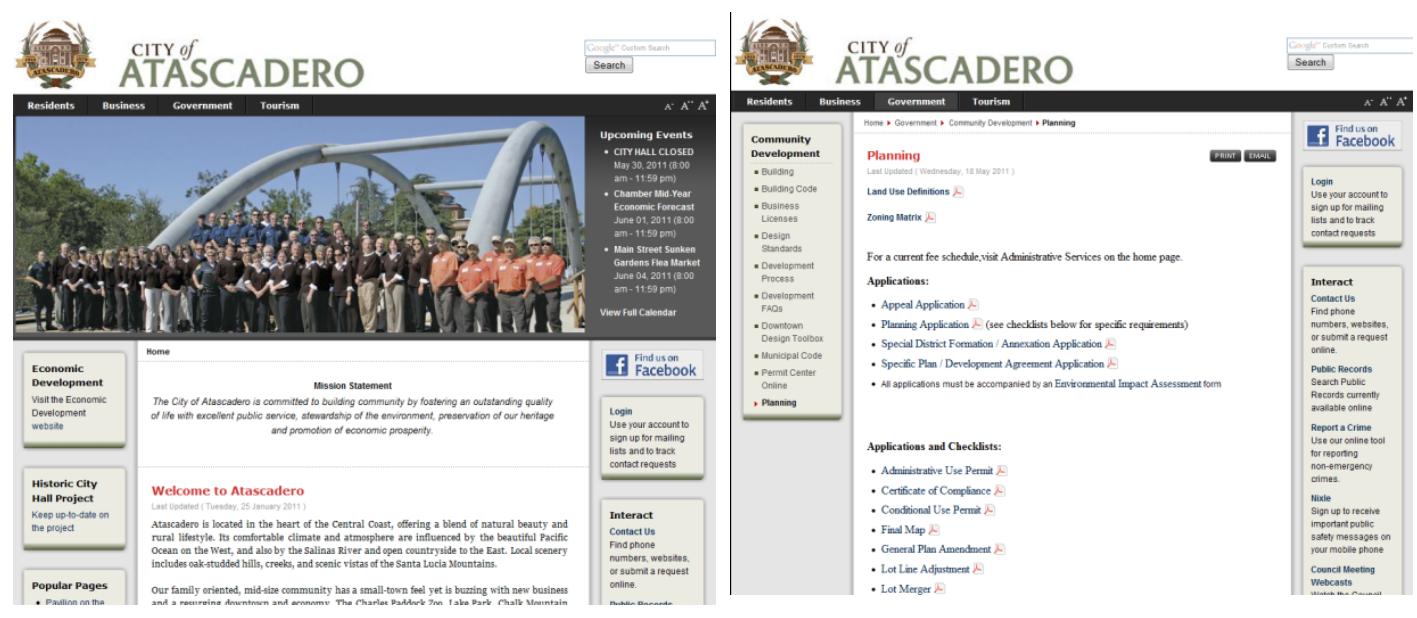

Figure 17 The Facebook Badge is Located on Every Page of the City's Website

Once this badge is clicked, it takes the user to a direct webpage dedicated for social media. Currently the page features three Facebook pages; Atascadero Community Services Department (Parks \& Recreation), City of Atascadero Planning, and Atascadero Fire Disaster Response Trailer. Each of these Facebook pages is run by the city and located in a central area on the website. The city decided to use a specific type of badge, the "activity feed" not only provides a link directly to the Facebook Page, but there is a window that provides real time updates of the Page without the user leaving the website. 


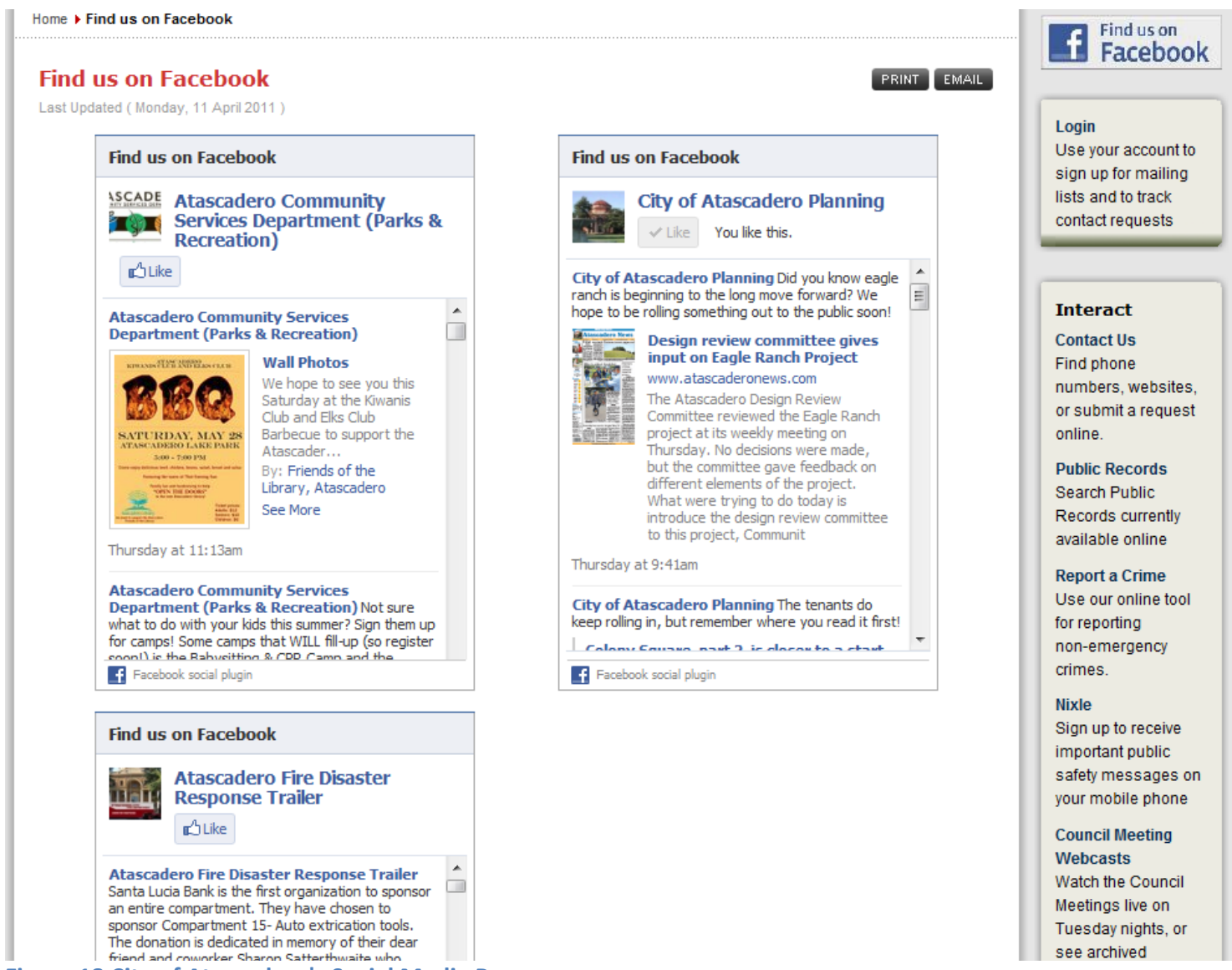

Figure 18 City of Atascadero's Social Media Page

\subsection{City Interviews}

\subsubsection{Background}

A total of 102 cities were contacted via email to various cities across the contiguous

United States. The interviews included six questions for the appropriate person to answer.

Questions were sent only after a contact was established with the city. This is to say that

an initial email explaining the study and the area of interest was sent to either 1) a general city email address 2) the Information Technology department 3) the Communications department 4) the Planning department or 4) the City Mayor or Clerk department. The email explained the study and asked to be put in touch with the most appropriate person to answer them. Of the 102 cities, 28 cities responded and requested the interview and 13 cities responded to the interview questions giving a $12.7 \%$ response rate. cities chosen for 
interviews were based on accessibility to their Facebook page. In other words, the city's website was accessed and if there was no obvious Facebook or social media link on their homepage, then it was determined they didn't have a page. Additionally Facebook could have been used as a search tool for city Pages, but there was no guarantee that it was the official page. Whereas any link on the official city webpage would link to an official Facebook page. As mentioned previously, 28 cities responded to the initial email to send them the questions, those that did not respond were sent a follow up email. Of those that were sent a follow up email, none replied with their answers.

The Facebook Pages were reviewed for authenticity to ensure 1) the Page was under "government organization" type of page and not a personal profile, or a company, and 2) the Page was either for the city as a whole or specifically the planning department (not the Mayor's Page). It was found Wikipedia.com generates generic Pages for cities and some cities linked their city website to the Wikipedia page (which is not run by the city). cities that did this were not counted or emailed the interview questions.

\subsubsection{Primary Goal of Using Facebook}

Many cities use social media beyond Facebook, such as Twitter, YouTube, and blogs. As a goal these cities wanted to create a form of outreach that 1) Informed a broad spectrum of residents that may not otherwise participate in city related activities and 2) Take advantage of the benefits of social media at no cost to the city. As such, $85 \%$ of respondents indicated that the page was developed to reach the community and disperse information to them. Additionally, $15 \%$ of respondents directly mentioned the goal of reaching a younger demographic. Social media is different from a website or an email blast (both of which are methods for communicating with the community), however 
Facebook allows for the city to see which residents are following the page as well as generate demographic information.

\subsubsection{Deciding to use Facebook}

Respondents were asked who was responsible for deciding to move forward with the use of social media. This information provided feedback on generally which sector of the city is in charge of the development of the Page. There were a variety of responses and as such no one department is consistently responsible for creating or championing the creation of the Page. It should also be noted that there is not a typical standard naming convention for departments. Therefore, a city with a Municipal Information office might share the same duties as another city's' Communications office. However, $15 \%$ of respondents indicated that the Mayor's office was responsible, while another $15 \%$ did not know who was responsible. The majority, $54 \%$, of responses suggested that the Community Relations, Public Information, Municipal Information and Public Affairs departments/offices were responsible for the idea to expand with social media. These departments/offices were responsible to weighting the benefits of a Facebook page and the potential risks associated with it. Many cities felt that a Facebook page and/or Twitter account would be useful in emergency situations for the dispersal of information to a large number or residents in a short amount of time. It should be noted that of all the respondents, only one was a Planner with the Cincinnati Planning department.

Additionally respondents indicated that there were multiple Facebook pages for both departments and policies. For example, cities had pages for the Parks and Recreation department, Police, Fire, and Public Works department, while other cities had Pages for Specific Plans and in the case of Cincinnati, a 2010 Census Page. Therefore the use of 
Facebook goes beyond the typical department run Page and can be used for specific policies or plans.

\subsubsection{Operation of the Page}

Cities operated the page in generally the same method. This was with a single administrator that was responsible for the daily operations while multiple administrators were available as "backup" if the primary administrator was unavailable to create content or update. However some cities have a single contact or single department where all of the content is sent and reviews and then posted by. For example with the City of Tampa, Florida the Public Affairs office ran the page originally whereby requesting all content be submitted to them for review and then it would be posted. However, because of some departments having a high volume of content to update, the policy has changed to allow those specific departments to post content without the need to go through the Public Affairs office. $54 \%$ of respondents indicated that there was a single administrator although multiple administrators had access and would only post content as backup to the primary administrator. One city explained that there was just one login account that was shared among staff members. Additionally there were no reports of security concerns from this city. Fifteen percent of respondents mentioned that there were no security concerns due to the fact that only executive level employees are allowed to post on behalf of the city. Essentially those who have authorization to create press releases or speak to the media are the ones allowed to post of Facebook. Therefore there are no security concerns as of yet as each city had taken the measures to ensure trustworthy staff is responsible for the page. 


\subsubsection{Internal Protocol for Operating the Page}

Respondents were asked if there was any specific protocol or policy that staff needed to follow in order to operate the page. Of those surveyed, $38 \%$ had no such policy or protocol and instead used discretion to what to post and how to respond to residents on the Page. Although Pocatello, Idaho doesn't have a policy, the city plans to adopt a protocol when the social media/web use policy is updated. Therefore $62 \%$ of respondents had a policy for operating the Facebook Page that was either created for the specific Page or adapted from an existing policy. The policies range in detail but ultimately they cover the city's responsibility for responding to comments or other forms of feedback while also setting regulations for the removal of explicit content and content not related to the city's Page (Advertisements, Spam, and off-topic comments). Additionally one city's policy indicated that while the policy does not restrict personal use of social media outside of the office, it encouraged the use of good judgment. This is to say that as a city employee, items discussed on the personal pages must not be representative of the city and a disclaimer is encouraged with the language of "The postings on this site are my own and do not represent the city's positions, strategies or opinions." cities also have to be aware of any Open Records law that will require any posts to be subject to a request for records. Facebook provides the ability to download all the information shared from the Page within the Account Settings. Although some cities have taken daily screen shots of the content to save for any open records requests. It also should be noted that there is not a standardized policy that cities use, instead either the department/office creates a specific policy for Facebook, or a specific Policy for social media in general or there is no policy and the city is to use discretion when operating the page. 


\subsubsection{Issues and Concerns with the Page}

Due to the nature of Facebook and social media in general, it was necessary to determine if there were any issues with developing, implementing, and operating the Page. Because Facebook allows for two-way communication between the city and residents, as well as all communication is open for others to view on the wall, it was necessary to understand the major issues at hand. However, 54\% of respondents indicated that there were no major issues with operating the page. Although, those same respondents had slight concerns with the production of content. The concern with content was in two forms, first the type of content and ensuring that it represents the department (if department run) or represents all departments equally (with regard to general city Facebook Pages). The second concern was flow of content, where some cities would have a lot of information within a short period of time which meant that there were times when the city had little or no information to share. Updating the site often is necessary to keep the interest of fans and Pages that are not updated often (more than three times per week) often are looked at as stagnating and lose fans. Beyond creating content, commenting was a concern by many cities whereby they allowed the public to comment on the city Page but were responsible for removing explicit comments, advertising, other types of spam comments, and any off-topic (non-city related items). Because the page required a moderator that was responsible for removing comments on a regular basis, the issue of staff-hours became apparent. If there is not a dedicated position for maintaining the page, then staff must update it when there is free time. Thirty-one percent of respondents indicated that staff-time was a potential concern for keeping the page updated as well as monitored for comments and other types of media. Another concern was access to Facebook from city 
computers, where many cities deny access to social media sites from the workplace. An example is Cincinnati, Ohio where initial access to Facebook was denied and as such any updates to the city Page needed to be from home on personal time. This was recently changed but is an often overlooked area for cities wanting to build and run a page from the workplace.

\subsubsection{Obtaining Goals}

Each city indicated goals for developing the Facebook Page and therefore was asked if they achieved these goals. Of the cities interviewed, $85 \%$ responded "yes" to achieving their goals. It is important to set goals for Facebook and social media in general as there are no standardize measures for determining the effectiveness of a Facebook Page. For the most part, goals were obtained through increased fans, where $46 \%$ responded with a specific number of fans achieved. The City of North Charleston, South Carolina achieved the goals set out and mentioned that it was attained without any cost to the city (except for a one-time $\$ 20$ fee for additional hard drive space). cities responded with emphasis on reaching a broader demographic through the use of Facebook when compared to the typical outreach methods. Additionally some cities feel that they achieved their goals but have not achieved the full potential of Facebook as a tool. 


\section{Discussion}

Analysis of the Atascadero city Facebook Page case study, the general surveys, and the interviews with cities indicate current trends and opportunities. In general, residents of Atascadero did not attend public meetings, where only $24 \%$ attended meetings and of that $24 \%, 15 \%$ were invited to attend the meeting. In addition of the people who attended public meetings, only $26 \%$ were notified through the typical outreach methods (newspaper, TV, and/or email). Although many people did not attend meetings, the majority of respondents indicated that they were on Facebook $(61 \%)$ which suggests an opportunity for them to connect with the city virtually by becoming a fan of the Page. The city's Facebook page provided insight into the behavior of fans and suggested that ample time is needed to gain popularity. After the launch of the page, promotion was needed in order to gain a fan base. Due to budget constraints, only free marketing was available in the form of word-of-mouth as well as signage within City Hall. There is opportunity to gain a larger fan base if there is a budget for Facebook advertising. Essentially Facebook advertising will allow the city to alert Facebook users registered in Atascadero of the new page. This method is rarely used as seen in the city interviews, where taking advantage of the free service was priority for many cities. Additionally the cities specified their initial goal was to use Facebook as an additional tool to reach a broader audience, and in some cases, reach a younger generation.

The findings show that there is a general trend towards the use of Facebook independent of age. The use of Facebook as a participatory tool is still a relatively new concept and as such the following factors must be identified to determine the opportunities and constraints of the service: 
- Outreach

- Content

- Security

- Department/Office

- Facebook Users

- Time-Cost Benefits

- Integration with Existing Technology

The following sections review these factors in extended detail based on the Atascadero city Facebook case study and the public and city interviews.

\subsection{Outreach}

With the launch of the Atascadero city Facebook page came an assumption that like many things online, it would go viral. This is to say that word-of-mouth would lead to a gradual increase of fans where in reality the page did not increase the fan base until roughly a month after launch. Atascadero has a population of 28,310 (US Census Bureau, 2010) and as such may not garnish the same amount of fans as cities with a population of 50,000 or more. The word-of-mouth method of outreach is highly dependent on users of Facebook to intentionally tell friends and family about the page so that others become fans. The signage within City Hall may have led people to the page, but then they may not have felt that becoming a fan was necessary. Outreach of the page is difficult for cities as seen with the city interviews. Many cities across the United States don't have the staff, let alone the budget, to operate and advertise the page. So, free promotion is necessary in most cases. The public surveys indicated that a majority of respondents were on Facebook, independent of their age. This majority has the potential to view the 
Facebook page and become a fan. Other methods of outreach are necessary to inform as many Facebook users as possible of the page. The existing forms of outreach are links within the city webpage, signage at the public counter in City Hall, and the address printed on agendas (such as the Planning Commission). Additional forms of outreach can be in the form of Facebook advertising, flyers, announcements at Planning Commission or City Council meetings, Advertising on TV during or before televised Commission meetings, and/or a link on the signature lines of city staff.

Facebook advertising allows administrators of Pages to create an ad that will be promoted on the right hand side of the target audiences' Facebook profile under a section called "Sponsored". The ad can have a photo and up to 134 characters of text in addition to a title. This ad can then be targeted to a specific location (city of Atascadero), and age range, sex, interests, and specific fans (i.e. targeted to fans of the Atascadero Recreation department). Facebook is unique because users input their demographics and interests when creating a profile. Thus Facebook is extremely precise in advertising to specific people. However this feature is not free and requires the administrator to enter the maximum amount of money to spend on advertising and Facebook will automatically create an ad campaign based on that number. Using Facebook ads has the potential to increase the fan base of the city Page but without a dedicated budget, it is not a viable option.

Flyers can be created at a relatively low cost and inserted into water bills or made available at public workshops, the public counter, or any event that the city hosts or sponsors. Flyers offer a form of promotion to potential fans but may not target specific Facebook users as well as the Facebook ad system works. However, creating flyers for 
people to take with them and/or show their friends and family is an augments method of word-of-mouth. Getting information out about the page in any form possible is vital for increasing the fan base.

An announcement at Planning Commission or City Council meetings is free promotion and requires little or no resources to produce. The administrator of the Facebook Page would be responsible for getting the information to the Planning Commission or City Council and encouraging them to announce the launch of the page and how to access the page. This can also be done in the form of advertising on televised Commission or Council meetings. Typically there are time slots for displaying messages either before the meeting or during a break. Information can be displayed about the launch of the page with the accompanying Facebook icon and username.

Normally city staff has a signature line that is at the end of an email that includes their name, position, address, and phone number. Another line can be added to this signature to include information about the launch of the Facebook page. This information can be a single line with the Facebook icon and the hyperlink to the page. This is essentially free advertising but will only reach a small audience that may or may not be Facebook users.

Outreach and promotion is essential for increasing the fan base of the city Page. An increased fan base will allow the city to communicate with residents on various city related issues and receive feedback that may not have been possible without Facebook.

\subsection{Content}

Although having a large fan base is important, it is the decision to become a fan that requires additional research and insight. However it can be hypothesized that one of the 
reasons for a resident to become a fan of a city is because of the content. Content provides incentives for Facebook users to become a fan of a page in order to receive information that they may not otherwise receive. Fans most likely are subscribed to a number of different Pages that range in topics, therefore an incentive must be there to draw viewership to the city's Page. Content is one of the biggest concerns with city's as seen in the findings. This concern is in two parts, 1) the type of content to post and 2) the frequency of posting content. Content must be interesting and act as an incentive for fans to come to the Page frequently. Facebook measures feedback per post as well as total impressions. A Post with a lot of impressions essentially means that it was viewed either on the city's wall or on a fan's wall who shared the post. In addition to impressions, there are statistics for feedback based on the amount of views. The City of Atascadero received both high impression and feedback rates for posts about "Concept to Construction" where new projects are highlighted with photographs. Additionally a post about the Eagle Ranch design review committee input gathered only 116 impressions while providing $2.59 \%$ feedback rate. This post included a link to a newspaper article on the Eagle Ranch project and gathered two comments in addition to two likes for one of the comments. Updating on the Atascadero Page is done on a weekly basis. Although there is no formal or scientific study on the optimal number of times to post to a Page, it is suggested that updates be done on a regular basis. Based on the results from the study of random California Facebook pages (see Appendix E), cities updated at least once a week with some updating more than once a week. Content on Facebook is like content on any other type of website or blog, and therefore must be updated on a regular basis to capture the attention of the fans. 


\subsection{Security}

Of the cities interviewed, 54\% reported that there was a single administrator in charge of operating the page on a regular basis. However some cities explained that there were multiple administrators who are used as backup in case the primary administrator is unavailable. Ideally a single administrator ensures the Page will be consistent with the types of updates as well as the "voice" of the page. Because Facebook is a social networking tool, each Page or organization will have a "voice" as if the page were a friend in a social network. This voice can be open and friendly though the choice of language within the posts/updates, as well as the types of photos and media posted. Alternatively, the voice can be formal and serious which is not recommended for this social media setting. It is not recommended because Facebook is an informal place for friends to meet and network. This can be seen with the jargon used via Facebook such as "liking" a post/Page, "poking" profiles, and "tagging" people, places or things.

Additionally the section of a Page for correspondence and sharing is called the "wall". More formal social networking sites exist, such as LinkedIn.com, a professional networking site dedicated to connecting career-minded people across the world. The administrator is responsible for promoting the Page and giving it a voice that people can connect with. Therefore security concerns arise when the administrator may not have the city's best interest in mind. If the city delegates all administrator rights to a single person who does not share the login information with his/her superiors then they will have complete power over the page. If, for example, the administrator is let go or resigns from the job with the city, there are potential consequences of a disgruntled employee. Creating multiple administrators is therefore encouraged as backup to the primary 
administrator for this very reason. As mentioned before, appointing one individual to update the page ensures continuity and reduced confusion in the case of multiple administrators updating with the same information at the same time. One city that was interviewed explained that there was one login account that was shared among staff members so that they could update the page autonomously and not be required to send their updates to a single administrator for approval. Again, this indicated a security concern as multiple people could post under a single name. In the case of misinformation being posted, or again, a disgruntled employee, the city would have no control over recovering the page. The City of North Charleston, South Carolina ensured security through only appointing executive level staff that had the same rights to deliver a press release or speak to the media. Although this ensures a higher level of security, it may present new constraints. The major constraint with updating the Facebook Page is the staff-hours needed to operate, moderate, and maintain the Page. By appointing an executive level staff administrator rights, there may be an issue of staff-time devoted to updating the page while also maintaining the other executive level responsibilities of the employee. Tampa, Florida is an example of one solution to this constraint, where one administrator was appointed, and staff would send any and all updates to this single administrator. Thereby reducing security risks by creating a filter for content as well as lower level staff hours devoted to updating the page. However, as with the case with Tampa, departments began to create high volumes of information/updates and as such caused strain on the administrator responsible for filtering content. Eventually departments producing high volumes of information have direct access to post on the Page. Therefore cities should review three things prior to launching a page to determine 
the type of administrator rights. 1) How much content will be posted per week, 2) Where is the content coming from (multiple departments or a single person?), and 3) How many staff-hours can be devoted to the page? To review, if there is a high flow of content to update at least once per day, then it is suggested that there be a primary administrator with multiple administrators as backup to the primary. If there are multiple departments producing content for a single page, then each department should have an administrator to post. In addition each department should follow an internal protocol to determine posting criteria and the voice of the page. And finally, if budget restricts the staff-hours dedicated to updating the page, then again, multiple administrators should be given the rights to post. This is opposed to a single administrator that would need to review and update content, and thus dedicates a majority of his/her time to Facebook. It is recommended that a single administrator login not be shared with staff in order to ensure tracking of who is posting what on behalf of the city.

An additional constraint to internal security is the security established by city's Information Technology department. Many cities restrict the use of Facebook from a city computer, such as the cities of Atascadero and Cincinnati. Facebook was established as a site for people to maintain their personal profiles and networks, and only recently has the company expanded to included organizations and businesses. As such, many companies and organizations such as city departments restrict the use of Facebook on company time. Therefore when creating a page, the administrator must work with the department responsible for blocking websites, which in many cases will be the Information Technology department. An additional constraint is creating a dedicated administrator login profile. In order to create a Facebook Page, administrators must register a personal 
profile page and then create an organization Page. The login information requires an email address and a password, which is suggested that the email address be consistent with other city addresses. An example would be facebook@ city.com or facebookadmin@city.com. These addresses are generic and provide a single login standard for administrators using Facebook. cities that wish to launch a page under a certain timeline must remember to consult with the IT department as there may be delays in both creating a username (email address) as well as unblocking the site to specific administrators.

\subsection{Department/Office}

This study focused on how planning departments can use Facebook as an additional tool to reach residents and engage in public participation. However, as seen with the interviews, much of the city run Facebook pages are operated by different departments and staff positions. These include the Public Information Office, Office of the Mayor, Management Analysts, Communications department, Community Outreach and Participation, Information Technology Department, Public Affairs, Community Relations, and Planning. Although a variety of departments may run the main city's page for their respective city, there are specific Facebook Pages for departments. However, despite the focus of the Page (citywide, specific department, or a plan/policy) administrators will follow the same steps required to set up a Page. The main constraint when multiple departments are involved with a Page is providing content in a consistent flow and that the administrator is able to filter and post information efficiently. For department specific Pages, there is opportunity for a steady flow of content with a single administrator responsible for the updates. It should also be noted that there is no formal 
distinction between the content in a citywide Facebook page versus a planning specific page. However review of the citywide pages suggests intradepartmental updates, such as updates from public works on a specific issue, or transportation with a road reopening. There may be overlap between the content on a planning specific Page and the citywide Page, but again there are no specific limitations to what can be posted on either type of page.

\subsection{Facebook Users}

The general surveys suggest that there is a trend towards younger (18-35) adults on Facebook (Figure 9) when compared to those not on Facebook (Figure 8). However, results indicated there were a large number of adults older than 35 years old who used Facebook. As the service becomes popular as a networking site, people independent of their age will join. Facebook has over 500 million active users worldwide that is increasing on a daily basis (Facebook, 2011). An average user is connected to 80 community pages, groups and events (Facebook, 2011). With the average age of respondents for the public survey at 45 years old data suggests that there is an increase in 35 years old and older on Facebook. As an anecdote, it must be noted that there was a disproportionate amount of potential participants that denied taking the survey. Although it is impossible to know how old the potential participants were, an educated guess would put them between the ages of 18 and 35 . Frequency notations were taken on the second survey session of potential participants that denied taking the survey. It was determined that 21 potential 18-35 year old participants denied taking the survey. Therefore the demographic of Facebook users based on the survey may be skewed towards an older age. 


\subsection{Time-Cost Benefits}

As mentioned previously, the major concern with operating the Facebook Page was the staff-hours needed to update. Of the cities interviewed, none determined the staff-hours to be a major issue; instead it was a concern for cities to be aware of. Updating the page required the administrator to gather information, either in the form of a website link, media upload, or a simple status update. It may be time consuming for staff to gather content to post in order to maintain regular updates per day or per week. Staff that requires input from other departments may find it frustrating to receive information at various times of the day or week that may not coincide with an established updating schedule. Portland, Oregon uses an editorial calendar as a way to monitor updates and provide them consistently rather than all at once or no updates for long periods of time. Again, there are no formal or scientific studies to suggest the optimal schedule for updating a page, it is suggested that three times a week is considered regular. If cities use an editorial calendar to create and release content on a regular basis, then staff time can be divided among many days rather than all at once on a single day.

\subsection{Integration Within the Department}

Facebook is intuitive and designed to be as easy as possible to create, operate, and share content. Although there are many options available to administrators, the basic functions of a page are relatively easy to use. The use of Facebook does not require any special software skills or intimate knowledge of the service. Instructions are provided throughout the creation of a page and a status bar determines how complete a page is (i.e. if a profile photo is missing, or if no contact information has been entered). Users are only required to know how to access the page from any computer and/or mobile device. In addition 
Facebook can be used with other web applications such as Twitter. Although not reviewed in this study, Twitter is the second most widely used by the cities interviewed. Twitter provides information succinctly with a limit of 140 characters per post with unlimited posts. Facebook can integrate Twitter as seen with the Atascadero Page, where any Twitter updates are routed to a section within the Page. Cross promotion and use of multiple web technologies is an effective way to promote the city and gain Facebook fans and/or Twitter followers.

As previously mentioned, the IT department should be consulted to integrate the different functions of Facebook within the city webpage. There are multiple badges and buttons that connect users from the city website to the Facebook page. The most popular is the "like" button which can be embedded in the city website or specifically related to city news entries. Atascadero chose to embed a status update badge that allows users to view the updates on Facebook within a window in the city webpage (see figure 17). Of the cities interviewed, none responded with any issues with integrating it into existing technology or any additional staff hours needed to train on the use of Facebook. The relative ease and at no cost provides incentives for cities to integrate this tool into existing methods of outreach in order to better serve their communities. 


\section{Conclusions and Implications for Planners}

The world of social media is in its infancy with regard to it being used as a tool for planning practitioners. However it has been useful for creating an additional dimension of public participation for various cities as the findings indicate. Analysis of the Atascadero case study in conjunction with the interviews with cities that utilize a Facebook Page indicate opportunities to expand the reach of public engagement and participation through social media. The information presented in this study has been compiled into a Best Practice Manual at the end of this section and can be used by planners and/or cities that wish to adopt social media.

The manual provides a step-by-step guide that reviews the various options presented when creating a Facebook page. Explanations are given for each option presented in the profile settings in order to better guide planners to create a page that conforms to their own terms of use. The findings indicated various ways of using the Page as an outreach tool and thus planners/cities using the manual may develop their own use for the page. This manual is intended to be a general guide for creating a Facebook page, but it must be noted that some sections of the manual may be applied to other social media networks.

Although this study focused on Facebook as the main social networking service, it is suggested that Cites expand their use of social media as a whole. Such programs exist that will allow administrators to update multiple social media services at once and thus make use of time efficiently. The top two other social networking services used by cities are Twitter and YouTube. Each has their own opportunities and constraints, where Twitter provides succinct updates that can reach multiple users at once. YouTube 
provides a video service that allows cities to showcase different projects, features, and/or people. The purpose behind social media in general is to provide a short message to a mass of users in real time and allow the same users to respond. The ability to respond is what Deshler \& Sock explain as "Genuine Participation" and provides a way for the public to be heard by their local government. This is beneficial in many ways, but mainly there is a sense of transparency and trust between government and residents which are a main component of the public participation process (Portland Development Commission, 2007 p.77). There is a plethora of existing social networking services and assumed that new and improved services will be developed in the future. However, it is assumed that social media services will maintain fundamental functions, and that planning practitioners should be aware of these basic functions.

\subsection{Review of Research Questions}

This study intended to explore the use of social media, specifically Facebook, by cities as a public participation and outreach tool targeted at youth. This section is dedicated to reviewing the questions and drawing conclusions based on the findings.

1) How can Facebook be used as an efficient means of community outreach/participation?

Facebook is a relatively new tool for many of the cities interviewed, yet all have obtained the goals they set out initially. cities were able to launch and operate the Page with little resources as it is a free service and updating/operating the page is relatively simple and quick. The Pages can customized to include such things as Planning Commission agenda's in PDF format, or include surveys to understand residents wants/needs on particular issues, and photos can be used as an informal 
visual preference survey. Typical forms of outreach and/or participation activities can be translated in the realm of Facebook and as such provide an efficient means of outreach/participation.

2) Is there a difference between age groups as age relates to the use of Facebook for public participation?

Although this study focused on outreach and public participation from young adults, 18-35, findings show that Facebook is used by a wider variety of age cohorts and isn't necessarily limited to young people. However, the public surveys indicate that there is a trend towards younger people on Facebook; this does not produce conclusive results to suggest that young adults use the service exclusively. Of the cities interviewed, many suggested that the initial goal of creating a Page was to reach a "younger generation" or "residents who may not otherwise participate in city events". However, none of the cities fully focused on gaining young adult participation. Instead gaining young adult participation was a by-product of creating the page. This is seen through the Insight (Facebook's analytics) on the page, where the Atascadero case study indicated the largest age cohort was within the 18-35 range. Therefore, instead of focusing on using Facebook as a tool to reach young adults, it should be used to reach those who may not contribute through traditional methods of public participation. This may include young adults, but does not limit or dictate the use of Facebook as a tool specifically for this age cohort.

3) What are the ways in which users access Facebook to be informed about city activities? 
cities cannot ensure that the public will view updates posted on Facebook and rely on providing interesting content on a regular basis to pique fans' interest. The Atascadero case study provided data of the most viewed and most commented content. Hot topic items return a higher feedback rating than other less interesting (to fans) content. This may seem obvious, but although Facebook is a two-way form of communication it is difficult to know what people think of items posted if they do not respond. Responses from the public are open for anyone (fans and non-fans of the page) to see, and therefore people may not respond to items as openly as they would in a public workshop setting.

Users can access the page from any computer or mobile device with an internet connection. Furthermore when they access the page they can see if the city has posted event details and respond to content posted at any time of the day. Facebook provides a means for residents to interact with their government in an informal way and as such creates an opportunity for additional public participation. This is not to say that Facebook/social media will eventually replace traditional methods of public participation, yet it will augment these methods.

4) Can city residents who do not attend public meetings be informed via Facebook? In a word, yes. Some of the cities interviewed indicated that status updates were derived from press releases, one city in Oregon posted a questionnaire about a particular plan that was being developed. There are various opportunities for cities to use Facebook to inform residents and many cities already do this. The Atascadero case study provided information to the public on new projects and encouraged any and all feedback. Additionally the City of Atascadero has the 
ability to post PDF copies of agendas/minutes or any other city related documentation. As always, the public is encouraged to participate in the traditional workshop or public hearing setting, but generally Facebook can fill information gaps for residents who cannot make it to such an event.

5) What are the practices of those cities that use Facebook as an outreach and public participation tool?

cities use Facebook in a variety of ways, however many follow fundamental practices that allow them to be open and transparent. Most of the cities interviewed created multiple administrators with a primary administrator dedicated to updating and maintain the Page. The additional administrators were used as a backup to the primary in the case that they were not able to post or moderate the Page. The amount of time to update and/or maintain the page also varied from city to city. However the act of posting a status update is relatively quick. If an administrator uses official press releases as the basis for updates then it is a matter of condensing the information into a status update. Other time commitments are finding and posting links from websites or posting officially on other Facebook Pages. Perhaps the most time consuming is creating and posting media such as photos. However photos of a project or used with permission from a website (i.e. a developer of a large project in the city) can be time consuming as the photos must be uploaded and optionally "tagged" with a caption. If a city updates the page often (three or more times per day) then it is likely this will require a larger commitment from the administrator. Multiple administrators can 
alleviate the time dedicated by one staff member and provide a more efficient process for running the page.

\subsection{Future Research}

This study began to uncover an emerging trend of how cities use social media as a tool for public participation and outreach. Additional research is needed in order to fully understand the implications for planning practitioners. The design, development and implementation of this study set the basis for further research where a case study was developed, cities were interviewed, and the public answered behavioral questions. The Atascadero case study did not produce as many fans as initially anticipated and thus did not provide ideal data collection. This is, of course when comparing it to other cities that have thousands of fans and thus provide more data. However, with this being said, the case study set the ground work for interested cities who may be hesitant to embark on creating a page.

In addition, future research should look at and compare other forms of software such as CommunityViz and Community Planit that are dedicated to virtual public participation. A comparison would provide information on the efficiency of using social media as a platform versus dedicated software. Another aspect is place based software, this is where programs like CommunityViz require participants to be online within a set location (i.e. computers set up at a workshop). On the other hand Community Planit brings "together the interactivity of social networks and the incentives of online games, Community PlanIt transforms participatory planning into a fun, engaging activity for all ages." (Gordon, 2010). Further research would be able to draw parallels and perhaps inform those using social media as a planning tool to the future of public participation. 


\subsection{Required Resources for Planning Practitioners}

The collected data provided an awareness of the resources needed to create and maintain a Facebook Page. This section provides information of the resources required to create and maintain a page.

Administrators - In order to create a page, the city or department must select administrators. It is recommended that more than one administrator be created in case of the primary administrator is unavailable. Administrators are responsible for creating content as well as moderating comments from fans. This will require dedicated staffhours for maintaining the page and will vary from department to department. Administrators will be required to log in to Facebook at most, once per day, and at least once per week. Because administrators are responsible for moderating commenting, a daily browse of comments is recommended so that the Page does not contain explicit comments over a long period of time. The main reason for removing such comments in a timely manner will show fans that the department is dedicated to maintaining the page which in turn adds to the legitimacy of the page as a portal for the city.

Comments - Administrators are responsible for posting comments and updates to the Facebook Page and as such will require time and other resources to complete. Dependent on the size of the city/department running the page, posting comment may be on a daily basis or at least on a weekly basis. Larger cities may have more news to share which will allow the administrator to post a status update or a link to a press release or local news article. Posting an brief update can be completed in under five minutes as it is merely entering text. However, posting links can take additional time for the administrator as he/she will need to search online for the news article, webpage, blog, or other type of 
media and ensure the article is relevant to the city and fans, then copy and paste the link into the status bar. This may require more staff time devoted to researching articles or websites unless there is a protocol for sharing links, such as only posting links to the city webpage for news releases and updates. This type of protocol will shorten the amount of time needed to update and post links. The final type of content available to post is photographs and video media. cities wishing to update with this type of media on a regular basis will need to allocate more staff time to maintaining the Facebook Page. Photos/videos must be captured either from the primary source, or selected from an online source. There may be the need to edit photos or video such as cropping parts of the image out or editing the length of the video. This will not only require more staff time, but may require a higher level of photo/video editing software. Once this media is ready, it must be uploaded from a workstation to the Facebook page, and may require additional time if uploading large or multiple photos/videos.

The minimum requirements for a department deciding to use Facebook are as follows:

- Administrators must dedicate time to monitor and maintain the page at a minimum of once per week. This will ensure fans know the page is regularly updated and that any explicit comments, spam, or advertisements posted by fans will be removed in a timely manner.

- Administrators must also allocate time to research news articles or other web-based content to post on the Page. This may require searching the internet for news articles on a particular project and ensuring the source is legitimate which will require time from the administrator. 
- Content must be posted at least once a week to provide fans with current information and to avoid a stagnating page. Content can be in the form of a simple text based status update to an edited photograph or video.

- If the department decides to engage in the Facebook advertising option, the administrator(s) will be responsible for maintaining the marketing schedule and processing the fees required to post adverts. This may require additional meetings with department managers and will require a dedicated budget.

In short, fans will benefit from a page that is updated on a regular basis with interesting content. Both large and small cities can utilize Facebook as a tool for outreach/education, and fans will get as much out of the Page as the department puts into the Page. If these minimum requirements are met, then cities or departments should pursue creating a Facebook Page and following the Best Practices Manual provided in the next section. However, if it is found that these requirements cannot be met, then perhaps other forms of social media can provide a better platform for outreach and education. An example of another social media tool is Twitter. Administrators have just 140 characters to express their message of which can contain links to webpages or media (such as photo and video albums). Twitter has "followers" instead of "fan", but essentially are the same. Followers must find and select "follow" to receive updates from the administrator. This form of social media may be better for cities with low resources as it is a short messaging service that focuses on getting the message across to a select group with minimal maintenance. 


\subsection{Best Practices Manual for Planning Practitioners}

The following section is a dedicated best practices manual for planning practitioners. This manual is intended to be used by planners/cities that wish to include Facebook as a part of their outreach, education, and public participation. Although this manual is focused specifically on planning/planners, it can be easily adapted to be used by cities in general, where the main difference is the content that will be posted. It is assumed that there is a base level of understanding with regard to Facebook and social media as a whole and therefore this manual should be used by those confident in such software. The information presented is compiled from the data in this study as well as Facebook's own Best Practice Manual for Government (also known as "Connecting to your Constituents with Facebook). The manual is intended to provide information on how to create, launch, and maintain a Page.

\section{Five Key Recommendations}

This section provides the top five recommendations for cities or departments interested in creating a Facebook Page. These recommendations are based on the data collected in this study as a quick guide for interested cities or departments.

1. Create Goals - Prior to creating a page, the city or department should come together with any and all staff responsible for the Page and develop goals. These goals should focus on what the Page will look like, the "voice" of the page, internal and external protocols for terms of service, and security. Goals can be based on membership, such as achieve 1000 fans within three months of creating the page. Alternatively, goals can be based on feedback and interactions within the Page, such as achieve a $10 \%$ feedback rate for posted content. 
2. Terms of Use - Creating a terms of use protocol is important as it guides not only how administrators use Facebook, but also how users (fans) conduct themselves on the Page. Internal terms of use should focus on the "voice" of the page and ensure consistency. Although a formal "voice" is not discouraged, informal and relaxed communication is typical on Facebook as a it is a primary social networking site for friends. The external terms of use should be posted on the "information" section of the page and allow for fans to understand the rules and conduct of posting comments and/or media.

3. Content - Create interesting content that will engage fans. Content can be in the form of text or other media such as photographs or video. Providing interesting and informative content encourages fans to come back to the Page to see what is new. Content can also be in the form of links, such as news articles or website, and also external surveying websites to engage fans in public participation.

4. Updating - Updating the Page provides legitimacy of the Page and encourages fans to come back often to see new content. It is suggested updating at a maximum of three times per day, to avoid over saturating fans with information, and a minimum of once per week, to avoid a stagnating page and perhaps losing fans.

5. Promoting and Adverting - When a Page is created, it requires the administrator to promote and market it through online material as well as printed material. Online advertising can be free, as the "like" button and links to the Facebook Page can be posted on existing city websites, as well as on the signature line of employee emails. Promotion is necessary and although there are free alternatives, 
Facebook provides a paid service. Administrators can use the Facebook advertising tool to reach a specific audience based on their location, age, gender, and/or interests. Any type of marketing should be discussed at the beginning of creating the page as a part of the initial goal-setting meetings.

\section{Why use Facebook?}

There are various reasons to create a Page as outlined within this study, however statistics show that there are over 500 million active users on Facebook of which 50\% log onto Facebook in any given day. Additionally the time spent on Facebook is equal to over 700 billion minutes per month worldwide. Therefore not only are there a lot of users, but users are on the site consistently. Pages generate attention of the users with the average users connected to 80 community pages, groups, and events. Facebook also provides more than 70 translations which may be attractive to planners in multicultural cities. In addition, Facebook not only can be accessed from any computer, but mobile devices with the internet can connect to a mobile version of the site. Conversely many smart phones (e.g. the Apple iPhone, any Android based phone, Windows phone, and Blackberries) have dedicated applications that allow users to upload content remotely. Planners may

use this feature when in the field or on a project site to take photos of progress and upload them for fans to see.

Facebook is one of many choices for social media, however it is one of the largest social networking sites available. Because of its size and rate of new users per day, it is an ideal tool for planners to employ as a way to stay connected with residents. The Page allows planners to directly and indirectly contact people and not only share knowledge and media, but also receive input. Planners should be aware of other forms of social 
media/networking as it is an ever evolving technology that may not have reached its true potential as a networking tool. Again, Facebook has been the most dynamic form of social networking, starting off as a profile sharing website into a full networking site to include professions, businesses, and the United States Government. The Pages feature allows for organizations to take part in the highly saturated personal profile arena and essentially act as a "friend" of any user. In other words, the pages provide seamless integration within the existing network of personal friends. Building trust as a page is vital and ensuring that fans know the city has created and official page is essential. Creating and maintaining a Page can be achieved at no cost to the planning department other than the staff hours. Facebook also ensures intuitive controls and options that allow any user who is comfortable with basic computer functions the ability to create a page. Facebook.com provides guides to creating a Page on a basic level so that users feel confident and comfortable with venturing into making a Page. However, the guide for creating a page is very general and thus this manual provides information on how to create a Page specifically for cities.

\section{Creating a Facebook Page}

The process for creating a Page is relatively easy, however there are some issues that planners must be aware of before going forward. These are:

1) Permission - Depending on who initially decides to use Facebook as a tool for the planning department, there may be the need to gain permission from managers or the director of planning. Everyone in the planning department should be aware of the choice to move forward with a Facebook Page, but essentially decision makes within the department should meet with potential administrators and the Information 
Technology department to develop goals, create administrator login credentials, and ensure the department has access to Facebook.com on their computers.

2) Develop Goals - Various types of goals can be set by the administrator and the department as a whole. These goals should be discussed with all the necessary staff to ensure proper implementation and measurability. Although a common goals is to obtain a predefined amount of fans within a certain amount of time, there may be other definable goals that meet the criteria of the department. Other goals may be a particular response rate or "feedback" rate per post. Facebook provides this data in real time and allows the administrator to see what produces the most responses as well as which posts fans view the most. Goals of this nature are independent of the number of fans the page has and therefore may be useful for planners. A large fan base does not necessarily mean a "better" Facebook Page, instead an active fan base provides for interactions and a sense of public participation.

3) Creating Administrator Credentials - Facebook requires each page to be created by a user with a personal profile. The "Pages" feature augments the personal profile aspect of Facebook and as such requires a username and password. cities should be aware that a personal email address is not recommended. This is due to the fact that in case the password is ever lost, a recovery password will be sent to the email provided. Login credentials are in the form of an email address and password, not the username and password. Therefore, the Information Technology department should create a specific email address for the administrator that conforms to the same format as typical city employee email addresses. This may look like:

\section{[City name]Facebook@ cityname.com planningfacebook@cityname.com}




\section{fbadmin1@ cityname.com}

Please note that if there are multiple administrators (recommended) then there will need to be a dedicated email address for each administrator. It is not recommended that a city use a single administrator login for multiple users. Because the Page will represent the city as an official entity on the website, accountability for posting is essential. Staff must be aware of what they are posting and to follow guidelines for uploading content to ensure 1) consistency of content, and 2) appropriate content that represents the goals and mission of the city.

Choosing the administrators is important and essential to ensuring the security of the Page. Therefore at the time of creating administrator credentials, the city should decide who will have the responsibility and create or follow an internal terms of use protocol. It is recommended that the administrators have the same rights and responsibilities as those who have permission to give a press release or speak to the media. Many cities follow this recommendation and trust staff at an executive level with the responsibility of maintaining the page. This is a recommendation and not necessarily a requirement, as many cities have not had issues with staff posting inappropriate content. However, some cities have reported that spelling and grammar have been an issue from some staff members and as such their superiors were responsible for removing, editing, and reposting the content. Adding and removing administrators is a relatively simple task and can be done from any computer connected to the internet.

4) Department Access - Some city departments block Facebook.com from work computers. This may be overlooked when initially developing a page and it may take 
days or weeks for the Information Technology department to allow limited access to specific users. It is therefore recommended that the Information Technology department be involved from the beginning to ensure a smooth transition into using Facebook from work. One city reported this as an issue and that staff was responsible for updating and maintain the page during personal time. This method did not provide the results the city was looking for and soon after administrators could access the Page from work. Although this manual focuses on Facebook specifically, if the department decides to venture into other social media sites (such as Twitter or YouTube) then it is necessary to gain access to these from work. Therefore, within the social media goals, additional services and websites should be listed so that the Information Technology department can create network permissions for those responsible.

\section{Steps to Creating a Page}

The following steps and associated screenshots provide information on creating a page for those who may not have experience with Facebook. Please note that the process for creating a Page is different than the process for creating a personal profile page.

1) It is assumed that the city has signed up for Facebook with the login credentials developed by the Information Technology Department. Once an email address and password are established with the site, login (item 1 in figure 19) 


\section{facebook}

\section{1}

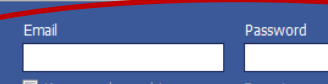

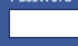

Heading out? Stay connected

Visit facebook.com on your mobile phone.

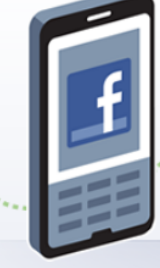

Sign Up

It's free and always will be.

Get Facebook Mobile

First Name:
Last Name:

Your Email:

Re-enter Email:

New Password:

I am: Select Sex: $\square$

Birthday: Month: $\square$ Day: $\square$ Year: $\square$ Why do I need to provide my birthday?

Sign Up

Create a Page for a celebrity, band or business.

Figure 19 Facebook Login Page

There is also the option to create a page directly from this home screen (item 2 in figure 19). This option will still require login information at a later step and to ensure the simplicity in this step-by-step section, login at the top to access your personal profile page.

2) Once logged in, you will be presented with your personal profile's "News Feed". This is a section dedicated to providing updates from friends and/or pages that the administrator is a fan of. Customizing a personal profile is not necessary to run the Facebook Page; some cities may opt out of this feature and keep any activity strictly on the Page and not the personal profile. Scroll down to the very bottom of this page until the links shown in Figure 19 appear.

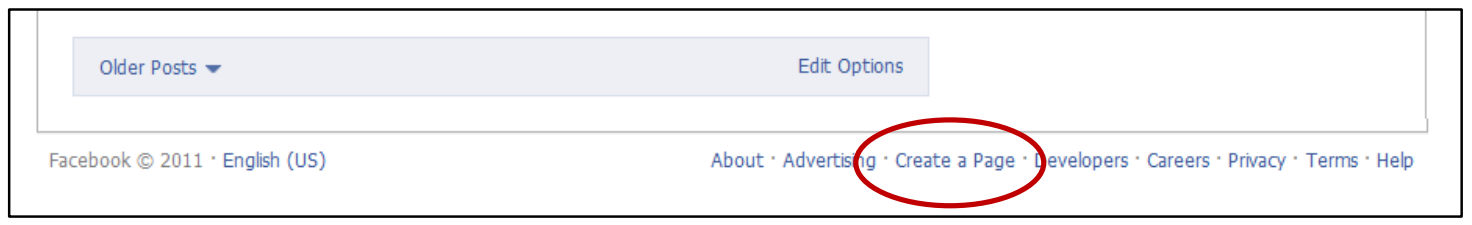

Figure 20 Create a Page Link 
The "Create a Page" link is at the bottom of all Facebook webpages and so is easily accessible. Select this link to move onto the next step.

3) The Create a Page link brings up a selection of six general types of pages as seen in Figure 21.

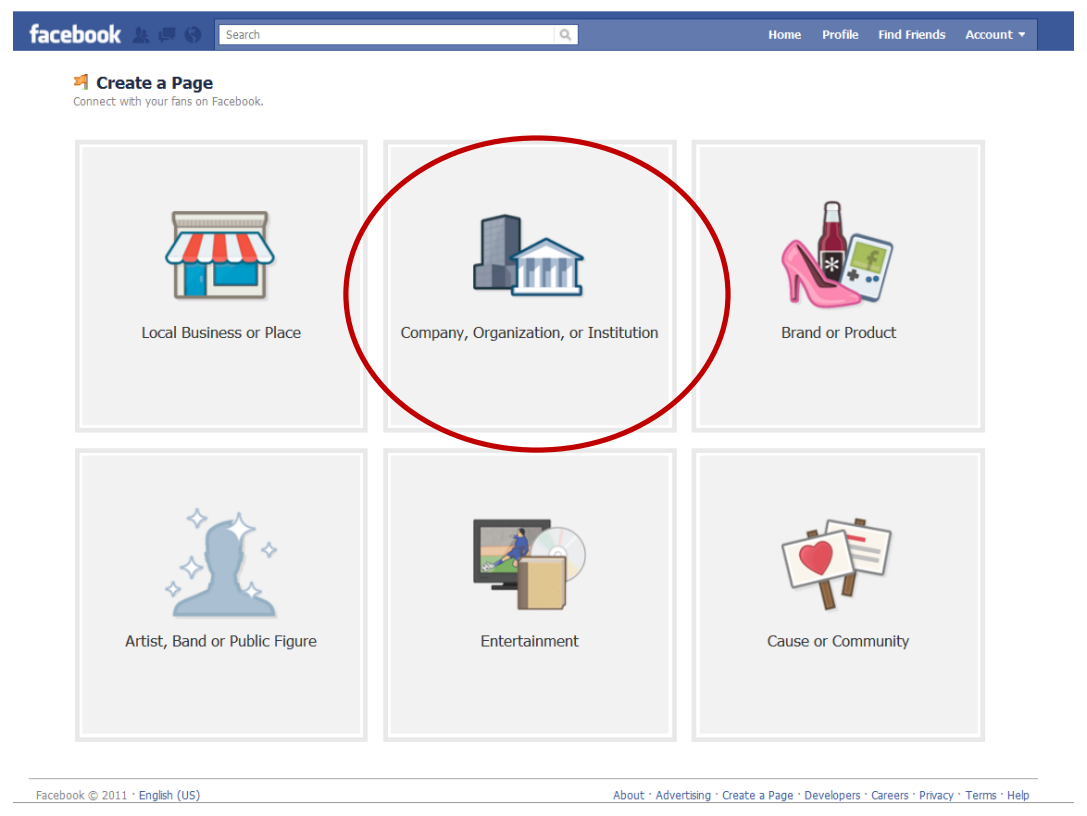

Figure 21 Create a Page Selection

Select the "company, Organization, or Institution icon to create a government organization page. Figure 22 shows the sub categories available once the icon is selected. 
Company, Organization, or

Institution

Join your supporters on Facebook.

Choose a category

Choose a category

Aerospace/Defense

Automobiles and Parts

Bank/Financial Institution

Biotechnology

Cause

Chemicals

Church/Religious Organization

Community Organization

Company

Computers/Technology

Consulting/Business Services

Education

Energy/Utility

Engineering/Construction

Farming/Agriculture

Food/Beverages

Government Organization

Health/Beauty

Health/Medical/Pharmaceuticals

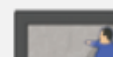

Figure 22 Organization Subcategories

Select the "Government Organization" subcategory and then decide on what the name of the Page will be and check the box to agree with the terms of service (not show in Figure 22). Once this is decided, select the "Get Started" button to establish the Page.

4) The Get Started page provides six steps to establish a very basic page (Figure 23).

The options to add a profile image, invite friends (as fans), import contacts, post a status update, promote the page, and set up your mobile phone are listed as the initial steps. Additionally, the user who created the page is now the administrator and has full rights to the page. Located on the top right of the page are account and profile tools (as of June 2011) where the administrator can select "Use Facebook as a Page" which should be noted for future reference. However at the current screen the administrator will want to setup the basic features, beginning with the photo. 


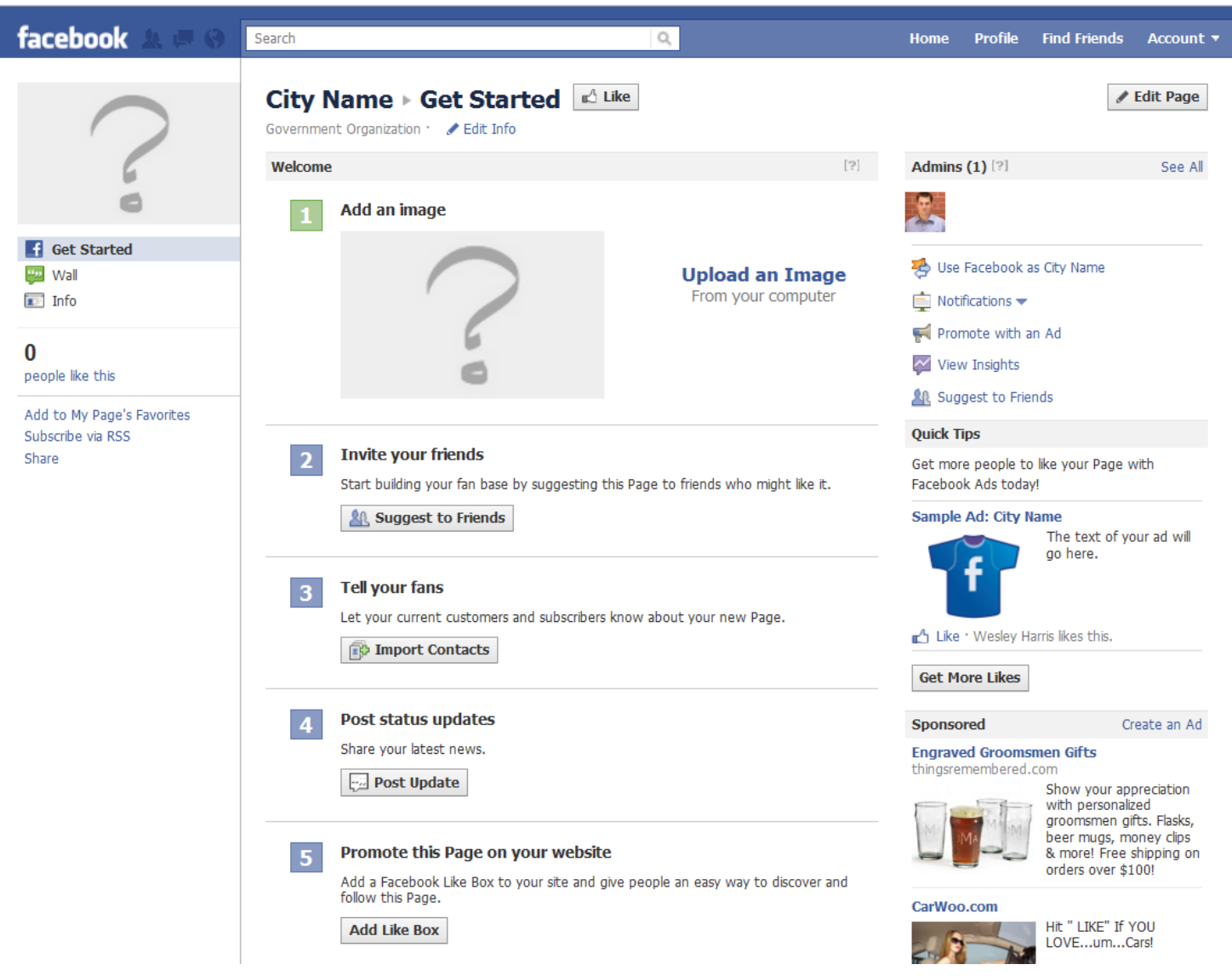

Figure 23 Get Started Page

5) Adding a photo is important to the Page and selecting an acceptable photo is necessary for setting the "tone" or "voice" of the page. Although there is no formal study that dictates the optimal photo to use, it is suggested that an iconic photo of the city is used as opposed to the city Seal. Essentially an iconic photo of a building or landmark that represents the city should be used to show potential fans a glimpse of the city as well as provide a visual aid for determining the nature of the fan Page. Remember the photo is reduced to a thumbnail size when using the search bar or when status updates are posted in the News Feed. The city Seal may not be as identifiable as a landmark or iconic photo when fans browse through hundreds of pages. Additionally the text will be unrecognizable at a thumbnail level and may 
cause potential fans to overlook the page when searching. Also, a city Seal represents a formal government, whereas the culture of Facebook is typically informal.

Facebook is a place for friends to find each other and network, and so creating a formal or serious "voice" may push some fans away. Figure 24 is an example of the City of Atascadero's Page, whereby the historic City Hall was selected as an identifiable profile picture.

\section{facebook \& $\Leftrightarrow 5$}

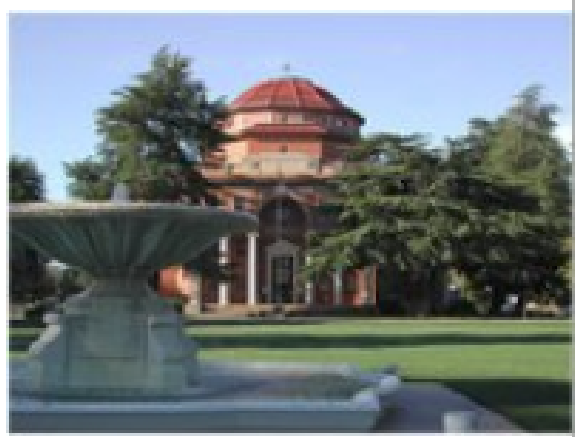

\section{Get Started}

Figure 24 Example of an Iconic Photograph for the Profile Picture

6) Once a photo is selected, the administrator will need to edit the page via the "Edit Info link" or "Edit Page" button (see Figure 25 for locations).

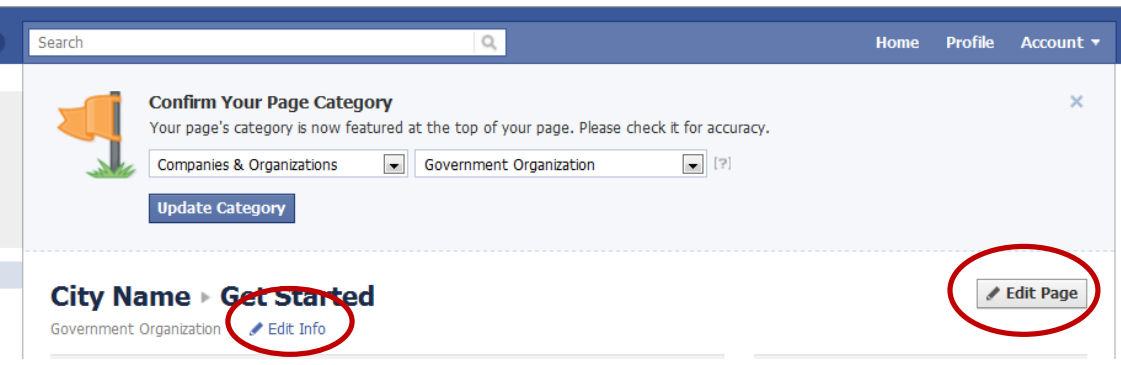

Figure 25 Edit Page and Edit Info Locations

The next page displays a navigation bar to the left with the various options for editing the Page (see Figure 26). The first section listed on the navigation bar is called "Your 
Settings", located here are two options to determine how content is distributed and how the administrator is notified. These two settings are up to the administrator to decide and do not affect content of the Page.

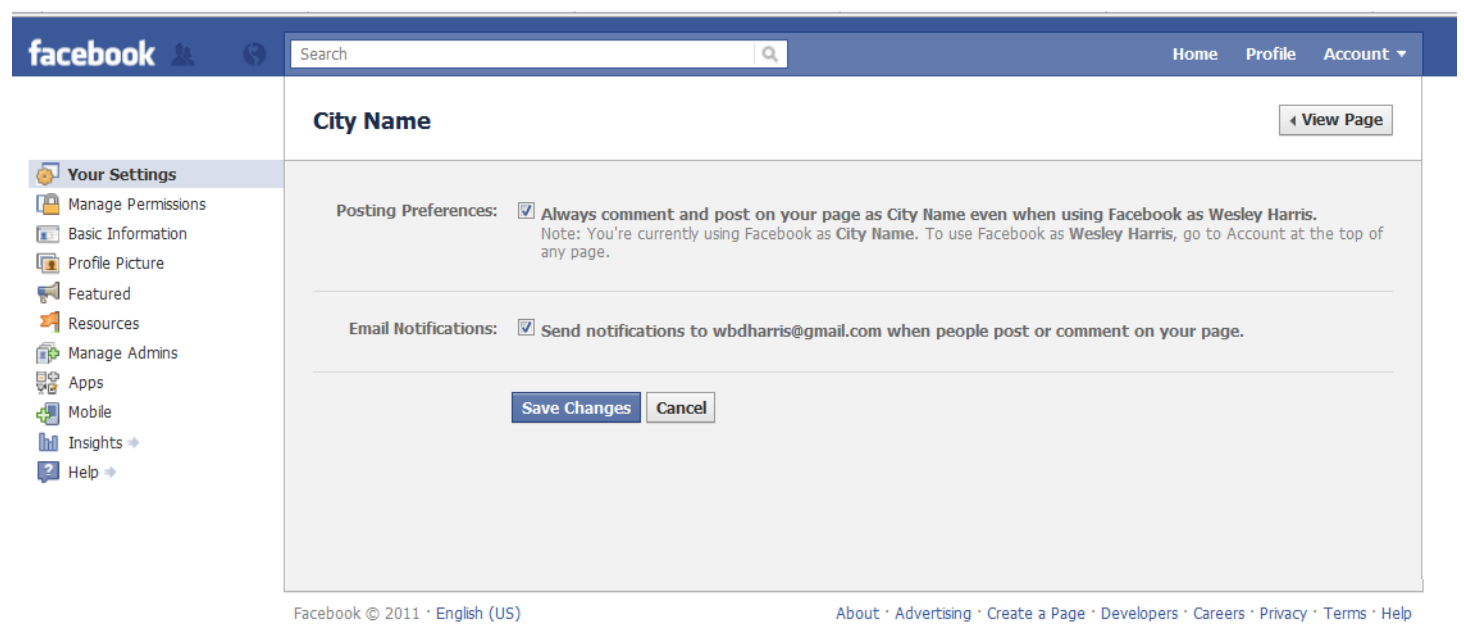

Figure 26 Facebook Page Settings/Navigation Bar

7) Manage Permissions - This section provides settings that will directly affect the Page and should be reviewed carefully. When setting up the page for the first time, select the box next to "Page Visibility" so that only administrators can see the page. Do not uncheck this box until the page is complete and ready for public launch as anyone can view the page if this box is not checked.

The next setting is the "Country Restriction", this can be selected to ensure only those in the United States can view the page. This setting is only restricted to country and not any other geographic level (i.e. by city). This option is at the discretion of the Administrator although a recommendation would be to set it to the United States in case to limit any and all spam origins to a single country.

"Age Restriction" allows the administrator to set limits on the age of those who view the page. Because the page will be an official form of city communication, 
keeping the minimum age restriction (13+) is recommended so that certain age groups are not limited from participating.

The "Wall Tab Shows" selection is only important if the administrator does not limit commenting. Comments made by fans can be unlimited, essentially allowing them to post on the city's wall arbitrarily. Or comments can be limited to only in response to city posted content. If unlimited commenting is allowed by the administrator, then the "Wall Tab Shows" selection provides for all content (fan and city) being displayed on the wall or only city generated content. If the administrator decides to limit commenting, then this feature is irrelevant and will default to showing only city posted content.

The "Default Landing Tab" translates to "which section of the page should be displayed when fans visit". Depending on the amount of sections (tabs) that the page has, options to "land" on those sections will be available. It is suggested that the wall is the default landing tab because this is where most of the content is posted and where updates occur. Most cities use this as a default landing tab unless there is a tab created specifically for a promotional item or event and therefore the city wants to showcase.

A very important setting is "Posting Ability", where this allows the administrator to limit commenting and media sharing. It is up to the discretion of the city to decide if they would like to include unlimited commenting and/or media sharing on the Page's wall. The check boxes are self-explanatory and allow for comments, photos, or videos to be posted. It is recommended that cities unfamiliar with creating and maintain a Page turn all abilities to post off. However, fans are still permitted to 
comment on any update that the city posts. This is because Facebook does not allow Pages to limit all commenting in order to keep the page a social media tool (two-way conversation). If at any point the city and administrator feels comfortable for allowing commenting and posting of media, these boxes can be checked.

The rest of the settings in this section are dedicated to limiting content by keywords. The "Moderation Blocklist" allows for the administrator to list words that may be considered spam or inappropriate for the page. Additionally the "Profanity Blocklist" uses an automated system to immediately block comments with profanity. These settings may be left blank for the initial creation of the page and should be edited if profanity and spam become a major issue with maintain the page.

The final setting in this section is "Delete Page", as the name implies, this is where the administrator can permanently delete the page.

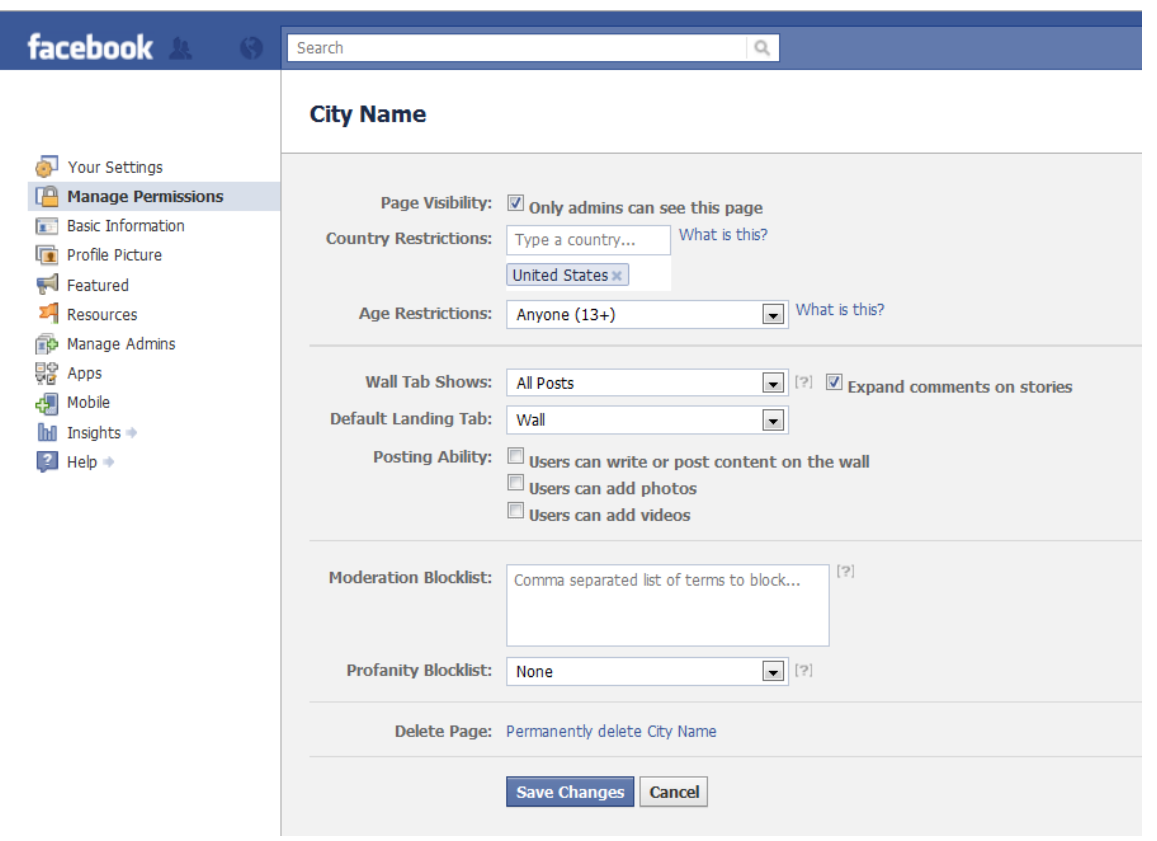

Figure 27 Manage Permissions Settings 
8) Basic Information - This section allows the administrator to enter information about the city. Information entered here is presented in a section called "Info" in the navigation bar of the Page (on the left side of the webpage under the profiled picture).

The "Category" of the Page that was initially selected can always be edited and changed to represent the type of Page. This should not be changed unless "Companies \& Organizations" and "Government Organizations" are not already selected.

The "Username" is another very important aspect of the Page. Here is where the administrator will ultimately select the username of the Page that is directly after the Facebook.com URL address. A username provides an easy way for potential fans to access the page. An example of a Facebook page username is as follows:

\section{www.facebook.com/cityusername}

Without a username, the URL for the page will be long and incorporate a mix of numbers and letters that may be difficult for people to remember. There are two caveats with regard to usernames. The first is the page requires a minimum of 25 fans before a username can be selected. The second is once a username is selected it cannot be changed, transferred, or removed. The username is permanent and even if the Page is deleted, the username will not be available for security reasons. Therefore it is imperative that a correct username is selected and that it is created under the Page username and not the personal profile name. This is a very important step as any mistake in the username will be permanent and thus force the administrator to create a new Page in which another 25 fans will be required before the ability to select a username is available again. 
The "Name" section refers to the title of the Page and is useful when searching for the page in the search bar. The name should be representational of the planning department in the same way the profile picture represents the city. The name can be changed at any time and is not directly related to the "username" and so could be completely different than the username.

The options available after the "Name" section refer to specific information about the city/planning department. It is recommended that the administrator enter as much information about the department as possible in this section. Information such as address, phone number, or website could potentially answer questions from fans. Information entered within the "Description" and "General Information" sections are at the discretion of the administrator, however it is strongly advised that the "General Information" section include a terms of service message for fans to review (see Terms of Service subsection in this manual). Providing a lot of background information in this section promotes transparency as well as giving the page a "voice".

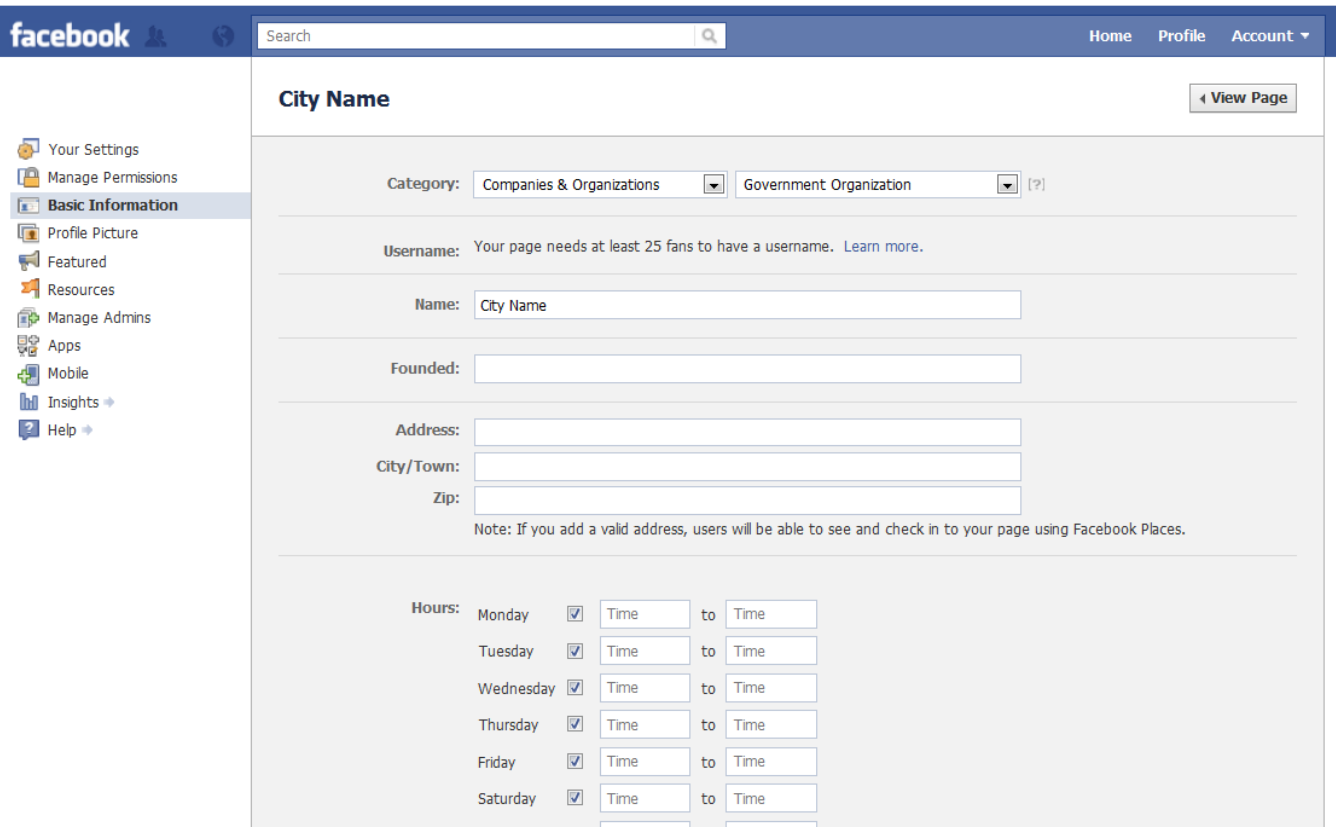

Figure 28 Basic Information Settings 
9) Manage Admins - This section is where administrators can be added and/or edited. It is important to note that additional administrators must be friends of the personal profile, or existing fans of the Page. Once all the potential administrators are fans of the page, it is simple to enter their names/email address and select them as administrators. Please note that the "only administrators can see the page" in Figure 27 , must be deselected so that potential administrators can see the page and thus add it. Therefore, the primary administrator must ensure the page is complete (sans additional administrators) before adding any new administrators.

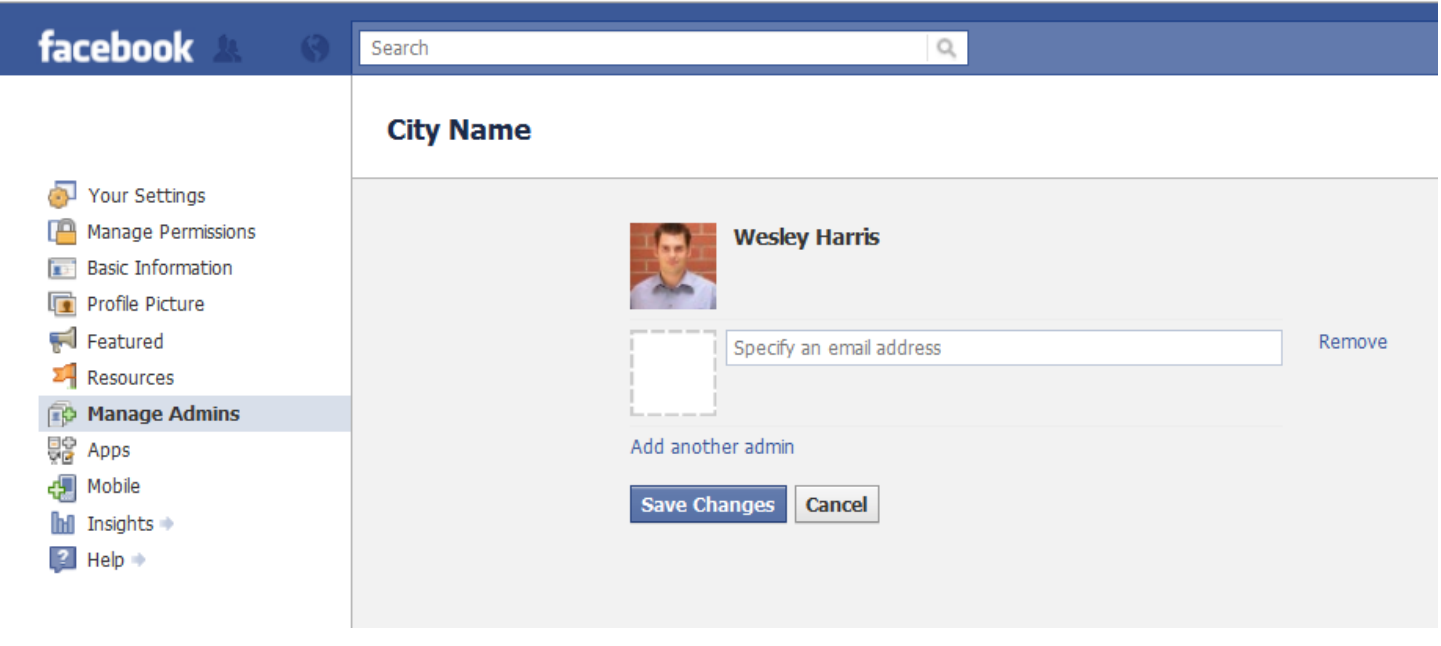

Figure 29 Administrator Privileges

10) Apps - The apps (short for applications) section displays potential applications for the administrator to add to the Page. These applications range from media integration (such as the Photo app), to an events section. Ultimately it is at the discretion of the administrator to determine which applications to include on the Page. More applications essentially mean more content to create and/or monitor. Therefore for new Pages, the basic applications are recommended:

- Photos

- Events 
- Discussion Boards

These four applications are available within the apps section of the settings and additional applications can be found via the "Browse more applications" link at the bottom of the list (Figure 30).

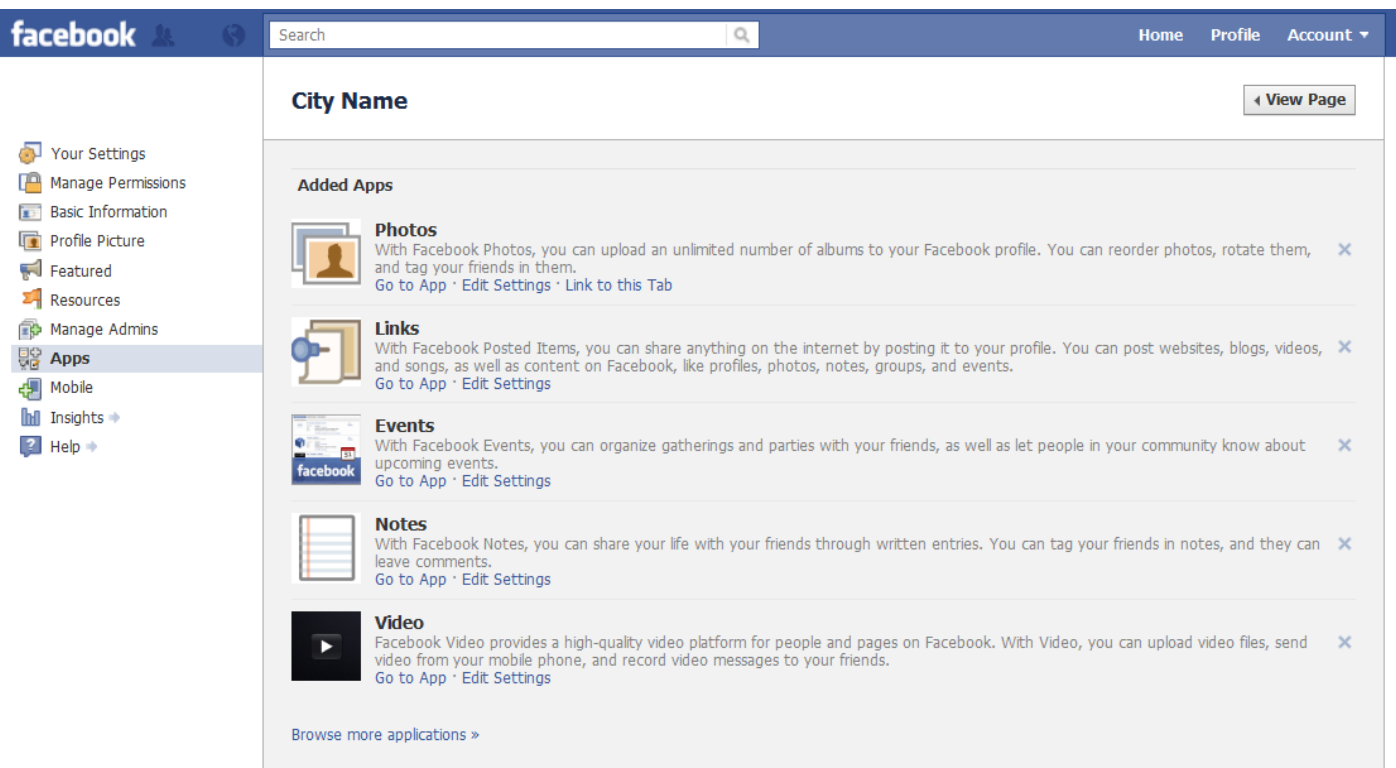

Figure 30 Application Selection

The photo application is self-explanatory, by selecting this application the administrator creates a section dedicated to uploading and viewing photos. Fans can browse photos via albums and photos can be tagged. A tagged photo means that the administrator clicked on a photo and attached a description or a fan, or another Page. Tagged photos are great for cross promotion of multiple Pages as tags are links to other pages or fans. For example if there was a photo of a city park, the administrator might tag the Parks \& Recreation Page within the photo and thus create a link from the photo to their fan Page. Additionally, photos that the planning department is tagged in appear in the album under "tagged photos". However, the administrator can limit who tags photos of the planning department within the "Photos" application 
settings. Administrators may choose not to allow fans to tag photos of the planning department and is a recommended setting for new Pages. There is potential for spam and advertising if fans are allowed to tag photos of the planning department/city in photos. Caution must be exercised when deciding to use this feature and as such, well established pages may consider the notion.
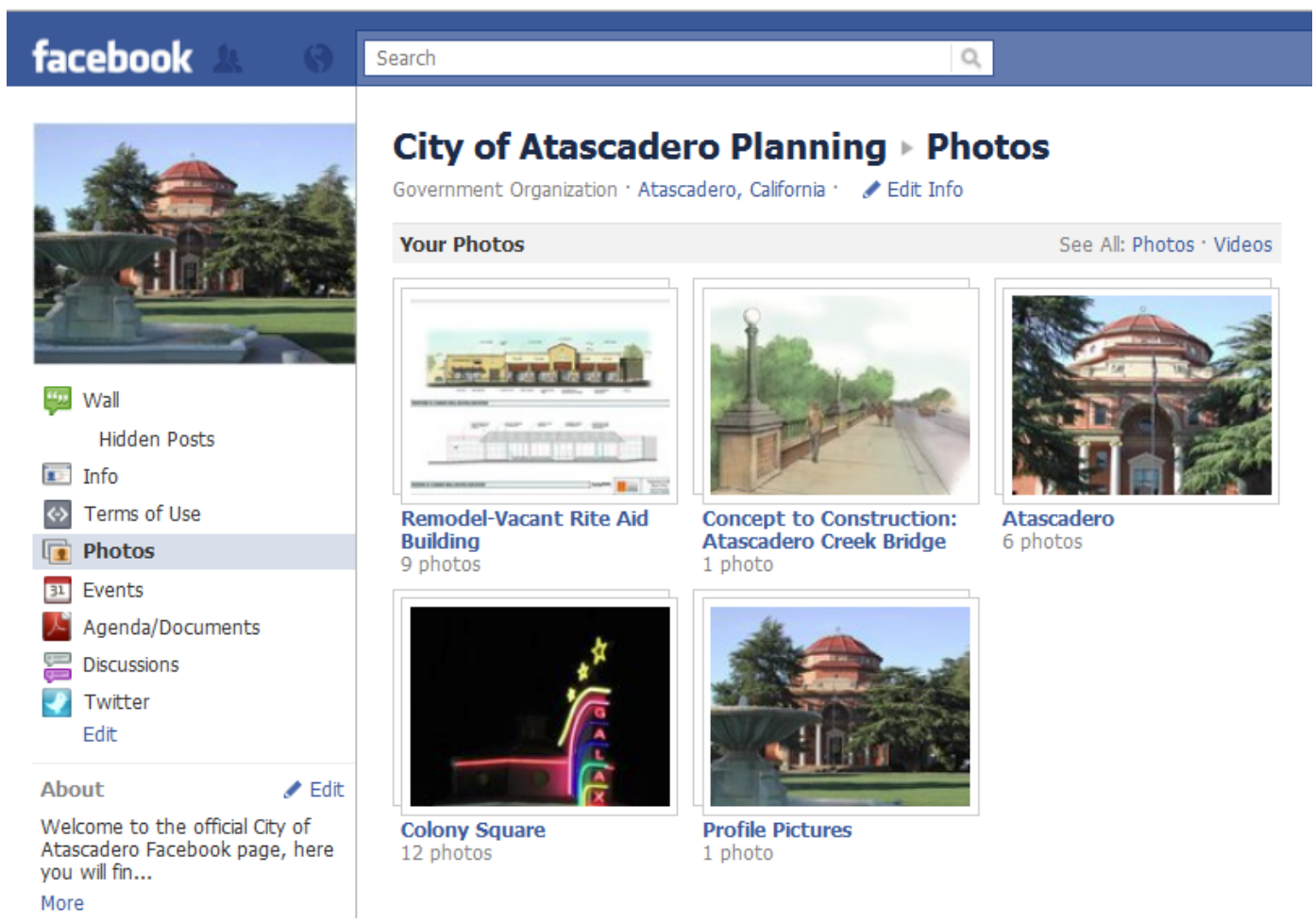

\section{City of Atascadero Planning - Photos}

Government Organization · Atascadero, California · Edit Info

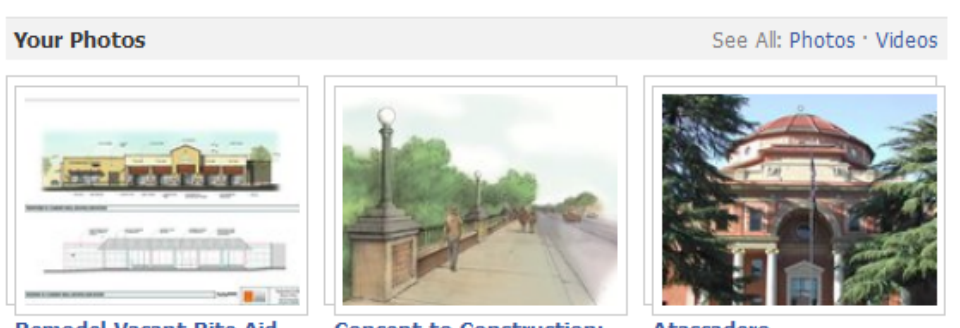

Remodel-Vacant Rite Aid Concept to Construction: Building Atascadero Creek Bridge

Atascadero 9 photos 1 photo

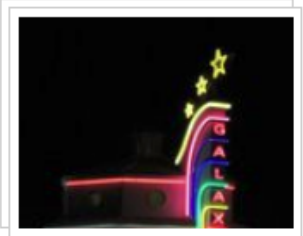

Colony Square 12 photos

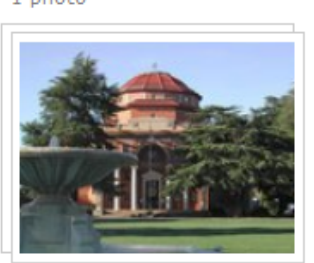
6 photos

Profile Pictures 1 photo

Figure 31 Sample Photo Album, City of Atascadero

The events application allows for the administrator to create specific event details and invite fans (Figure 32). This section provides an opportunity for actual public participation through notice of a public meeting/workshop within the Page. The administrator has the options of a time, place, and description of an event. Additionally events are open to fans to respond to with both the ability to declare if they are attending or not as well as the ability to comment on the event itself (Figure 
33). Events are posted on the wall as well as the dedicated event section located within the navigation bar on the left of the Page.

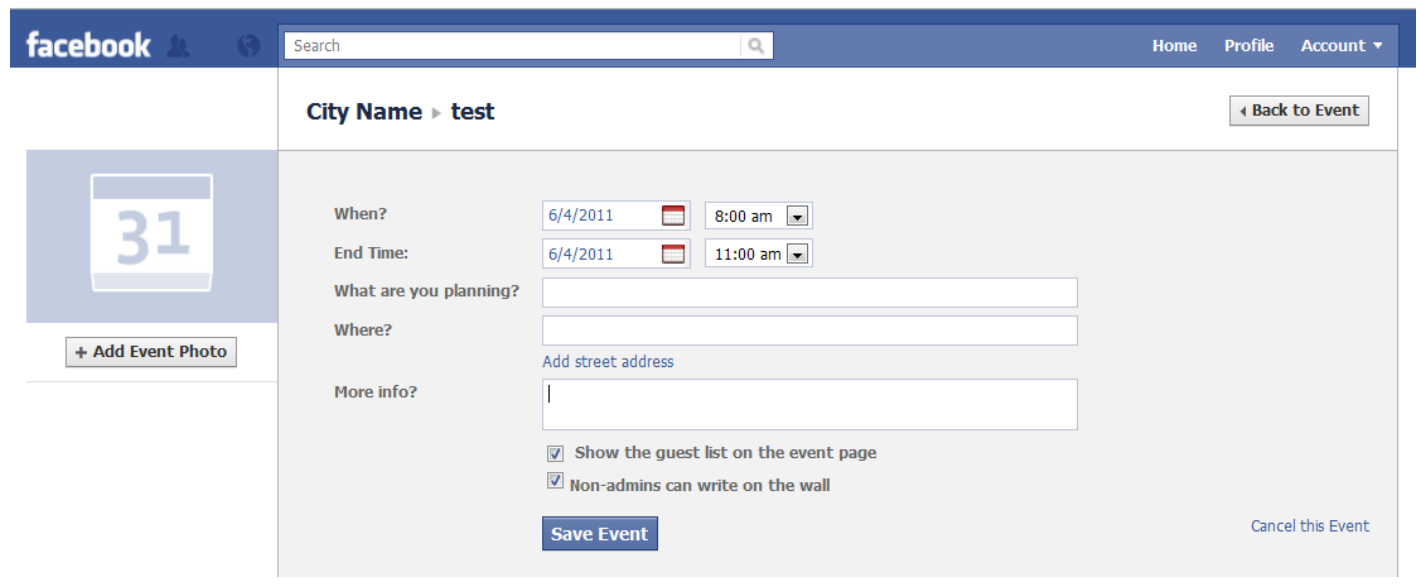

Figure 32 Creating an Event

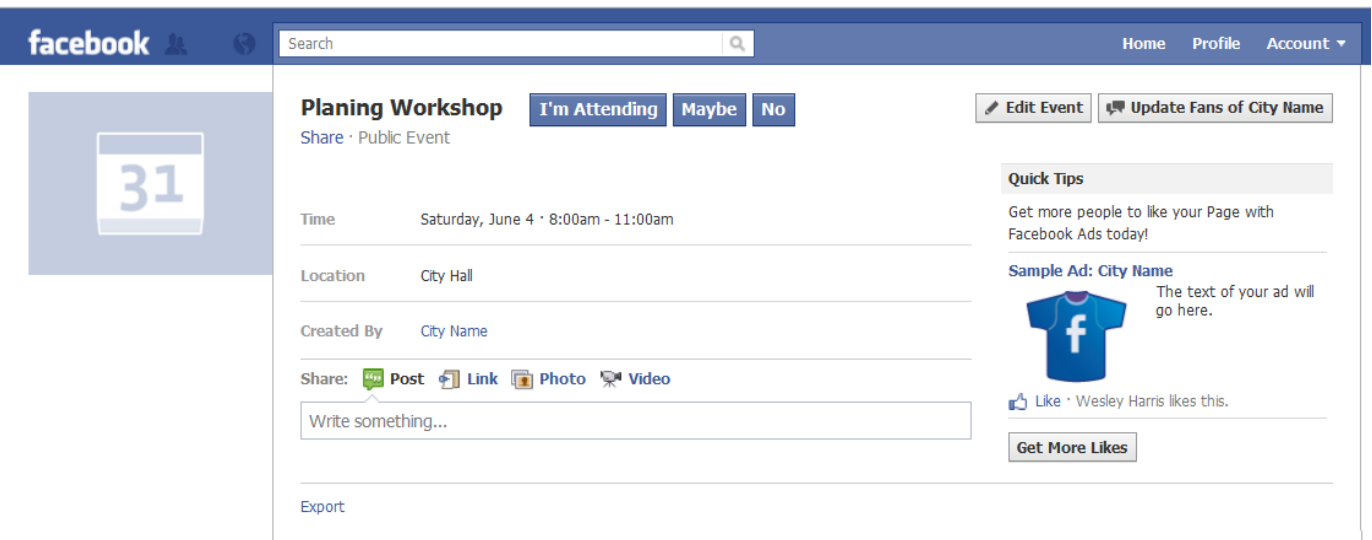

Figure 33 Event Details as Viewed by Fans

Discussion Boards provide a dedicated section of the Page to discussions about specific topics. This is different from posting content on the wall and having fans respond, as this application provides an internet forum-like interface. Discussion are organized into topics and fans can visit and comment on various discussion items. It is suggested that if the wall is being used as main area for discussion or debate on a single issues, then create a discussion topic. The wall is useful for updates and quick responses from fans to their preferences and "likes", but debatable discussion items 
should be given a dedicated area for public comment. However, this requires additional moderation of content. It may not be feasible for the administrator to monitor both the wall and the discussion section. This is at the discretion of the administrator, but the recommendation is to create topics for debatable items.

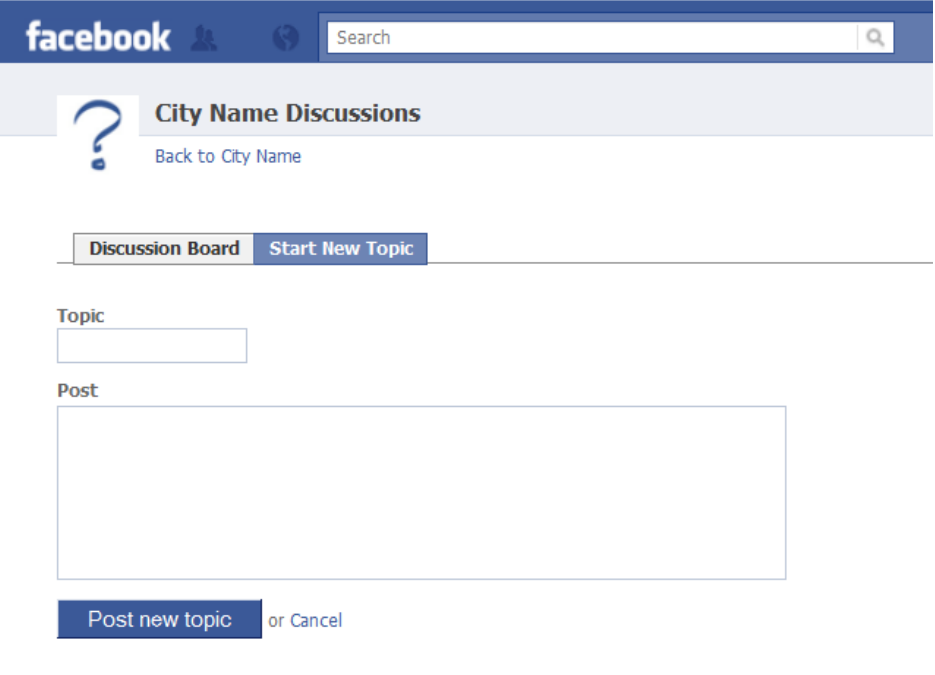

Facebook @ $2011 \cdot$ English (US)

Figure 34 Discussion Section

11) Terms of Use- It is suggested that the administrator develop a "Terms of Use" protocol for fans to follow. Because the page represents the city and planning department, moderation of content is important. Like many website, Facebook has fallen victim to spam and copious amounts of advertising. Many cities have encountered this phenomenon and as such create Terms of Use protocol to justify the removal of specific comments. In addition to advertising and spam, the administrator should reserve the right to remove profanity and explicit comments. There are no typical terms of use templates, however the City of Atascadero provides a good example. This is an important step in creating a planning Page as it guides the use of the page and sets restrictions on explicit, off topic, and advertising based comments. 
It is not recommended that the planning department remove negative comments.

These are distinct from explicit or inappropriate comments as they provide feedback from the public on concerning issues. However, there is a fine line between negative feedback and slander. Therefore the administrator should meet with the City Attorney to determine what restrictions can be put on commenting. Figure 35 provides an example of the City of Atascadero's Terms of Use.

City of Atascadero Planning $>$ Terms of Use

\author{
Government Organization · Atascadero, California
}

This is an official Facebook page of the City of Atascadero. For more information about the City of Atascadero please visit Atascadero. This site is intended to serve as a mechanism for communication between the public and the Community Development Department to further our mission. Any comment submitted to this page and its list of fans may be considered a public record which is subject to disclosure pursuant to the California Public Records Act. Public information requests must be directed to the City Clerk's office.

Any comments posted to this page will be monitored daily and inappropriate content will be removed as soon as possible. Under the City of Atascadero Social Media Use Policy, the City reserves the right to remove inappropriate content, including, but not limited to, those items that have obscene language or sexual content, threaten or defame any person or organization, violate the legal ownership interest of another party, promote illegal activity and promote commercial services or products. The City disclaims any and all responsibility and liability for any materials that the City deems inappropriate for posting, which cannot be removed in an expeditious and otherwise timely manner. The City reserves the right to remove any fans that continuously violate this policy. Please enjoy this page and if you have any questions regarding the terms of use please email us at planningfb@atascadero.org.

Figure 35 Example of Terms of Use Language

These 11 steps provide the administrator the basic tools and functions of a Facebook page. Additional research is suggested to add content and applications (such a Twitter portal, PDF/Document viewer, or video player) to expand the function of the Facebook Page. However, additional content and applications requires additional moderation, creation, and maintenance of the Page. It is essential that the administrator(s) are available to update and monitor the Page on a regular basis, otherwise there is a high 
chance that the page stagnation will lead to a decrease in fans and/or an increase in inappropriate content.

\section{Content - Type}

With the creation of a page in place, the primary administrator is required to post content. This content is in the form of a status update, photograph, video, or external webpage link. Content is the very reason the page gains viewership/fans and as such must be compelling and hold interests. Administrators and planning staff must ask themselves “why would our residents want to become a fan on Facebook?" This question may be associated with the initial goals of the page. For example, if an initial goal of the Page is to increase the fan base of the Page, then perhaps residents might want to become a fan because of detailed information about new projects in the city. The Page could be a hub for information about all the new projects coming to the city, where residents may want to know if a new Starbucks is moving into their neighborhood or if a 100 acre Specific Plan is being developed across town. Creating interesting content will keep fans interested in the page and it is assumed that they will respond with input (either positive or negative). This input is part of the public participation process and if the goal of the Page is to increase participation, then it is necessary to provide content to create conversations.

Research shows that cities will post a variety of content dependent on their focus and goals of the Page. Some cities release secondary content - this is based on department created press releases, which provides another outlet for the press release (other than the newspaper and/or website) as well as simplifies the content creation process. Press releases are formatted for public consumption and as such, the Page can 
convey this information without additional editing. Other content may be in the form of local newspaper links on planning related items such as a new project or policy. Updating via links allows for planners to save on time as the articles are already written and edited. Primary content such as photos of a project throughout construction requires more time to edit and produce, but provided fans with additional media to browse. This could be the same for including videos and is recommended for cities that already have a YouTube page.

An example of relevant and interesting planning related content is the City of Atascadero's photo updates with an album titled "Concept to Construction". The notion of this is to highlight current projects that have been approved for construction. This requires the administrator to scan the initial elevations/renderings and then photograph the construction progress. Residents can then see the process and the details associated with approving a project and hopefully comment or give feedback. Although the feedback is important, the process of giving feedback is superior as the page is essentially acting as a public participation facilitator. However providing consistent and relevant feedback is a challenge for some cities.

Finally, any content that is posted and has a title or short explanation will acquire a "voice". This voice is essentially the tone of the page; some cities choose to have a friendly informal tone for updating content so that fans feel a human connection behind the updates. Other cities have selected a more formal voice that ensures fans that it is an official government run page. A relaxed/casual voice is recommended because the nature of Facebook is typically informal and a place for friends to connect with each other. 
However a balance is needed to ensure fans that the page is an official representation of the city and as such slang and other extremes of informal language should be avoided. In review the following are essential to posting content:

- Update the Page with interesting content that will capture the attention of fans.

- Content can be in the form of a status update, photograph, video, or a link to an article or website.

- Experiment with the type of content, use Facebook's Insight tool as a guide for what content is viewed often and what has the most feedback.

- Develop content trends, such as a photo album that showcases projects from conception to completion. These trends can lead to a loyal fan base that will check back often to see any new progress.

- Create original content so that fans want to visit the page for inside information on planning projects, or create a questions and answering section of the Page for residents to participate in any way possible.

- Give the page a clear and consistent voice as fans will want to connect with the planning Page as if it was a friend. However do not become too informal and not accurately represent the city as a government entity. It is essential to provide a consistent voice where multiple administrators are updating the page. A guide for posting should be developed in the case of multiple administrators updating to ensure consistency.

- As with any publication, know your audience. It is important to know the demographics of your fans and to create content accordingly. Some updates may be more relevant that others depending on the fan base. 


\section{Content - Frequency}

Regularly posting new content is important to the vitality of the Page. Some cities struggle with this as information about the city or planning department is not always in a steady, constant, flow. Alternatively, other cities struggle with the time constrain on posting regularly where staff may be allocating additional time and resources to updating the page. Although there are no formal scientific studies on how often to update the Page, but some cities post as often and three times per day and others post at least once a week. Remember posting often allows fans to see the dedication to keeping them informed with new and fresh content. However, the other extreme is over-updating with slightly less interesting content. A balance is needed to post captivating content on a regular basis. Updating for the sake of updating will be obvious and fans may actually lose interest in the Page. Information in the planning profession ebbs and flows and as such should be collected and released on a consistent schedule. Some cities use an editorial calendar as done with other media or websites that require sharing information consistently. This is a recommended practice as a schedule will provide a plan to save relevant information as it comes across the desk of the administrator. Although planners should be aware of time sensitive updates to avoid the risk of keeping information about an event or project that might not be relevant if not released on the same day.

Updating the page requires time commitment form the administrator. Depending on the type of content, it may require more time than anticipated and cause a strain on the administrator. The act of posting an update is as simple as typing a short status and pressing the enter key. However gathering, organizing, and editing content such as photos, videos, or documents may require additional time and resources. When 
developing an internal protocol for posting content, planners should set goals for the amount of time spent on updating the page per day. There is a distinction between updating and operating a Facebook page versus a website. The main distinction is that Facebook allows for feedback from a dedicated fan base. Therefore time and resources of the administrator may be split between posting new content and responding to fans. Depending on the size of the fan base and the flow of updates, some cities may consider dedicating an administrator for posting and updating content and a secondary administrator dedicated to responding to comments.

In review the following is essential for frequency of updating:

- Find a balance between updating too often (saturating the wall) or not updating often enough (a stagnant wall). A typical update rate would be at least once a day to once every three days.

- Create an editorial calendar to plan updates and ensure consistency. Posting ten updates in one day then not posting for another week is not recommended. A steady, constant, flow of information will provide fans with an incentive to view the Page on a regular basis.

- Create an internal protocol for administrators to follow. This should include time and resources devoted to updating and set limits and expectations for posting content.

\section{Internal Terms of Use Protocol}

Some cities and departments have developed internal terms of use protocols that guide administrators with the process of operating the Page. However, some cities have not developed such a protocol and instead use discretion with posting content. An adapted 
terms of service protocol specifically for Facebook may not be necessary is there is an adequate protocol for updating the planning page of the city website. Nevertheless, it is recommended that the administrator follow a protocol that dictates the following items:

- The "voice" of the page

- Updating frequency

- Content type

- Responding to fan comments

- Moderating the Page

The voice of the page is typically the language used. It may vary from city to city or even from department to department. It is recommended that the voice be friendly and informal as Facebook is a site for friends. The protocol should indicate what language is appropriate, such as "Come check out our new photos of the downtown theater project" versus "Photos are available for your review and comment for the AMC movie theater".

The protocol should include a guide on the updating frequency that may follow and editorial calendar style. Devoting resources and time to updating the Facebook page is no different than assigning tasks to planners to fulfill during their workday. Although Facebook is a free service, staff-hours are not. Therefore efficient use of time is necessary to ensure the administrator is can accomplish other tasks within their job description (assuming the role of Facebook administrator is not a full time position).

Determining the type of content is also necessary to include in the protocol. Administrators must choose interesting content to capture the attention of fans, as well as ensure it represents the city and/or planning department. The protocol should guide administrators on posting content and establish boundaries of what can and cannot be 
posted on the site. This will generally follow typical protocol for press releases or any other type of media release by the department, to the public.

The protocol should also set out strict guidelines to responding to fans. All responses are considered public record, and the planning department is responsible for any information shared with the public. Guidelines for responding or not responding to certain comments are necessary to safeguard against miscommunication and other issues related to responding to the public as an official agency.

Moderating the page is necessary to remove explicit and inappropriate content from fans. The use of foul language should not be allowed and will be included in the Public Terms of Use guide featured within the "Info" section of the Page. If fans do not follow this guide then their comments may be removed. However, this does not include negative comments toward the department. Fair and balanced public input should be encouraged and it is expected that some residents will become a fan of the Page to be heard on specific issues. This is essentially informal public participation and should be considered useful information. Although, the protocol should guide administrators as to the definitions of inappropriate commenting and perhaps be developed in part with the city Attorney to ensure an open and transparent government run Page.

In review the following is essential for Internal Terms of Use Protocol:

- It is recommended that an internal "terms of use" protocol is developed to guide planners/administrators of the Page.

- Create an informal voice for the Page that represents the department while maintain a sense of professionalism. The voice or tone of the page is directly 
related to the choice of language and the protocol should determine the use of some words versus others.

- The protocol should dictate the time and resources needed to update the page. Division of labor may alleviate one administrator's role and allow for other planning related tasks to be completed during the workday.

- Provide a list of the type of content to be posted on the page. Following a press release protocol may be advantageous as it ensures that any information posted on the Page is for public consumption.

- The protocol should set criteria for responding to fan comments. The administrator responding is responsible for representing the department and the city, therefore choice of words and tone should be appropriately conveyed. On the other hand, the city may not respond openly to fan comments and instead respond in a personal message (recommended) or not respond at all (not recommended).

- Moderation criteria should be a part of the protocol to ensure a clean and professional page, free of advertisements and spam. Arbitrary censorship is not recommended and the definitions of inappropriate content must be created to set guidelines for removing comments. It is recommended that the City Attorney be consulted to determine what is legal with regard to removing content.

\section{Promotion and Advertising}

Many cities and departments operate the page with little or no budget. This requires marketing the page on the main city website and/or printed materials with the Page username URL. Although Facebook provides an advertising service that will reach specific users and promote the Page to them. Advertising does not ensure or guarantee 
new fans of the page, but in some cases it has led to a steady increase of fans until reaching a plateau.

Advertising is relatively simple on Facebook and the settings for this can be accessed through the main page settings (see Figure 36). Select the "Resources" section on the left of the screen and then select "Advertise on Facebook".

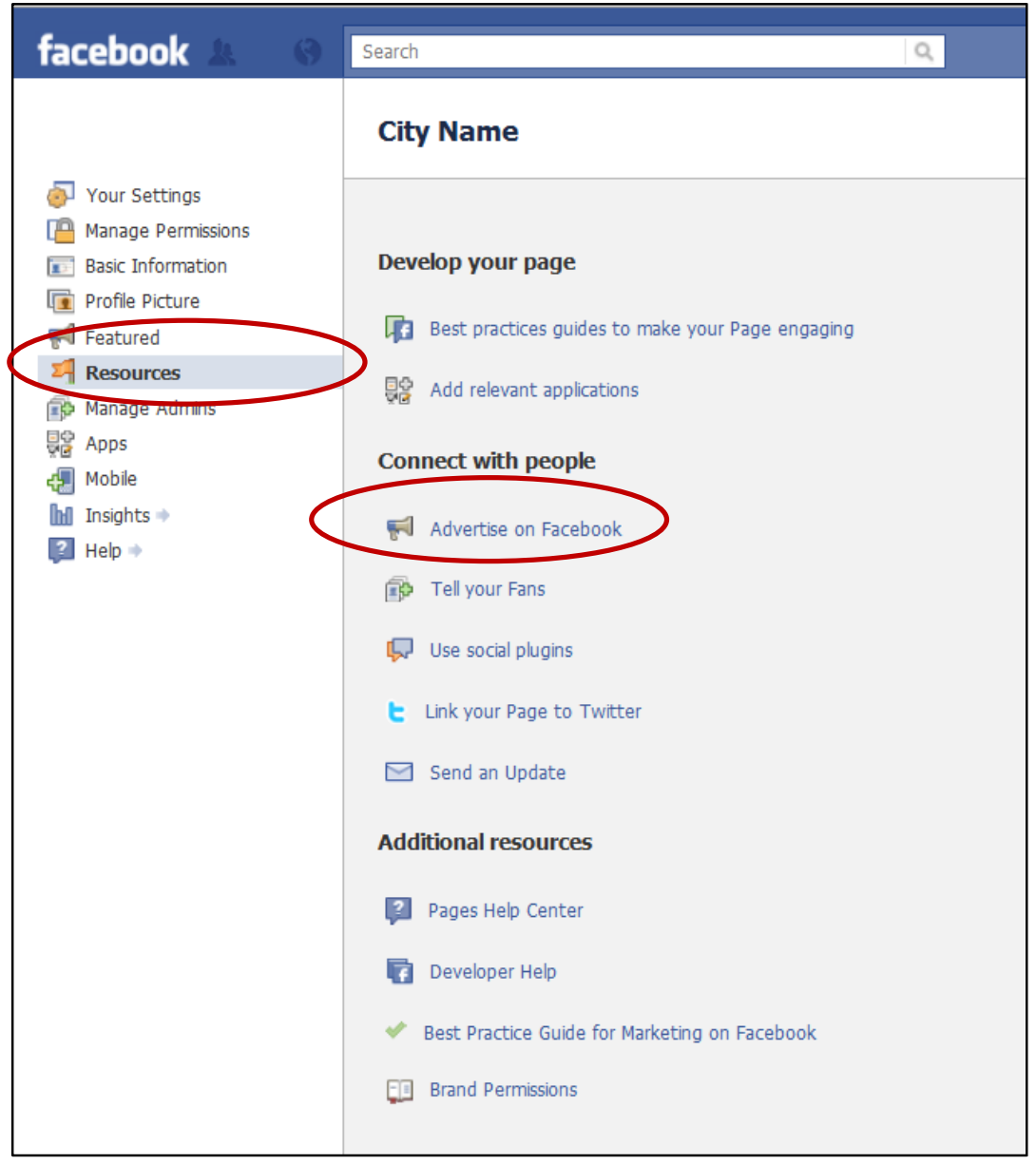

Figure 36 Advertising Settings

After selecting the Advertise on Facebook link, the administrator will be asked to switch to using the personal profile page. Follow these instructions to access the three steps to designing and marketing advertisements to potential fans. Designing the ad (Figure 37) should be at the discretion of the administrator and its best if all fields of step 1 are filled out completely. Facebook allows a preview of the ad to be featured either in the News 
Feed section or to the right of typical Facebook profile pages (the dedicated advertisement section).

\section{facebook \&\&}

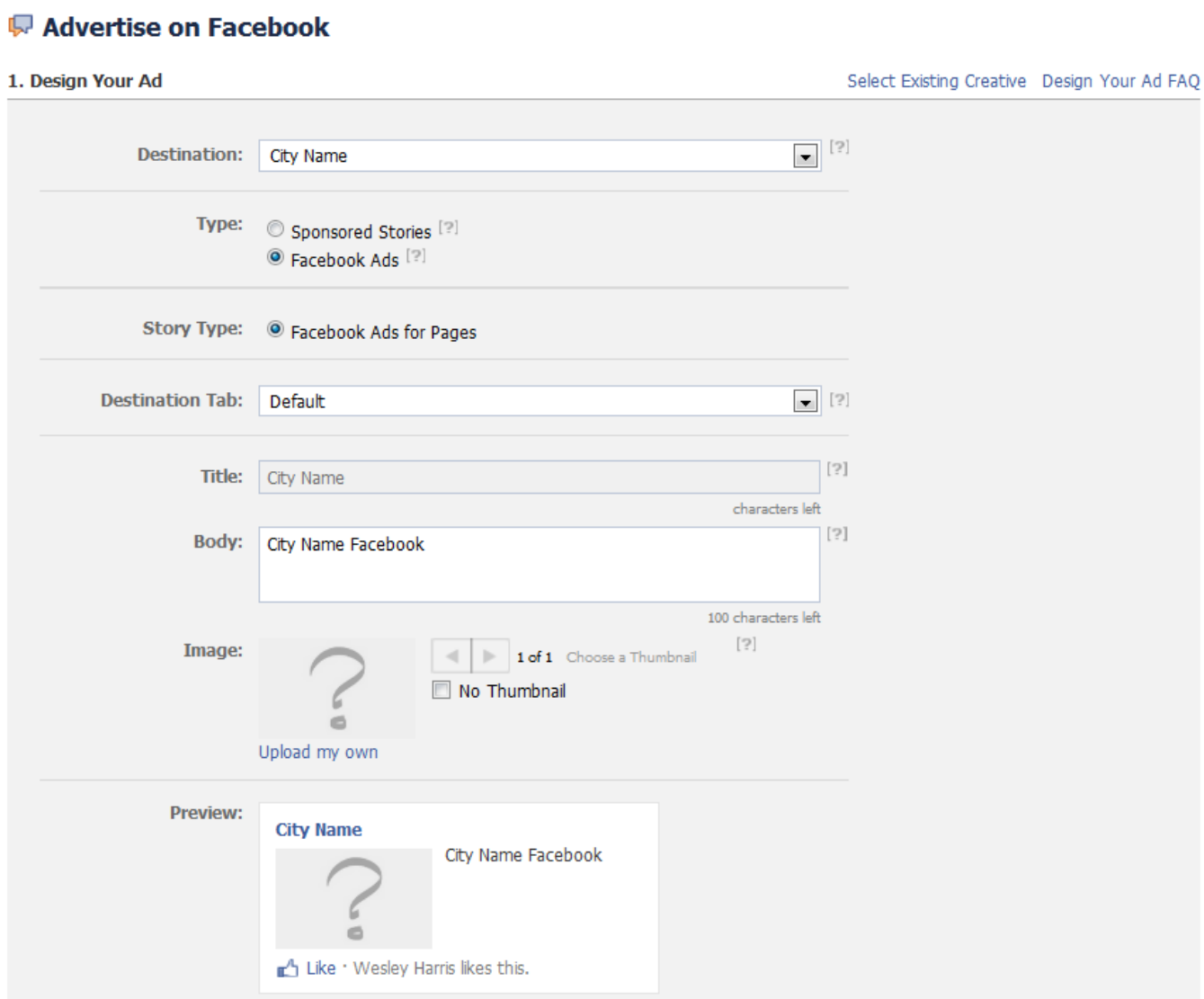

Figure 37 Step 1 of Advertising on Facebook - Designing the Ad

The second step for advertising is the "Targeting" section that allows the administrator to reach a specific audience (Figure 38). Users can be targeted via their location, age, sex, interests, and connection type. Ideally the planning department will want to select their city as the level for advertising. Selecting the "Include cites within X miles" is at the discretion of the administrator. Essentially those who want to be fans of the page and participate should be residents of the city. The age selection may be useful if current demographics of the page are lacking a certain cohort. This is at the discretion of 
the administrator and may not be a major concern and therefore the default settings should be used. The administrator also has the choice to target users who have specific interest. Because Facebook is a profile based service, users provide a lot of information about their interests and as such, advertising can directly reach specific people. This should be left blank as a default setting as advertising for the department Page should target residents of the city. Lastly, connections on Facebook provide settings for filtering out certain users. If the nature of the advertisement is to alert people of the creation of the Page, then "Only people who are not fans" should be selected. Advertising funds will be wasted if existing fans of the page are targeted. It should be noted that to the right of the targeting step will be a real time calculation of the potential audience. Administrators should review this and ensure they are targeting the correct location and/or groups of people.

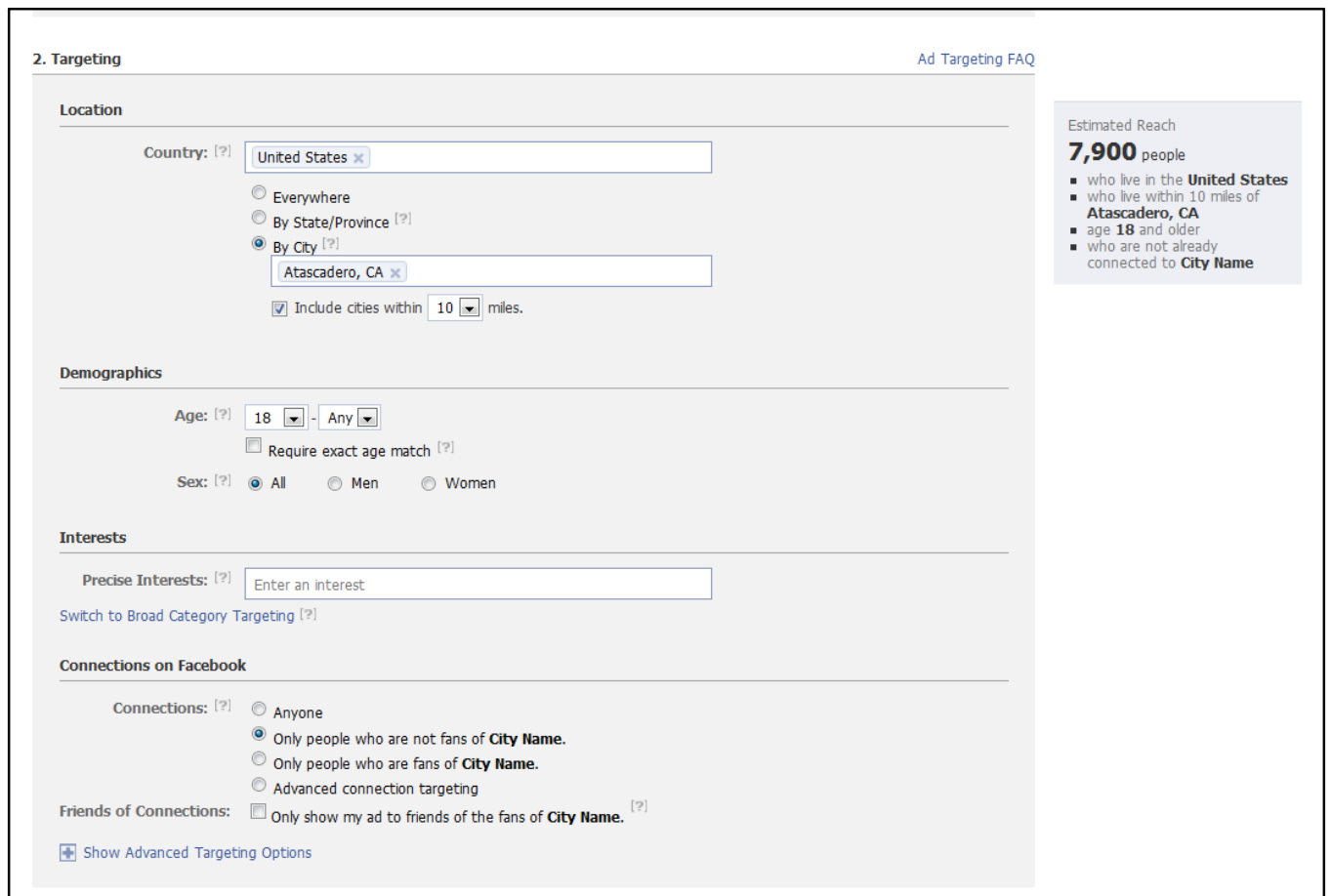

Figure 38 Step 2 of Advertising on Facebook -Targeting and real time Estimate Reach Statistics 
The final section "Campaigns, Pricing, and Scheduling" is self-explanatory and at the discretion of the administrator and/or supervisors (see Figure 39). The administrator can set a specific budget for the advertising campaign that will be used efficiently by Facebook. This budget can be dollars per day or set as a lifetime budget. This allows for precise budgeting and scheduling of advertisements that the planning department can account for. It is suggested that administrators set a conservative budget initially to determine the effectiveness of the advertisements. Some cities have a lifetime budget of up to $\$ 250$ before reaching a plateau of fans. Therefore use advertisements to grow the fan base and reduce funding as soon as fan growth begins to slow.

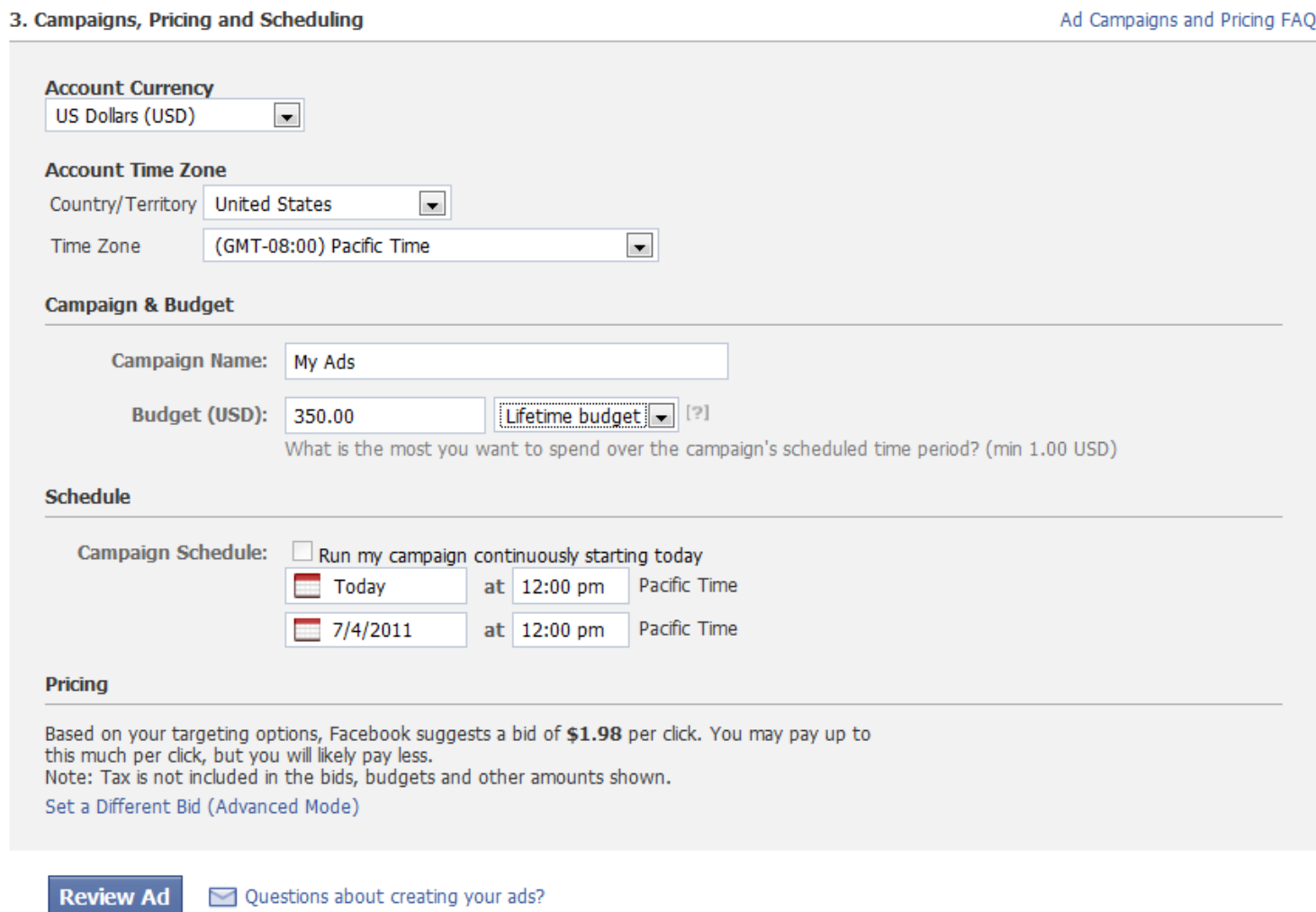

Review Ad $\square$ Questions about creating your ads? 
In review the following is essential for Advertising on Facebook:

- Set a budget and timeline for the advertising campaign. Ensure that the budget is used efficiently by targeting only the potential users who will become a fan of the Page.

- Determine the geographical reach of the advertising campaign. Essentially only city residents should be targeted to become a fan of the Page.

- Monitor and update the advertising campaign based on information/demographics of the Page. This requires the administrator to review the "Insight" section and change the targeting audience accordingly. An example could be if there is a disproportionate amount of 18-24 years olds on the site, then advertising may be targeted to "25-Any" age in the section.

- Create guidelines for marketing and using the Facebook service to ensure an efficient use of funds.

- Reevaluate the campaign when a plateau is reached. If fan growth begins to slow, then it may be necessary to reduce the advertising funding appropriately.

\section{Badges and Social Plugins}

Badges have recently been renamed to "Social Plugins" and for the purpose of this section will be used interchangeably. Badges are virtual portals to the Facebook Page that can be inserted into any website that uses HTML (which accounts for most websites). Badges can vary in size and relative information and provide a form of advertising for free when integrated with any website. cities can use social plugins in a variety of ways, but the most common is to add a "Like" button to the city or department website that when selected will take potential Facebook users directly to the Facebook Page. Other 
options for the administrator are including a live stream window within the city or department website. The City of Atascadero provides a dedicated page for social media and includes different Facebook page live streams. This social plugin provides information of the latest updates to the Facebook page without requiring users to actually visit the page (see Figure 40).
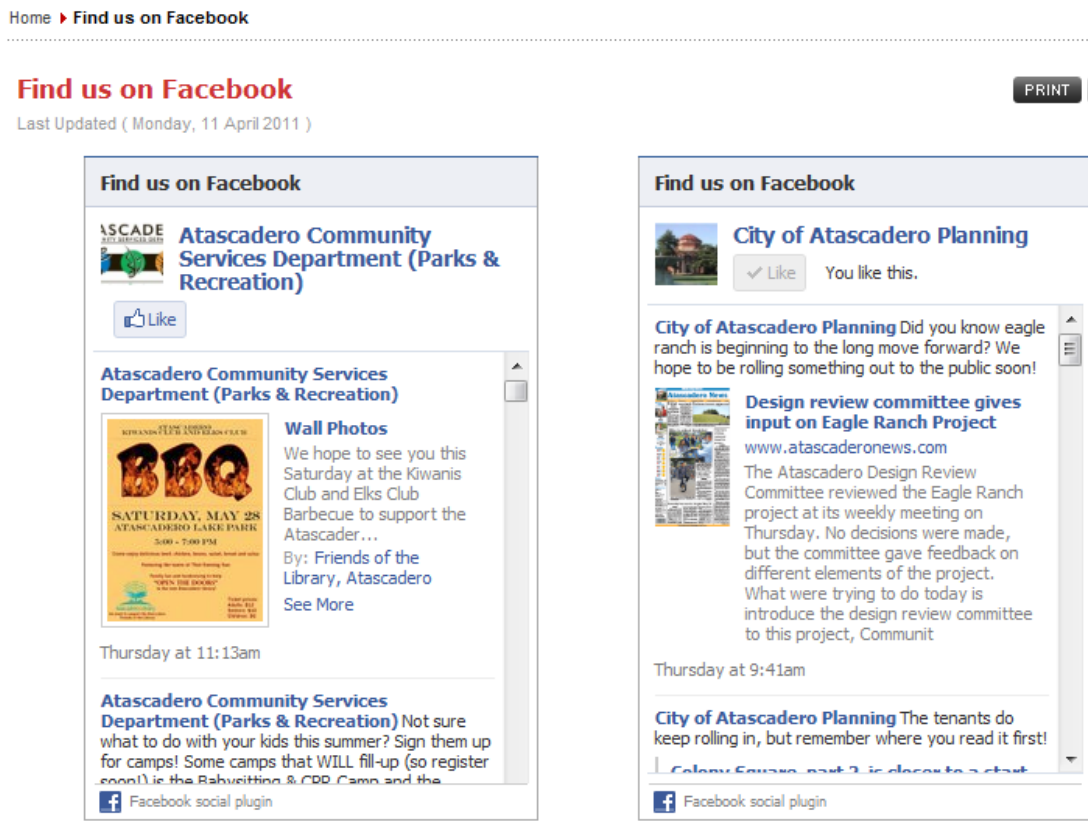

Login

Use your account to

sign up for mailing

lists and to track

contact requests

Interact

Contact Us

Find phone

numbers, websites,

or submit a request

online.

Public Records

Search Public

Records currently

available online

Report a Crime

Use our online tool

for reporting

non-emergency

crimes.

Figure 40 Social Plugins on the City of Atascadero Website

Badges and icons of the Facebook logo should be presented at the city home page;

alternatively badges/icons can be incorporated into the planning department homepage of the city. However potential fans may not visit the planning department or any other specific department and as such the main city homepage is a recommended area for badges and icons. A good example of this is the City of Long Beach, California, where the city homepage incorporates a dedicated social media section that is immediately identifiable (see Figure 41). Once this link is selected, a dedicated page that lists the various Facebook pages as well as other social media pages is presented. From here links 
can be selected (as opposed to a badge or icon) to take users directly to the Page of choice

(Figure 42).

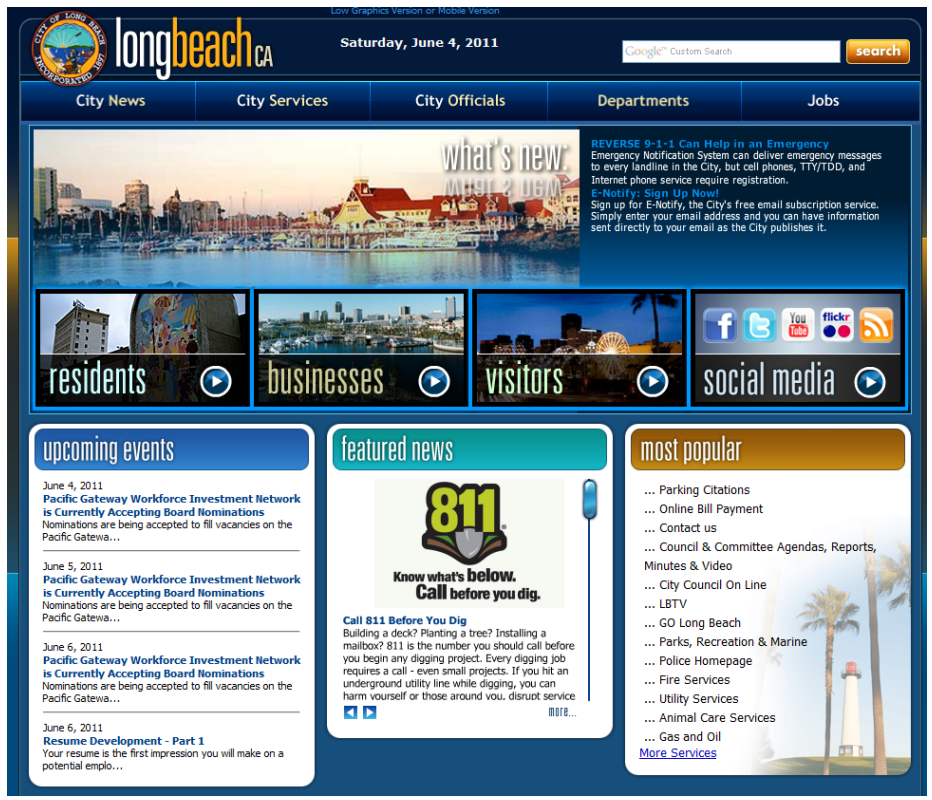

Figure 41 City of Long Beach Website Homepage with Clear and Identifiable Social Media Icons

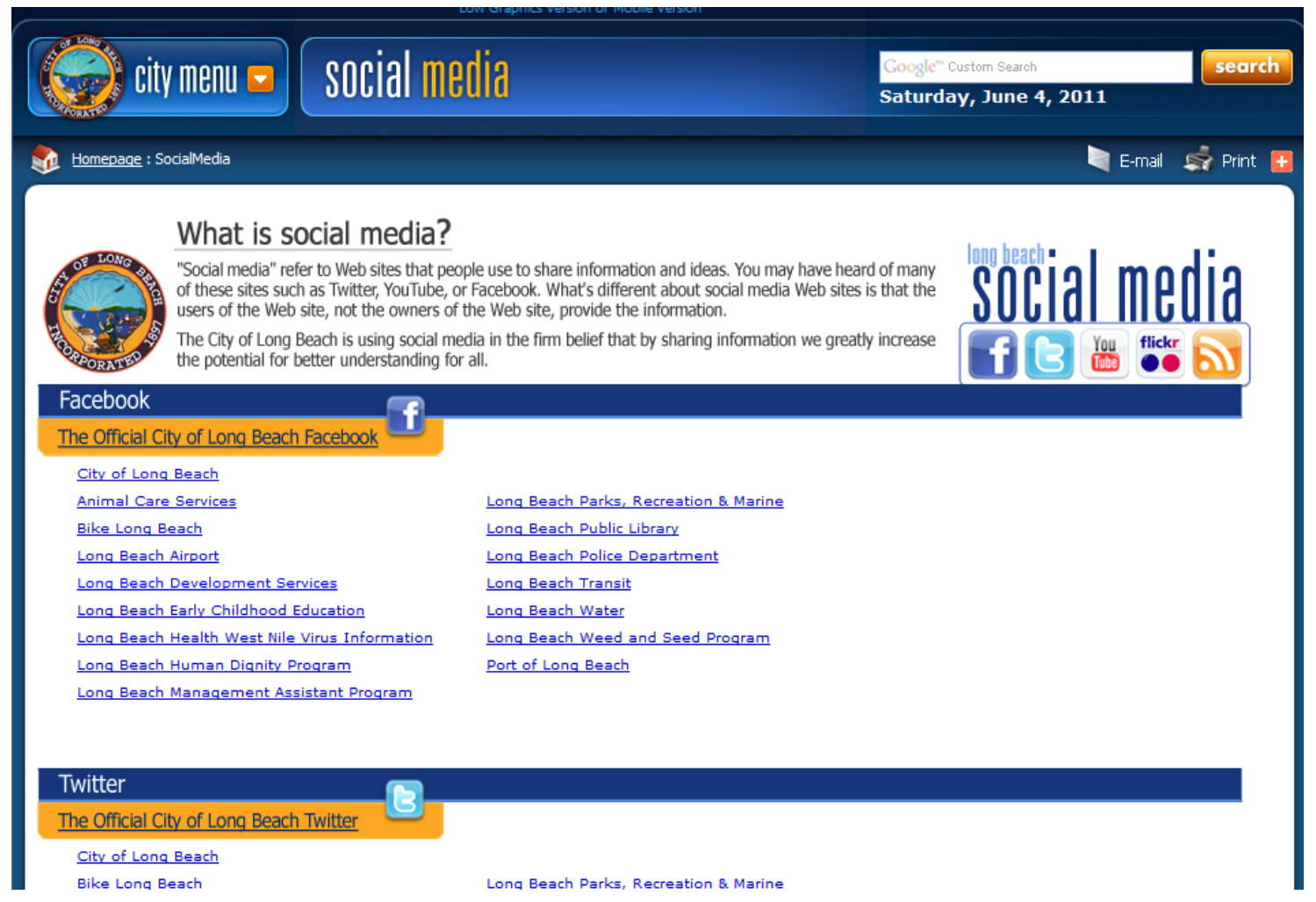

Figure 42 Lists of Social Media Pages Categorized by Media Type 
Badges, social plugins, icons, and links all provide free marketing from within the city or planning department webpage. However, the Information Technology department should be contacted to determine which types of social plugins will be appropriate and flow with the overall theme/look of the city webpage. Badges and links require the use of HTML code (created by Facebook) to simply be copied and pasted into the HTML code of the website.

In review the following is essential for Badges and Social Plugins:

- Understand what type of badge or social plugin is appropriate to use on the city website.

- Contact the responsible agency or department for inserting HTML code into the city website and determine how potential Facebook fans will view the badges.

- Badges and social plugins are a form of free advertising or acknowledgement of the department's Facebook Page. It is recommended that the planning department use a variety of social plugins to link the department's webpage with the Facebook Page. 


\section{BIBLIOGRAPHY}

Arnstein, S. R. . (1969). A ladder of citizen participation . Journal of the American Institute of Planners, 35(3), 216-224.

Atascadero, (2011, February 28). City of atascadero planning. Retrieved from http://www.facebook.com/planningatascadero.com

Berman, E. (2007). Dealing with cynical citizens. Public Adminstration Review, 57(2), 105-112.

Box, R.C. (1998). Citizen governance: leading america communities into the $21 \mathrm{st}$ century. Thousand Oaks, CA: Sage Publications

Brabham, D. (2009). Crowdsourcing the public participation process for planning projects. Planning Theory, 8, 242-262.

Brown, R. (1953). Ralph m. brown act. Retrieved from http://www.leginfo.ca.gov/

Burdi, L. (2003). An interactive web-based system in the urban design approval process: collabita. Informally published manuscript, Harvard Design School, Harvard, Cambridge, MA.

Coglianese, C. (1997). Assessing consensus: the promise and performance of negotiated rulemaking. Duke Law Journal, 46(6), 1255-1349.

David, J., Reischle, T., Carrick, R., \& Kartenich, C. (1997). Citizen participation lessons from a local united way planning process. Journal of the American Planning Association, 63(3), 345-355.

Davison, J. (2007). The virtual planning. exploring public participation planning through internet-based virtual reality systems. Informally published manuscript, The University of Calgary, Calgary, Canada.

Day, D. (1997). Citizen participation in the planning process . Journal of Planning Literature , 11(3), 421-434.

Deshler, D. and Sock., D. [1985]. Community Development Participation: A Concept Review of the International Literature. Paper presented at the International League for Social Commitment in Adult Education. Sweden.

Eliasoph, N. (1998). Avoiding politics: how americans produce apathy in everyday life. Cambridge: Cambridge University Press.

Evans, C. (2010). The new generation of public participation: internet-based participation tools. Planning practice + research, 25(3), 397-408. 
Facebook, (2011). Statistics. Retrieved from

http://www.facebook.com/press/info.php?statistics

Gordon, E. (2010). Community planit. Retrieved from http://engagementgamelab.org/

Govloop, (2011, January 17). How to: build an awesome government facebook page with tons of fans and engagement. Retrieved from http://www.govloop.com/

Harris, A., Wyn, J., \& Younes, S. (2010). Beyond apathetic or activist youth: 'ordinary' young people and contemporary forms of participation. Young, 18(1)(9-32),

Irvin, R., \& Stansbury, J. (2004). Citizen participation in decision making: is it worth the effort?. Public Administration Review, 64(1), 55-65

League of California cities. (2007). Open \& Public IV: A Guide to the Ralph M. Brown Act. California: League of California cities.

Laurian, L., \& Shaw, M.M. (2009). Evaluation of public participation: the practices of certified planner. Journal of Planning Education and Research, 28, 293-309.

Lee, J. (2009, July 31). Government entites follow americans' lead by boosting their digital presence. $P R$ Week, p. 14

Lewis, S., Pea, R., \& Rosen, J. (2010). Beyond participation to co-creation of meaning: mobile social media in generative learning communities. Social Science Information, 49(3), 351-369.

Lincoln, A. (1863). Gettysburg address. (2011). Retrieved from http://www.ourdocuments.gov/doc.php?flash=true $\& d o c=36$

Lyn, K., \& Martin, J.A. (1991). Enhancing citizen participation: panel designs, perspectives and policy formation. Journal of Policy Analysis and Management, 10(1), 46-63.

Oldfield, A. (1990). Citizenship and community: civic republicanism and the modern world. London: Routledge

Reed, M. (2001). Apathy and its discontents: social capital and social awkwardness in American life. New political science, 23(3),

Stivers, C. (1990). The public agency as polls: active citizenship in the administrative state. Administration and Society 22(1): 86-105

Portland Development Commission, Public Affairs Department, (2007). Public participation manual. Portland, Oregon : 
Putnam, R. (2000). Bowling alone: the collapse and revival of american community. New York: Simon and Schuster.

Pyle, G. (2010, October 12). Private vision. public oversight. Buffalo News,

Sanoff, H. (2000). Community Participation Methods in Design and Planning. New York: Wiley.

Tapia, A., \& Ortiz, J.A. (2010). Municipalities deploying wireless internet to increase civic engagement. Social Science Computer Review, 28(1), 93-117.

United States Census Bureau. (2010). Profile of general population and housing characteristics. Retrieved from http://factfinder2.census.gov

Waters, R., Burnett, E., Lamm, A., \& Lucas, J. (2009). Engaging stakeholders through social networking: how nonprofit organizations are using facebook. Public Relations Review, 35, 102-106.

Webler, T., Tuler, S., \& Krueger, R. (2001). What is a good public participation process? five perspectives from the public. Environmental Management, 27(3), 435-450.

Webler, T., \& Tuler, S. (2002). Unlocking the puzzle of public participation. Bulletin of Science Technology \& Society, 22(3), 179-189.

Webler, T., Tuler, S., Krueger, R., \& Lucas, J. (2009). Engaging stakeholders through social networking: how nonprofit organizations are using facebook. Public Relations Review, 34, 102-106.

Yin, Robert. [2003]. Case Study Research: Design and Methods, 3rd edition. United States: Sage Publications, Inc. 
Appendix A: City Interview Transcripts 


\section{City of Sterling Heights, Michigan}

\section{What is the primary goal of using Facebook (and social media in general) for the City of Pocatello?}

MISSION STATEMENT The Community Relations Department operates and populates social media sites as part of its overall mission of outreach to the Sterling Heights community. These new means of technology-based communication provide the City with yet another outlet for reaching Sterling Heights businesses and residents with vital information about their local government. At the same time, social media sites have an interactive feature that provides the City with constructive feedback that is invaluable when evaluating whether City services and programming are meeting their objectives.

\section{How did the city decide to use social media? Additionally, which department suggested the idea?}

The Community Relations Department launched the city's Facebook, You Tube and Twitter pages in fall, 2009. These social media outlets were, from the very beginning, intended to be used by the city in a manner consistent with our overall mission statement: "to provide the city with yet another outlet for reaching Sterling Heights businesses and residents with vital information about their local government."

\section{What is the process for running the page? Is there one person who is responsible or are there multiple admins? Additionally are there any security concerns with admin rights?}

Please see our adopted guidelines below...

To ensure that the Community Relations Department's social media sites function as intended and to prevent misuse, the following set of guidelines will be enforced:

1. Comments or other forms of feedback must be responsive to the postings by the Community Relations Department. All comments and/or feedback will be removed after being reviewed/evaluated and, if appropriate, referred to the appropriate City office or department for follow-up.

2. Comments or other forms of feedback containing personal attacks on persons or institutions or having profane, derogatory, discriminatory, hateful, threatening, unlawful, misleading, malicious or pornographic content shall be removed. The Community Relations Department's social media sites will not be used for purposes inconsistent with its stated mission.

3. Public employees, elected and appointed officials, and those seeking public office shall not use the social media sites to advance personal, financial, and/or political agendas.

4. Comments having links to sites that do not conform to these guidelines will be removed.

The Community Relations Department reserves the right to amend the foregoing guidelines and apply them to new forms of social media as circumstances dictate.

\section{Is there an internal "terms of use" protocol that those running the Facebook page must} follow?

\section{Same as above}


5. What have you found to be the main issues with the page? For example, are there concerns with the public's ability to comment on posts? Are there concerns with providing enough content to post on a regular basis? etc.

These guidelines are fair, impartial, and do not discriminate. They ensure the authenticity of posted information and prevent the city's social media outlets from being utilized for commercial, personal or political gain. The guidelines are in unison with other communication tools utilized by the city including our government access channels, web site, e-newsletters, electronic message signs, radio station, etc.

\section{Has the page obtained the goals the City set out to accomplish initially?}

Yes. We have more than 3,500 people following us on Facebook. Our Twitter page numbers are also growing. These social media sites give us greater access to a new demographic and users seem to be more responsive than those who receive e-mail list newsletters and other traditional communication mediums. 


\section{Tampa, Florida}

\section{What is the primary goal of using Facebook (and social media in general) for the City of Pocatello?}

It provides us with another means (tool) in which to connect with our residents and members of the public. It opens a two-way dialogue that traditional public relations programs should have, but often does not deliver. Most importantly, we use it to drive people back to our website - with over 7,000 pages of information - providing additional details and services online.

\section{How did the city decide to use social media? Additionally, which department suggested the idea?}

Currently, we have Facebook, Twitter and You Tube. Each was decided independently - based on what the return on our investment of time. The Public Affairs Office made the decision to create city accounts.

\section{What is the process for running the page? Is there one person who is responsible or are there multiple admins? Additionally are there any security concerns with admin rights?}

Originally, the was one person from Public Affairs responsible for each. Departments submitted entries to Public Affairs for posting. Recently, we provided members from our "high volume" departments direct access to post for themselves. These individuals already authorized to speak to the media and public on behalf of the city so there are no security concerns.

\section{Is there an internal "terms of use" protocol that those running the Facebook page must follow?}

The State Attorney issued an opinion that requires us to archive our postings. For Twitter and You Tube we have an rss reader that does it for us, however with Facebook, we take screen shots for every posting and save the file on a main drive that all approved users have access to. Additionally, they follow the messaging standards of the City of Tampa and Social Media Strategy (below).

\section{Social Media Strategy}

Social media, defined here as any website or online forum that allows for open communication, is changing the way we as a city government are able to interact with our residents and members of the public at large. Social media allows us to connect with people in a comfortable, convenient forum. It also helps to open the two-way dialogue traditional public relations programs require but often do not deliver. In order to establish a successful program, it must be integrated with the City's current communications program.

The City's social media participation will be handled through the Public Affairs Division in order to maintain the integrity of the City's overall communication strategies and programs. Public Affairs will establish accounts over various platforms of social communication in an incremental manner, but will limit participation in order to maintain the quality of the City's presence in the digital arena.

Each division and/or department should submit information and updates to Public Affairs for posting on the City's Twitter account and the City's Facebook page. Departments and divisions are encouraged to submit information of value to city residents and the general public including 
upcoming events, programs and projects. All submissions should attempt to encourage and direct the targeted audience to visit TampaGov for additional information. A link should be provided with the submission.

Though Public Affairs does not restrict the personal use of social media sites outside of the office, we encourage good judgment. Any time you write something on your personal account or participate in a blog and the information posted is associated with your department or division, we suggest that you be mindful that posts are public. You should consider using a disclaimer such as: "The postings on this site are my own and do not represent the City of Tampa's positions, strategies or opinions." Also, be mindful of your personal reputation as well.

\section{What have you found to be the main issues with the page? For example, are there concerns with the public's ability to comment on posts? Are there concerns with providing enough content to post on a regular basis? etc.}

We don't really have any issues - other than ensuring there is fresh content and the page doesn't just wither away. Our page was created as a fan page and people can post to the wall, which we do monitor. City postings are increasing and do occur on a more regular basis with the addition of the high volume department users having direct access for posting.

\section{Has the page obtained the goals the City set out to accomplish initially?}

Yes, we feel it does help drive people back to the City's website providing additional information. Additionally, it allows the public another avenue in which to communicate, share and find information about the City . 


\section{Yuba City, California}

\section{What is the primary goal of using Facebook (and social media in general) for the City of Pocatello?}

The primary goal is to disseminate information to the public and engage a younger population in government actions. Currently we send out post cards and newspaper articles.

\section{How did the city decide to use social media? Additionally, which department suggested the idea?}

The economic development department recommended the idea. The younger staff from public works also suggested the idea. These two departments joined together to develop and implement the Facebook page.

\section{What is the process for running the page? Is there one person who is responsible or are there multiple admins? Additionally are there any security concerns with admin rights?}

We currently have set our security to allow four administrators including myself in the economic development department. Public works, Parks and Recreation, and the City Clerk are the four department in charge of the page. Less than 20 minutes per day on average is spent updating and maintaining the page.

\section{Is there an internal "terms of use" protocol that those running the Facebook page must follow?}

There is no formal policy, instead we follow internal internet guidelines. There is a verbal agreement that, for example, all photos must include a brief title and description. We post daily or twice daily as a first come first serve policy for those wanting to post. Mainly newspaper articles are posted and the City is promoted in the best way possible.

\section{What have you found to be the main issues with the page? For example, are there concerns with the public's ability to comment on posts? Are there concerns with providing enough content to post on a regular basis? etc.}

We like to ensure regular quality content. Our population is only 65,000 people but we are the hub for the region. A possible issue is ensuring good dialogue between departments to get information for the page. We allow for the public to comment onn the page. We include a terms of use for the public to follow as well that includes the mission of the city. We do not allow profanity or any abuse on the page and so far we have not had any issues. We make sure not to respond editorially to comments and make sure we provide true and accurate information. We do not engage in a public discussion with fans. Additionally we ask any of the City Council members to never post on the page as it may constitute a meeting and violate the Brown Act.

\section{Has the page obtained the goals the City set out to accomplish initially?}

With regard to our original goals, yes. With limited staff time we have been just redirecting information via the status update. We have now created a Twitter page to augment the social 
media portion of the City. We have a final goal to connect and engage demographics of younger generations, which has not been accomplished as of yet and so we believe there is still room to grow. 


\section{Waco Texas}

1. What is the primary goal of using Facebook (and social media in general) for the City of Pocatello?

Our primary goal is to inform the public, as we do with all of our communications tools. The special thing about Facebook is that we can inform those that choose to 'follow' us and it's a free option to use.

\section{How did the city decide to use social media? Additionally, which department suggested the idea?}

Municipal Information and the Convention and Visitor's Bureau pushed the issue of starting the use of social media. There are different rules that apply to city government with Open Records Requests, etc. We decided to go forward when we evaluated the number of people we could reach for no cost.

3. What is the process for running the page? Is there one person who is responsible or are there multiple admins? Additionally are there any security concerns with admin rights?

The departments that manage their own Facebook pages (Public Information, Convention and Visitor's Bureau, Public Health District, Zoo, etc.) maintain them within their departments according to their own policies. In Municipal Information, we have three administrators but for the majority of the postings, they run through one person and the other two are just for back-up. Because our department is small, we don't worry too much about security issues but do police the comments left on the page.

\section{Is there an internal "terms of use" protocol that those running the Facebook page must follow?}

We don't have a policy specific to Facebook but employees to sign a policy specific to the use of social media outlining that they are responsible for their own posts and information is subject to open records requests.

5. What have you found to be the main issues with the page? For example, are there concerns with the public's ability to comment on posts? Are there concerns with providing enough content to post on a regular basis? etc.

We try to post something everyday- mostly information such as things we've sent out via press release or upcoming events. We haven't had any issues with ideas on what to post.

Sometimes comments are posted that need to be deleted but I monitor them via cell phone and can delete them if needed.

\section{Has the page obtained the goals the City set out to accomplish initially?}

Our page seems to grow consistently and gives us some direct feedback from our citizens. So, I would say that it does accomplish what we need it to do. And I think it's beneficial to explore all the 'free' options out there for communication with citizens. 


\section{City of Portland, Oregon}

\section{What is the primary goal of using Facebook (and social media in general) for the City of Portland?}

The page was created just for the pdx plan, not for the planning department. The primary goal was to reach the younger generations that are not typically a part of the process. Typically there are older, white, educated people with disposable time. This page was an attempt to broaden the demographic and reach a segment that is not represented. They recognize that this will only reach younger techno savvy people and perhaps not low income people and they recognize that social media is not a replacement tool for outreach.

\section{How did the city decide to use social media? Additionally, which department suggested the idea?}

The Mayor was responsible for the movement towards using social media. He merged the Planning department with Sustainability department in order to combine the services for efficiency. Suitability already had a Facebook page and so planning decided to use the resources for its own page.

\section{What is the process for running the page? Is there one person who is responsible or are there multiple admins? Additionally are there any security concerns with admin rights?}

Eden is responsible for the page and is the only one with administrative rights. She tweets twice a week at least and once a week on Facebook. There is a judgment call on whether to tweet or update about valuable information as the pdx plan is in 3 phases, information is not released all at once. She updates about events, information, and posts surveys for people to take via surveymonkey.com. She uses an editorial calendar to release information smoothly and not all at once in one day and not release any information another day.

\section{Is there an internal "terms of use" protocol that those running the Facebook page must follow?}

There is a citywide social media policy, however the person who championed it left for another city and so an outside web consultant was hired for the entire city and wrote the policy. The department has an interim policy based on internal practices that follows a nonprofits social policy. The issue at the moment is deciding who will be the media spokesperson for social media.

\section{What have you found to be the main issues with the page? For example, are there concerns with the public's ability to comment on posts? Are there concerns with providing enough content to post on a regular basis? etc.}

Commenting: there are actually few comments left on the pdx page where most have been positive and/or inquires about the plan. These questions are responded to via direct messaging and not on the wall by the City. Twitter on the other hand is where they receive nasty tweets and have been criticized for not following twitter etiquette.

\section{Has the page obtained the goals the City set out to accomplish initially?}


They measure success through staff capacity and keeping the attention of the followers/friends of each site. There are ebbs and flows with regard to viewership and content. Success is also measured by the respondents of the surveys they post on the page, where they have been seeing an increase in online responses. 


\section{Savannah, Georgia}

\section{What is the primary goal of using Facebook (and social media in general) for the City of Pocatello?}

I think the saying is "fish where the fish are." We want to reach as many citizens as we can and social media is just hugely popular across so many demographics. It hit a point where the opportunity outweighed the perceived risks. We want folks to find it easy to connect with their local government, we want to be accessible. Our goal with social media was to extend the reach of our messages, and we wanted to be open and participatory in that effort.

\section{How did the city decide to use social media? Additionally, which department suggested the idea?}

The Public Information Office led the effort, as we are the City's primary messenger, but we did it with a lot of support from our Cultural Affairs Department and other city staff who were excited about the possibilities presented by social media. Our office connected with other municipalities who were already into social media, we used these examples to develop our own strategy.

\section{What is the process for running the page? Is there one person who is responsible or are there multiple admins? Additionally are there any security concerns with admin rights?}

The Public Information Office is the primary "keeper" of our social media accounts. We do have additional admins from our Fire and Police departments because we use the accounts during our St. Patrick's Day Festival (one of the largest in the country) to send out updates around the clock, and our office splits those shifts with our public safety folks. We also have an admin from our Cultural Affairs Department because they have regular updates and were champions of the idea from the start, and we wanted a centralized but comprehensive City of Savannah presence (as opposed to splitting the potential audience between multiple department or program accounts). As for security, we stress to our admins how careful they must be with their accounts and their passwords, and we ask them to regularly change their password, as they do with their internal network accounts.

\section{Is there an internal "terms of use" protocol that those running the Facebook page must follow?}

We do have a policy about what we post, how we handle comments and what sort of "voice" we'd like to see admins using. The basic policy is to use the social media sites for our news releases, and the major public events we host or sponsor. We delete comments that have profanity, slurs or other inappropriate language. We also delete posts that are not related to City business, or events and programs we sponsor. In cases where people have questions, we do our best to answer them or route them to the appropriate contacts. If someone is critical of the City or just wants to air an opinion about the City, we will address it if possible, if there is no official response or position from the City on the issue we will just let it stand. From the beginning it was important to us keep the "social" in social media, so we had to be open to comments and feedback, good or bad. 


\section{What have you found to be the main issues with the page? For example, are there concerns}

with the public's ability to comment on posts? Are there concerns with providing enough content to post on a regular basis? etc.

We did have concerns about comments initially, not knowing what to expect. But our experience has been overwhelmingly positive. We get critical comments occasionally, but by and large people are following the accounts because they are genuinely interested in what's going on and they appreciate what we're sharing. So far, we haven't had any issue with posting on a regular basis, but we are looking at ways to extend what we do on Facebook and Twitter. We use Twitter in a very minimal way right now, it's just tied to our Facebook account and automatically posts whatever we put on Facebook. l'd like to see us get more into the Twitter world, and we may in the future. The only concern at this point is keeping up with it. We have a small staff and multiple projects going on all the time, and we don't want anything to get lost in the shuffle, so we're thoughtfully exploring how we can sustain a bigger effort on the social media front.

\section{Has the page obtained the goals the City set out to accomplish initially?}

We intentionally and cautiously started small, so absolutely we've met and even exceeded our initial goals. We've got an active and growing audience. We're getting more feedback and participation. With such a positive base to work from, I expect we'll continue to do more with social media. Traditional communications efforts aren't going away, but I think social media and technology in general (mobile apps, interactive websites, etc.) are proving to be more of a "must have" than a "would be nice" toolset for government. 


\section{Anonymous City, Nebraska}

As I mentioned before, our social media accounts are relatively new - there are only a couple of accounts that have been around for more than a year. The City only recently opened up the firewall to allow employee access to Facebook and Twitter, so as you can imagine, we are still catching up.

\section{What is the primary goal of using Facebook (and social media in general) for the City?}

The City basically got an "official" Facebook page to garner attention for our application to the Google Fiber for Communities Program last March. The page and its maintenance were handed off to an outside company, Thought District - a "brand \& sales strategy company" - in order to leverage their experience with promotion and marketing in a short amount of time. I think they managed to get the page around 25,000 "likes" in about a month. I have no idea what they did, but it worked.

The City's Facebook page has since remained in their hands. We don't really have an overarching goal right now, and we haven't developed any concrete policy, so I think we're a little hesitant to take it over. This is just regarding the general City Facebook page though, and not individual departments.

Our division - Citizen Information Center - uses Twitter to send out notifications for news releases that are issued from the Mayor's Office, and for snow warnings (plowing operations, road closures, parking bans, etc.). Our goal with Twitter is to get information out to people via SMS (for free), and to supplement our email and RSS subscribers.

\section{How did the city decide to use social media? Additionally, which department suggested the idea?}

Again, Facebook for the Google Fiber thing; it was suggested by some Technology Committee or something. I decided to try Twitter for news releases to see if I could beat our email and RSS subscriber numbers.

3. What is the process for running the page? Is there one person who is responsible or are there multiple admins? Additionally are there any security concerns with admin rights?

City Facebook = all run by Thought District, so I have no idea what they do. I imagine they've got an intern somewhere doing it. Each city department runs their own pages, so there are a number of accounts with an equal number of admins. No real security concerns, I suppose.

4. Is there an internal "terms of use" protocol that those running the Facebook page must follow?

$<$ Left Blank>

5. What have you found to be the main issues with the page? For example, are there concerns with the public's ability to comment on posts? Are there concerns with providing enough content to post on a regular basis? etc. 
It appears you're much more interested in Facebook than anything else - I've forwarded your questions to someone at Thought District, so hopefully they can offer you something. Regarding Twitter so far, we've decided to not respond to @replies - mostly because they tend to be angry, rhetorical questions or comments about streets being closed, getting a parking ticket, potholes, etc. If there was a direct question, I would respond, but that hasn't happened. No interaction is perhaps risky, and may make the account appear automated (it is not), but we don't have the staff to monitor and respond. That's also a big reason why we aren't in direct control of our Facebook page. We also aren't sure what our content would be on the Facebook page - the fluffy question style that Thought District employs works, but we also don't have the staff to come up with this. I guess we need more marketing interns.

\section{Has the page obtained the goals the City set out to accomplish initially?}

Well, we didn't get the Google Fiber thing. Since then, my bosses have pretty much ignored our Facebook page and have let Thought District do their thing. I don't know the specifics of the agreement we have with them. With Twitter, we've surpassed our email subscription list numbers, and see a bit of activity from followers. [This] is a small town and has a small \#LNK following, but they're loyal and the numbers are slowly growing. We haven't promoted it at all yet, save for a few links here and there. 


\section{Fargo, North Dakota}

1. What is the primary goal of using Facebook (and social media in general) for the City of Pocatello?

To share information quickly and directly with residents.

2. How did the city decide to use social media? Additionally, which department suggested the idea?

The City of Fargo began using social media primarily to share information with residents instantaneously during emergency situations, such as spring flooding. In the past, we had been relying on the media to share the bulk of our information, however information as not always relayed quickly enough or accurately. The idea stemmed from the communications office, however other departments has been dabbling with Facebook before that.

3. What is the process for running the page? Is there one person who is responsible or are there multiple admins? Additionally are there any security concerns with admin rights?

The communications manager runs the official City of Fargo Facebook page, however some of the larger City departments have their own pages as well (Fire, Police, Library, Health). The communications manager maintains admin rights to all departmental social media sites, however a dept. staff person oversees daily messaging and site maintenance.

4. Is there an internal "terms of use" protocol that those running the Facebook page must follow?

Yes

5. What have you found to be the main issues with the page? For example, are there concerns with the public's ability to comment on posts? Are there concerns with providing enough content to post on a regular basis? etc.

Our biggest concern is updating the site often enough to keep residents interested. It can be very time consuming to post pictures, etc. on a regular basis.

6. Has the page obtained the goals the City set out to accomplish initially?

Yes, however our number of friends is still low. Twitter is far more popular with our residents. 


\section{Cincinnati, Ohio}

The City of Cincinnati Department of City Planning and Buildings has two Facebook pages that we have used in the past couple of years and we will likely integrate a third sometime in the next few months.

The first page we released was one called Cincinnati Counts which was focused on getting out the word about the 2010 census. We have not posted to it since the census was complete, but we will probably post one or two more times before we completely retire it. The 2010 data is coming out gradually, and once we have it all analyzed we will probably sent out a message telling people where they can view the data online.

The second page we released is one for Plan Cincinnati, the City's comprehensive plan, which is currently in process.

The third page we might create is for a process related to a $\$ 2.4$ million Community Challenge Planning grant we received from HUD earlier this year. We will be going through a 3-year process to completely re-write and integrate our zoning, building, and subdivision codes.

\section{What is the primary goal of using Facebook (and social media in general) for the City of Pocatello?}

Facebook is simply another method of communicating with the public and providing notice of meetings or other events associated with some of our larger projects. With day-to-day work as well as large projects, the primary method of communicating is still email blasts and direct mailings. Email blasts are still an effective way for us to spread the word about something to a large group of people. Also, for our regulatory work we are required to provide direct written notice to property owners.

\section{How did the city decide to use social media? Additionally, which department suggested the idea?}

I don't know specifically who in Planning suggested using Facebook for the Census and Plan Cincinnati. Probably one of us who was using Facebook in our personal life.

\section{What is the process for running the page? Is there one person who is responsible or are there multiple admins? Additionally are there any security concerns with admin rights?}

There are multiple admins, but there are probably only about two - four of us who actually post things. This has fluctuated over time, depending on staff. There have not been any security concerns, but we have some staff who are not always detail-oriented when it comes to spelling and grammar when using social media. I know it is common to use texting shorthand on personal FB pages, but it is completely unacceptable on a page sponsored by a City department. If something is incorrect I have had to delete it and re-post.

\section{Is there an internal "terms of use" protocol that those running the Facebook page must follow?}


If someone wants to post something on the page they need to run the language through me first. Sometimes that happens, sometimes it doesn't. (See above)

\section{What have you found to be the main issues with the page? For example, are there concerns with the public's ability to comment on posts? Are there concerns with providing enough content to post on a regular basis? etc.}

Early on, our primary issue was that we could not access Facebook from work, so we were trying to do updates from our homes on personal time, which was very difficult to manage. A couple of months ago we finally received access on our work computers.

We do have ongoing concerns about the public posting on the site. I have removed most of the spam (people selling things) but since we have never had a real policy, anyone from the public can post any comment about anything. Sometimes the comments are very critical or to the other extreme: not related to Comprehensive Planning. Also, the way that planning processes are generally structured, we don't always have something to say. When we are advertising meetings or have upcoming events we have a lot of things to post. However, there are large stretches where we don't post anything at all because we are doing research or writing and there really isn't anything to report. We know there are things we can and should be posting during that time to keep the project on the radar, but quite honestly we are already pretty stretched for both time and staff, and there is always something else that takes precedence.

\section{Has the page obtained the goals the City set out to accomplish initially?}

Yes and no - we do appreciate being able to use FB as one of our many tools for increasing public awareness about projects and related events, but we know we have not been able to tap into its true potential. 


\section{Eugene Oregon}

\section{What is the primary goal of using Facebook (and social media in general) for the City of Pocatello?}

The City of Eugene views social media as another tool to help engage and inform community members and others. It often provides a way to reach people who may not typically be involved with City activities. In the Library, Recreation and Cultural Services Dept. (LRCS) for instance, FB provides a direct link with our patrons and segments the audience so that the Aquatics FB has different fans than the Outdoor Program FB, for example.

Ultimately, we hope social media moves us forward on our larger City goals to provide more transparent and interactive communication and to offer public engagement that involves the community broadly.

\section{How did the city decide to use social media? Additionally, which department suggested the idea?}

The use of social media by City programs began fairly organically. It is hard to pinpoint which department or program first began using social media, but our Recreation programs have been utilizing it for several years. Many programs and projects have established Facebook pages to communicate with participants. Currently, Public Works construction, the airport, Police, Downtown Development, our performing arts center, Recreation Services, and the public library also use Twitter. The City (as a whole) just recently created a Facebook page.

\section{What is the process for running the page? Is there one person who is responsible or are there multiple admins? Additionally are there any security concerns with admin rights?}

The programs that establish the social media accounts are responsible for running them. In LRCS, staff are assigned to populate its FB pages. In addition, two staff are administrators for all Recreation pages to provide additional oversight.

The City's general Facebook page is available for members of our Public Information Team to post to. It has one admin login that is shared with team members so they can log into the account and post information. We have not run into any security concerns at this point.

\section{Is there an internal "terms of use" protocol that those running the Facebook page must follow?}

Since social media is continually evolving, the Library Recreation \& Cultural Services Department developed a "risk analysis" for determining whether to establish a social media site. This document also includes "guidelines" that include a procedure and naming policy (attached). LRCS also adapted established policies to take in social media, such as the photo policy (included on attached).

5. What have you found to be the main issues with the page? For example, are there concerns with the public's ability to comment on posts? Are there concerns with providing enough content to post on a regular basis? etc. 
Concerns vary based on the project involved. In general, there are some concerns about the general return on investment of staff time. I'm not aware of concerns about the public's ability to post. We do remove posts that are not relevant to City business - i.e. ads or spam. Yes, keeping our City Facebook page up to date and fresh is an ongoing concern.

\section{Has the page obtained the goals the City set out to accomplish initially?}

Since the City's general page is relatively new it seems too early to tell if it has achieved our goals. 


\section{Ann Arbor, Michigan}

\section{What is the primary goal of using Facebook (and social media in general) for the City of Pocatello?}

Unlike the City's website, which is static, social media tools offer the city the opportunity to interact with citizens in a two-way conversation in real time using a relatively inexpensive tool. In addition, followers or fans can be easily tracked and have shown an interest in receiving our content, which is pushed to them.

\section{How did the city decide to use social media? Additionally, which department suggested the idea?}

The Communications Office took the lead on social media usage due to the fact that these tools were quickly becoming main stream as well as the fact that Ann Arbor is a very tech-savvy town and the ideal place to try out new modes of communication. In addition, the city's newspaper is online and no longer printed daily adding to our populations reliance on online media.

\section{What is the process for running the page? Is there one person who is responsible or are there multiple admins? Additionally are there any security concerns with admin rights?}

I manage the official City of Ann Arbor Facebook and Twitter accounts, but the city does have approximately five other Facebook and Twitter sites for individual business units, such as CTN, Parks and Recreation, Energy, etc. Going forward new FB or Twitter sites must be approved via the Communications Office, which is outlined in our Administrative Policy and Procedures. To date, we have not had any reason to be concerned with administrator right usage by approved staff.

\section{Is there an internal "terms of use" protocol that those running the Facebook page must follow?}

Yes, APP 201 (attached).

\section{What have you found to be the main issues with the page? For example, are there concerns with the public's ability to comment on posts? Are there concerns with providing enough content to post on a regular basis? etc.}

The main issues with the City's Facebook and Twitter pages is having the time to populate several times a day. While we have a ton of content we could promote throughout the day, I do not have the time to do so. Typically what happens is I update several times a week vs. hourly which isn't recommended as a best practice but is all I have time for. The only content I have had to remove to date has been maybe a handful of ads posted to the site for services. All other posts have added value to the conversation or identified an issue that the City needs to address - which was the goal of using this tool-real-time, interaction with citizens

\section{Has the page obtained the goals the City set out to accomplish initially?}

Since its creation the site has added followers each and every week and continues to gain. In addition, during two emergencies (gas line breaks at City Hall and City Hall evacuation) the FB 
site has allowed me to quickly update information away from City Hall and readily identify any issues that need to be resolved surrounding a specific situation. For instance, during this closure our customer service phone was not routing correctly and we were able to quickly fix this issue. In addition, followers can post specific questions about service updates or news items and I can answer on the wall so that others can benefit from the information. We also can track "likes" and better determine what services or information are resonating in the community. All of this is not possible with our website, even though we do utilize Google Analytics, the measurement tool is not as specific. 


\section{Anaheim, California}

1. What is the primary goal of using Facebook (and social media in general) for the City of Pocatello?

Brand and promote the City of Anaheim. Engage our residents.

\section{How did the city decide to use social media? Additionally, which department suggested the idea?}

The City Manager's Office suggested and implemented the idea. The City prides itself in using technology to encourage residents to reach City Hall, this is just another application that we decided to use to achieve that goal.

3. What is the process for running the page? Is there one person who is responsible or are there multiple admins? Additionally are there any security concerns with admin rights?

There are two admins that run the page, there is primarily one staff member from the External Affairs division that updates the page. When that staff member is out of the office or needs assistance, the second staff member that created the policy and established the Facebook account makes updates.

There aren't any concerns with admin rights.

4. Is there an internal "terms of use" protocol that those running the Facebook page must follow?

Yes, there is an internal policy that was established. Any department that is interested in setting up an account meets with the staff member who oversees the policy and goes over the best practices.

5. What have you found to be the main issues with the page? For example, are there concerns with the public's ability to comment on posts? Are there concerns with providing enough content to post on a regular basis? etc.

We have been very lucky and haven't experienced any problems. We don't allow the public to openly post to our page, rather they can post comments in respond to one of our posts. That has worked very well.

We usually have enough content to post, the challenge is ensuring it is comprehensive and represents all departments.

\section{Has the page obtained the goals the City set out to accomplish initially?}

Yes. While we strive to reach more people and increase our fan base, social media has been a great touch to reach our residents who may not otherwise engage in City news and events. 


\section{City of Pocatello, Idaho}

\section{What is the primary goal of using Facebook (and social media in general) for the City of Pocatello?}

My goal from the outset was to use Facebook (in particular) to connect with the community of local Pocatello internet users that might be less likely to visit our official city website in order to inform them about official city news. Pocatello is a relatively small city of 55,000 and a lot of communication takes place word of mouth. It was/is important to me that city news and information gets distributed to as many people as possible directly from the source. Distributing news and info via Facebook is somewhat less intrusive than and email because of the way the Facebook newsfeed works and efforts at developing a citizen email distribution list proved less than successful. Facebook users have the expectation that they will see information from the Facebook pages they are fans of, kind of goes with becoming a fan. With a traditional website you depend on web your audience to come to you and that can be a difficult task especially if you are trying to engage people with the daily business of local government (it's not that exciting). But with social media or a Facebook page you invest time to initially attract people to become a part of your network or a fan (which is a pretty minimal cost) and then because of network dynamics in no time at all you are distributing your news to a significant audience that may have taken you twice as long to develop otherwise. We established our Facebook page last year April 2010 and currently we have about 2000 followers and I would like to double that in the next year. As far as other social media go, I use Blip.tv to distribute video content. The goal here was mainly to have a facility to stream video of City Council meetings and other city specific content produced by the city public access TV station. Having Blip.tv as a tool has proved highly effective and cost-efficient. In the last two years I have posted over 200 videos that have been viewed/shared several thousands of times.

\section{How did the city decide to use social media? Additionally, which department suggested the idea?}

I have been in charge of web communication for almost three years. When I came on board at the city of Pocatello, it's web communication was severely lacking. I had to completely overhaul the city website and in doing so it was important for me to incorporate more innovative technologies to interact with our web audience. I also wrote my master's thesis on Facebook and have studied new media for several years, so it was a no brainer for me to setup a city Facebook page. It just took initiative on my part to set up the Facebook page and develop it in order to win other city administrators over on the idea - but I had no impediments - largely because what I do is a bit foreign to most city administrators. Also because the City of Pocatello does not have a Communication/Press department I was able to create a social media role for myself. I also use Blip.tv to stream and share city video content and I have setup a Twitter account but my challenge is how best to deploy Twitter right now, so currently it is dormant.

\section{What is the process for running the page? Is there one person who is responsible or are there multiple admins? Additionally are there any security concerns with admin rights?}

Currently, I am the only administrator. There are no significant security concerns since I am in charge of all City web content. As an aside, in fact currently the IT department firewall blocks Facebook for internal use by city staff. So I am the only user within the city that can bypass the city firewall to manage our Facebook page. In terms of daily management I post probably 2-3 items a week, and that largely depends on what is happening specifically at City Hall or with any 
of the 25 city departments. I select a news item to post or link to a highlight from the city's website. Not all city news is mirrored on the Facebook page so I do my best to make sure what is posted in informative, timely, and helpful to our community audience.

\section{Is there an internal "terms of use" protocol that those running the Facebook page must follow?}

No there is no internal "terms of use" protocol. The city's internal policy mechanisms still don't know how exactly to deal with new (social) media, so I manage the process with great flexibility and a little restraint so as not to push things too far. I plan on developing a protocol for use to ensure that the city continues to use Facebook as a means to communicate with the community into the future.

\section{What have you found to be the main issues with the page? For example, are there concerns with the public's ability to comment on posts? Are there concerns with providing enough content to post on a regular basis? etc.}

No real issues with the Facebook page per se. Yes, we get the occasional post from a citizen that is upset about some issue, but that is par for the course at any level of government. I have a policy of removing any posts that include obscenities or are complete rants. If it is a genuine criticism on how the city is managing an issue and others can learn something from the post or involves a debatable issue, then I encourage wall interactivity. However most of the interactivity that takes place on the city Facebook page involves my posts and the Facebook like button. In general the majority of users following the city do not post comments but use the like button as a means of communication. I also delete spam posts form individuals trying to advertise or market products or non-city related events.

\section{Has the page obtained the goals the City set out to accomplish initially?}

My main goal as I mentioned was to be able to connect with the community that may not have been paying attention to city news. I believe that I achieved that goal and now I have 2000 people who I am able to distribute city news to daily as part of their Facebook experience. I also think that I have justified the use of social media as an important tool in the business of local government. I am able to quickly disseminate city news to a broader spectrum of the community and that can be very beneficial, especially when it comes to city policies \& ordinances and in particular Fire, Police and Street alerts. 


\section{North Charleston, South Carolina}

\section{What is the primary goal of using Facebook (and social media in general) for the City of Pocatello?}

North Charleston uses a lot of social media including Twitter, YouTube, Blogs, and live streams. The main goal of using social media is to increase dispersal of information to the public as well as get feedback from the public. Also it is used to reach a different audience, ones that don't go to public meetings. Twitter sums it up well, 140 character tweets vs. 140 page council package. This condensed information reaches a younger generation. Also image and video works well for the younger generation.

\section{How did the city decide to use social media? Additionally, which department suggested the idea?}

I started the page and knew the intrinsic value of social media. I went to the mayor for approval which was a simple process. Social media is free and signing up is quick and so was an easy choice for the city to adopt it. Besides cities are already used to creating press releases as well as releasing photos, these can be looked at as blogs and Facebook photos respectively.

3. What is the process for running the page? Is there one person who is responsible or are there multiple admins? Additionally are there any security concerns with admin rights?

There are multiple admins only as backup. Most of the Facebook feed comes from the twitter account, yet we don't post to Facebook in the same volume as twitter. Only really important information is posted on Facebook. As far as security, the person conducting social media should be trusted and at an executive level. It is the same as going on the TV camera, each message posted is representational of the City - it's a political message.

\section{Is there an internal "terms of use" protocol that those running the Facebook page must follow?}

I manage the accounts and use my own discretion. So far there have been no issues from content. Negative messages are left and only explicit content is removed.

\section{What have you found to be the main issues with the page? For example, are there concerns with the public's ability to comment on posts? Are there concerns with providing enough content to post on a regular basis? etc.}

There are not a lot of explicit content posted but the page is monitored on a daily basis to filter anything explicit if necessary. We use TweetDeck as a platform to monitor our social media sites.

\section{Has the page obtained the goals the City set out to accomplish initially?}

Yes - we accomplished all of our goals named in question 1. People get wrapped up in the number of followers vs. the information to citizens. We managed to achieve the goals for free except for a $\$ 20$ fee for hard drive space with Google docs. We do not use the advertising on Facebook and still have managed to get a lot of followers. 
Appendix B: cities Contacted for Interviews 


\begin{tabular}{|c|c|c|c|c|c|}
\hline & City & Contact Date & Facebook Page & Notes & Follow Up \\
\hline 1 & Alexandria, Louisiana & $5 / 23 / 2011$ & $\begin{array}{l}\text { http://www.facebook.com/pages/City-of-Alexandria- } \\
\text { Louisiana/203149099696454?sk=wall }\end{array}$ & & \\
\hline 2 & Ames, lowa & $5 / 23 / 2011$ & http://www.facebook.com/cityofames & & \\
\hline 3 & Anaheim, California & $5 / 25 / 2011$ & http://www.facebook.com/cityofanaheim & $\begin{array}{l}\text { Sent ques tions on } \\
5 / 25 / 11\end{array}$ & RECEIVED ANSWERS \\
\hline 4 & Ann Arbor, Michigan & $5 / 23 / 2011$ & http://www.facebook.com/TheCityOfAnnArbor & $\begin{array}{l}\text { Sent questions on } \\
5 / 24 / 11\end{array}$ & RECEIVED ANSWERS \\
\hline 5 & Athens, Georgia & $5 / 24 / 2011$ & http://www.facebook.com/accgov?ref=mf & $\begin{array}{l}\text { Sent questions on } \\
5 / 24 / 11\end{array}$ & \\
\hline 6 & Auburn, Maine & $5 / 25 / 2011$ & $\begin{array}{l}\text { http://www.facebook.com/pages/City-of-Auburn-Maine- } \\
\text { Official/207249979309618?sk=page getting started }\end{array}$ & & \\
\hline 7 & Augusta, Georgia & $5 / 24 / 2011$ & $\begin{array}{l}\text { http://www.facebook.com/pages/City-of-Augusta- } \\
\text { Georgia/142356927602?v=box 3\&ref=sgm }\end{array}$ & & \\
\hline 8 & Aurora, Colorado & $3 / 8 / 2011$ & http://www.facebook.com/auroragov & & \\
\hline 9 & Austin, Texas & $3 / 8 / 2011$ & http://www.facebook.com/austintexas gov?ref=ts & $\begin{array}{l}\text { Sent questions on } \\
3 / 17 / 11 /\end{array}$ & $\begin{array}{l}\text { Follow up on email } \\
4 / 25 / 11\end{array}$ \\
\hline 10 & Baltimore, Maryland & $5 / 25 / 2011$ & $\begin{array}{l}\text { http://www.facebook.com/pages/Baltimore-Citv-2010- } \\
\text { Census-Complete-Count-Campaign/222498280367 }\end{array}$ & & \\
\hline 11 & Bangor, Maine & $5 / 25 / 2011$ & $\begin{array}{l}\text { http://www.facebook.com/pages/City-of-Bangor- } \\
\text { Maine/90511759973?ref=ts }\end{array}$ & & \\
\hline 12 & Bellevue, Nebraska & $3 / 8 / 2011$ & $\begin{array}{l}\text { http://www.facebook.com/group.php?gid=5372095149 } \\
\text { 2\#!!/group.php?gid=53720951492\&v=wall }\end{array}$ & $\begin{array}{l}\text { This is a group FB } \\
\text { page, not a Page }\end{array}$ & \\
\hline 13 & Billings, Montana & $3 / 8 / 2011$ & Link is broken & $\begin{array}{l}\text { Alerted them that } \\
\text { the main webpage } \\
\text { link is broken }\end{array}$ & \\
\hline 14 & Birmingham, Alabama & $5 / 23 / 2011$ & http://www.facebook.com/birmingham.alabama & $\begin{array}{l}\text { Sent Questions to } \\
\text { on } 5 / 23 / 11\end{array}$ & \\
\hline 15 & $\begin{array}{l}\text { Bismarck North } \\
\text { Dakota }\end{array}$ & $3 / 8 / 2011$ & $\begin{array}{l}\text { http://www.facebook.com/pages/City-of-Bis marck- } \\
\text { North-Dakota/103403749697601?v=wall }\end{array}$ & & \\
\hline 16 & Boise, Idaho & $3 / 8 / 2011$ & $\begin{array}{l}\text { http://www.facebook.com/pages/City-of- } \\
\text { Boise/102597609787637 }\end{array}$ & & \\
\hline 17 & \begin{tabular}{|l} 
Boston, \\
Massachusetts \\
\end{tabular} & $5 / 25 / 2011$ & http://www.facebook.com/cityofboston & & \\
\hline 18 & Boulder, Colorado & $3 / 8 / 2011$ & http://www.facebook.com/bouldercolorado.gov & & \\
\hline 19 & $\begin{array}{l}\text { Bowling Green, } \\
\text { Kentucky }\end{array}$ & $5 / 23 / 2011$ & $\begin{array}{l}\text { http://www.facebook.com/pages/City-of-Bowling-Green- } \\
\text { KY/54145957542 }\end{array}$ & & \\
\hline 20 & Cheyenne, Wyoming & $3 / 8 / 2011$ & $\begin{array}{l}\text { http://www.facebook.com/CityOfCheyenneCityWyoming } \\
\text { ?v=box } 3\end{array}$ & & \\
\hline 21 & Cincinnati, Ohio & $5 / 23 / 2011$ & $\begin{array}{l}\text { http://www.facebook.com/CincinnatiCommunityDevelo } \\
\text { pment }\end{array}$ & $\begin{array}{l}\text { Multiple } \\
\text { department } \\
\text { Facebook pages }\end{array}$ & RECEIVED ANSWERS \\
\hline 22 & $\begin{array}{l}\text { City of Saint Paul, } \\
\text { Minnesota }\end{array}$ & $5 / 10 / 2011$ & $\begin{array}{l}\text { http://www.facebook.com/pages/City-of-Saint- } \\
\text { Paul/79640426150?ref=ts\#!/pages/City-of-Saint- } \\
\text { Paul/79640426150?sk=wall }\end{array}$ & & \\
\hline 23 & $\begin{array}{l}\text { Colorado Springs, } \\
\text { Colorado }\end{array}$ & $3 / 8 / 2011$ & $\begin{array}{l}\text { http://www.facebook.com/pages/City-of-Colorado- } \\
\text { Springs/81797562163 }\end{array}$ & Contacted City Clerk & \\
\hline 24 & Davenport, lowa & $5 / 23 / 2011$ & http://www.facebook.com/cityofdavenport & & \\
\hline 25 & Detroit, Michigan & $5 / 23 / 2011$ & http://www.facebook.com/CityofDetroit & & \\
\hline 26 & $\begin{array}{l}\text { Dickinson, North } \\
\text { Dakota }\end{array}$ & $3 / 8 / 2011$ & $\begin{array}{l}\text { http://www.facebook.com/pages/City-of- } \\
\text { Dickinson/186974939401 }\end{array}$ & $\begin{array}{l}\text { contacted general } \\
\text { mail box }\end{array}$ & \\
\hline 27 & Eugene, Oregon & $3 / 8 / 2011$ & $\begin{array}{l}\text { http://www.facebook.com/pages/City-of- } \\
\text { Eugene/110413882335236?sk=wall }\end{array}$ & $\begin{array}{l}\text { Used webform to } \\
\text { email "other" }\end{array}$ & RECEIVED ANSWERS \\
\hline 28 & Fargo, North Dakota & $5 / 10 / 2011$ & $\begin{array}{l}\text { http://www.facebook.com/pages/City-of- } \\
\text { Fargo/267216175226 }\end{array}$ & $\begin{array}{l}\text { Sent questions to } \\
\text { on } 5 / 11 / 11\end{array}$ & RECEIVED ANSWERS \\
\hline 29 & Fayetteville, Arkansas & $5 / 23 / 2011$ & http://www.facebook.com/cityoffayettevillear & & \\
\hline 30 & Fort Collins, Colorado & $3 / 8 / 2011$ & $\begin{array}{l}\text { http://www.facebook.com/pages/City-of-Fort-Collins- } \\
\text { Colorado/222090906663 }\end{array}$ & $\begin{array}{l}\text { Sent questions on } \\
3 / 15 / 11\end{array}$ & \\
\hline
\end{tabular}




\begin{tabular}{|c|c|c|c|c|c|}
\hline & Chty & Contact Date & Facebook Page & Notes & Follow Up \\
\hline 31 & Fort Wayne, Indiana & $5 / 23 / 2011$ & $\begin{array}{l}\text { http://www.facebook.com/pages/City-of-Fort- } \\
\text { Wayne/206504626031956?sk=wall } \\
\end{array}$ & & \\
\hline 32 & Fort Worth, Texas & $5 / 10 / 2011$ & http://www.facebook.com/CityOfFortWorth & & \\
\hline 33 & Fresno, California & $5 / 25 / 2011$ & http://www.facebook.com/FresnoCA & & \\
\hline 34 & Hartford, Connecticut & $5 / 25 / 2011$ & $\begin{array}{l}\text { http://www.facebook.com/pages/City-of- } \\
\text { Hartford/391306260573 }\end{array}$ & & \\
\hline 35 & \begin{tabular}{|l|} 
Hattiesburg, \\
Mississippi
\end{tabular} & $5 / 23 / 2011$ & http://www.facebook.com/CityofHattiesburg & & \\
\hline 36 & Henderson, Nevada & $3 / 8 / 2011$ & http://www.facebook.com/cityofhenderson & $\begin{array}{l}\text { Used webform to } \\
\text { email IT | Sent } \\
\text { Questions on } \\
3 / 15 / 11 \\
\end{array}$ & \\
\hline 37 & Houston, Texas & $3 / 8 / 2011$ & $\begin{array}{l}\text { http://www.facebook.com/MavorAnniseParker?ref=nf\#! } \\
\text { HHoustonPlanning }\end{array}$ & & \\
\hline 38 & $\begin{array}{l}\text { Huntington Beach, } \\
\text { California }\end{array}$ & $5 / 25 / 2011$ & http://www.facebook.com/huntingtonbeachcity & & \\
\hline 39 & Huntsville, Alabama & $5 / 23 / 2011$ & http://www.facebook.com/HsvCity?v=app_4949752878 & & \\
\hline 40 & \begin{tabular}{|l|} 
Independence, \\
Missouri
\end{tabular} & $5 / 23 / 2011$ & http://www.facebook.com/CityofIndependence & & \\
\hline 41 & Indianapolis, Indiana & $5 / 23 / 2011$ & & \begin{tabular}{|l|} 
Good Facebook link \\
before entering \\
webpage, but to the \\
Mayors Facebook \\
page only
\end{tabular} & \\
\hline 42 & Irvine, California & $5 / 25 / 2011$ & http://www.facebook.com/cityofirvine & & \\
\hline 43 & Jackson, Mississippi & $5 / 23 / 2011$ & & \begin{tabular}{|l|} 
Good Facebook link \\
before entering \\
webpage, but to the \\
Mayors Facebook \\
page only
\end{tabular} & \\
\hline 44 & Jacksonville, Florida & $5 / 24 / 2011$ & http://www.facebook.com/CityofJacksonville & & \\
\hline 45 & Jersey, New Jersey & $5 / 25 / 2011$ & $\begin{array}{l}\text { http://www.facebook.com/pages/City-of-Jers ev-City- } \\
\text { Official-Government-Page/335954778326 }\end{array}$ & & \\
\hline 46 & Knoxville, Tennessee & $5 / 23 / 2011$ & http://www.facebook.com/CityofKnoxville & & \\
\hline 47 & \begin{tabular}{|l} 
Lake Charles, \\
Louisiana
\end{tabular} & $5 / 23 / 2011$ & http://www.facebook.com/LakeCharles Louisiana?ref=ts & & \\
\hline 48 & Lansing, Michigan & $5 / 23 / 2011$ & \begin{tabular}{|l|} 
http://www.facebook.com/pages/City-of-Lansing- \\
Michigan/114843848552538
\end{tabular} & & \\
\hline 49 & Las Vegas, Nevada & 3/8/2011 & http://www.facebook.com/CityOfLasVegas & $\begin{array}{l}\text { left a message with } \\
\text { online help center }\end{array}$ & \\
\hline 50 & Lewiston, Maine & $5 / 25 / 2011$ & \begin{tabular}{|l|} 
http://www.facebook.com/pages/Official-City-of- \\
Lewiston- \\
Maine/277377220981?ref=search\&sid=1360388778.1 \\
$\underline{025191734.1}$
\end{tabular} & & \\
\hline 51 & Lincoln, Nebraska & $3 / 8 / 2011$ & http://www.facebook.com/lincolnnebraska & \begin{tabular}{|l} 
Sent to general \\
inbox for \\
webmaster
\end{tabular} & RECEIVED ANSWERS \\
\hline 52 & Little Rock, Arkansas & $5 / 23 / 2011$ & http://www.facebook.com/CityoflittleRockFans & & \\
\hline 53 & Long Beach, California & $5 / 25 / 2011$ & http://www.facebook.com/CityofLongBeachCA & & \\
\hline 54 & Louisville, Kentucky & $5 / 23 / 2011$ & & \begin{tabular}{|l|} 
Various social \\
media portals, none \\
for the City itself
\end{tabular} & \\
\hline 55 & Memphis, Tennessee & $5 / 23 / 2011$ & & \begin{tabular}{|l|} 
Good Facebook link \\
before entering \\
webpage, but to the \\
Mayors Facebook \\
page only
\end{tabular} & \\
\hline 56 & Miami, Florida & $5 / 24 / 2011$ & http://www.facebook.com/CitvofMiami & & \\
\hline 57 & Milford, Delaware & $5 / 25 / 2011$ & $\begin{array}{l}\text { http://www.facebook.com/pages/City-of- } \\
\text { Milford/169774849711551 }\end{array}$ & & \\
\hline 58 & \begin{tabular}{|l|} 
Minnea polis, \\
Minnesota
\end{tabular} & $5 / 10 / 2011$ & http://www.facebook.com/cityofminneapolis & & \\
\hline 59 & Mobile, Alabama & $5 / 23 / 2011$ & http://www.facebook.com/cityofmobile?ref=ts & & \\
\hline
\end{tabular}




\begin{tabular}{|c|c|c|c|c|c|}
\hline & Chy & Contact Date & Facebook Page & Notes & Follow Up \\
\hline 60 & Modesto, California & $5 / 25 / 2011$ & http://www.facebook.com/cityofmodesto & $\begin{array}{l}\text { Sent questions on } \\
5 / 26 / 11\end{array}$ & \\
\hline 61 & Monrovia, California & $5 / 25 / 2011$ & $\begin{array}{l}\text { http://www.facebook.com/pages/City-of- } \\
\text { Monrovia/330697204437?ref=search\&sid=100000871 } \\
\text { 365245.1066947801..1 }\end{array}$ & $\begin{array}{l}\text { Sent Questions on } \\
5 / 26 / 11\end{array}$ & \\
\hline 62 & Monterey, California & $5 / 25 / 2011$ & $\begin{array}{l}\text { http://www.facebook.com/pages/City-of-Monterey- } \\
\text { CA/80422998482?ref=ts }\end{array}$ & & $\begin{array}{l}\text { Follow up email on } \\
5 / 27 / 11\end{array}$ \\
\hline 63 & Montgomery, Alabama & $5 / 23 / 2011$ & $\begin{array}{l}\text { http://www.facebook.com/pages/City-of-Montgomery- } \\
\text { Alabama/95771014437?v=wall }\end{array}$ & & \\
\hline 64 & Nashville, Tennessee & $5 / 23 / 2011$ & http://www.facebook.com/MetroNashville?ref=ts & & \\
\hline 65 & $\begin{array}{l}\text { Newport News, } \\
\text { Virginia }\end{array}$ & $5 / 23 / 2011$ & http://www.facebook.com/CityofNewportNews & & \\
\hline 66 & $\begin{array}{l}\text { North Charleston, } \\
\text { South Carolina }\end{array}$ & $5 / 24 / 2011$ & http://www.facebook.com/NorthCharleston & & RECEIVED ANSWERS \\
\hline 67 & Owensboro, Kentucky & $5 / 23 / 2011$ & http://www.facebook.com/CityofOwens boro?v=wall & & \\
\hline 68 & Palo Alto, California & $5 / 25 / 2011$ & $\begin{array}{l}\text { http://www.facebook.com/pages/City-of-Palo- } \\
\text { Alto/100888792995?ref=search }\end{array}$ & & \\
\hline 69 & \begin{tabular}{|l|} 
Pittsburgh, \\
Pennsylvania \\
\end{tabular} & $5 / 25 / 2011$ & http://www.facebook.com/city.of.pittsburgh & & \\
\hline 70 & Pocatello, Idaho & $3 / 8 / 2011$ & $\begin{array}{l}\text { http://www.facebook.com/pages/City-of- } \\
\text { Pocatello/72671121638 }\end{array}$ & \begin{tabular}{|l|} 
Sent to IT, Planning, \\
Mayors office
\end{tabular} & RECEIVED ANSWERS \\
\hline 71 & Portland Oregon & $2 / 23 / 2011$ & http://www.facebook.com/pdxplan & \begin{tabular}{|l|} 
Not through City, \\
the page is through \\
the Portland Plan \\
which is like a \\
general plan
\end{tabular} & RECEIVED ANSWERS \\
\hline 72 & \begin{tabular}{|l|} 
Raleigh, North \\
Carolina
\end{tabular} & $5 / 24 / 2011$ & http://www.facebook.com/RaleighPlanning & & \\
\hline 73 & Reno, Nevada & $3 / 8 / 2011$ & http://www.facebook.com/cityofreno & \begin{tabular}{|l|} 
Really clean \\
website with lots of \\
social media \\
portals.
\end{tabular} & \begin{tabular}{|l|} 
\\
Sent follow up email \\
$3 / 15 / 11 \mid$
\end{tabular} \\
\hline 74 & Richmond, California & $5 / 25 / 2011$ & $\begin{array}{l}\text { http://www.facebook.com/pages/Citv-of-Richmond- } \\
\text { CA/139438544014?v=wall }\end{array}$ & & \\
\hline 75 & Rochester, New York & $5 / 25 / 2011$ & http://www.facebook.com/CityofRochesterNY & & \\
\hline 76 & Roseville, California & $5 / 25 / 2011$ & http://www.facebook.com/cityofros eville & $\begin{array}{l}\text { Sent questions on } \\
5 / 25 / 11\end{array}$ & \\
\hline 77 & Sacramento, CA & $2 / 23 / 2011$ & $\begin{array}{l}\text { http://www.facebook.com/pages/Official-City-of- } \\
\text { Sacramento/276818476772?ref=search\&sid=1000000 } \\
\text { 64968755.1458107789..1\#!/pages/Official-City-of- } \\
\text { Sacramento/276818476772?v=info }\end{array}$ & \begin{tabular}{|l|} 
Contacted \\
webmaster vs. \\
specific department
\end{tabular} & \\
\hline 78 & San Antonio, Texas & $4 / 26 / 2011$ & $\begin{array}{l}\text { http://www.facebook.com/pages/City-of-San- } \\
\text { Antonio/161177032727 }\end{array}$ & Mail Failed & \\
\hline 79 & San Diego, California & $5 / 25 / 2011$ & http://www.facebook.com/SDCityWeb & & \\
\hline 80 & \begin{tabular}{|l|} 
San Francisco, \\
California
\end{tabular} & $5 / 25 / 2011$ & http://www.facebook.com/SF & & \\
\hline 81 & Santa Fe, New Mexico & $3 / 8 / 2011$ & $\begin{array}{l}\text { http://www.facebook.com/pages/Santa-Fe- } \\
\mathrm{NM} / 68850009463\end{array}$ & & \\
\hline 82 & Savannah, Georgia & $5 / 24 / 2011$ & http://www.facebook.com/cityofsavannah & $\begin{array}{l}\text { Sent questions on } \\
5 / 24 / 11\end{array}$ & RECEIVED ANSWERS \\
\hline 83 & Sioux City, lowa & $5 / 23 / 2011$ & $\begin{array}{l}\text { http://www.facebook.com/pages/City-of-Sioux-City- } \\
\text { lowa/131367983562265 }\end{array}$ & & \\
\hline 84 & $\begin{array}{l}\text { Sioux Falls, South } \\
\text { Dakota }\end{array}$ & $3 / 8 / 2011$ & http://www.facebook.com/CitySiouxFalls & \begin{tabular}{|l|} 
Sent Questions \\
$3 / 10 / 11$
\end{tabular} & \\
\hline 85 & South Bend, Indiana & $5 / 23 / 2011$ & $\begin{array}{l}\text { http://www.facebook.com/pages/City-of-South- } \\
\text { Bend/157864856535 }\end{array}$ & & \\
\hline 86 & Springfield, Missouri & $5 / 23 / 2011$ & http://www.facebook.com/cityofs pringfieldmo & & \\
\hline 87 & St Petersburg, Florida & $5 / 24 / 2011$ & $\begin{array}{l}\text { http://www.facebook.com/pages/City-of-St-Petersburg-- } \\
\text { Florida-USA/196596577315 }\end{array}$ & & \\
\hline 88 & St. George Utah & $3 / 8 / 2011$ & $\begin{array}{l}\text { http://www.facebook.com/pages/City-of-St- } \\
\text { George/79182253818?ref=nf }\end{array}$ & & \\
\hline 89 & $\begin{array}{l}\text { Sterling Heights, } \\
\text { Michigan }\end{array}$ & $5 / 23 / 2011$ & http://www.facebook.com/CityofSterlingHeights & $\begin{array}{l}\text { Sent questions on } \\
5 / 24 / 11\end{array}$ & RECEIVED ANSWERS \\
\hline 90 & Syracuse, New York & $5 / 25 / 2011$ & $\begin{array}{l}\text { http://www.facebook.com/pages/City-of-Syracuse- } \\
\text { NY/131428370233923?v=wall }\end{array}$ & & \\
\hline
\end{tabular}




\begin{tabular}{|c|c|c|c|c|c|}
\hline & Chty & Contact Date & Facebook Page & Notes & Follow Up \\
\hline 91 & Tampa, Florida & $5 / 24 / 2011$ & $\begin{array}{l}\text { http://www.facebook.com/pages/City-of- } \\
\text { Tampa/288359155374 }\end{array}$ & $\begin{array}{l}\text { Sent questions on } \\
5 / 24 / 11\end{array}$ & RECEIVED ANSWERS \\
\hline 92 & Topeka, Kansas & $5 / 10 / 2011$ & http://www.facebook.com/topekaks & & \\
\hline 93 & Tracy, California & $5 / 25 / 2011$ & $\begin{array}{l}\text { http://www.facebook.com/citvoftracy\#!/citvoftracy?sk= } \\
\text { wall }\end{array}$ & & \\
\hline 94 & Trenton, New Jersey & $5 / 25 / 2011$ & $\begin{array}{l}\text { http://www.facebook.com/pages/City-of- } \\
\text { Trenton/157756124258767/ }\end{array}$ & & \\
\hline 95 & Tucson, Az & $3 / 8 / 2011$ & http://www.facebook.com/CityOfTucs on?ref=sgm & & $\begin{array}{l}\text { Sent questions | Follow up } \\
\text { email sent } 3 / 15 / 11\end{array}$ \\
\hline 96 & Tulsa, Oklahoma & $5 / 10 / 2011$ & $\begin{array}{l}\text { http://www.facebook.com/pages/City-of- } \\
\text { Iulsa/47700168982?ref=ts }\end{array}$ & $\begin{array}{l}\text { Sent ques tions to } \\
\text { on } 5 / 12 / 11 \\
\end{array}$ & \\
\hline 97 & Tuscaloosa, Alabama & $5 / 23 / 2011$ & $\begin{array}{l}\text { http://www.facebook.com/Tuscaloosa.Alabama\#!/Tusc } \\
\text { aloosa.Alabama?sk=wall }\end{array}$ & & \\
\hline 98 & $\begin{array}{l}\text { Virginia Beach, } \\
\text { Virginia }\end{array}$ & $5 / 23 / 2011$ & http://www.facebook.com/CityofVaBeach & & \\
\hline 99 & Waco, Texas & $5 / 10 / 2011$ & $\begin{array}{l}\text { http://www.facebook.com/pages/City-of-Waco-Public- } \\
\text { Information/72097172867 }\end{array}$ & $\begin{array}{l}\text { Sent Questions on } \\
5 / 11 / 11\end{array}$ & RECEIVED ANSWERS \\
\hline 100 & $\begin{array}{l}\text { Walnut Creek, } \\
\text { California }\end{array}$ & $5 / 25 / 2011$ & http://www.facebook.com/cityofwalnutcreek & & \\
\hline 101 & $\begin{array}{l}\text { Winston-Salem, North } \\
\text { Carolina }\end{array}$ & $5 / 24 / 2011$ & http://www.facebook.com/cityofwinstonsalem & & \\
\hline 102 & Yuba City, California & $5 / 25 / 2011$ & http://www.facebook.com/YubaCityCalifornia & & RECEIVED ANSWERS \\
\hline
\end{tabular}


Appendix C: Facebook Signage 


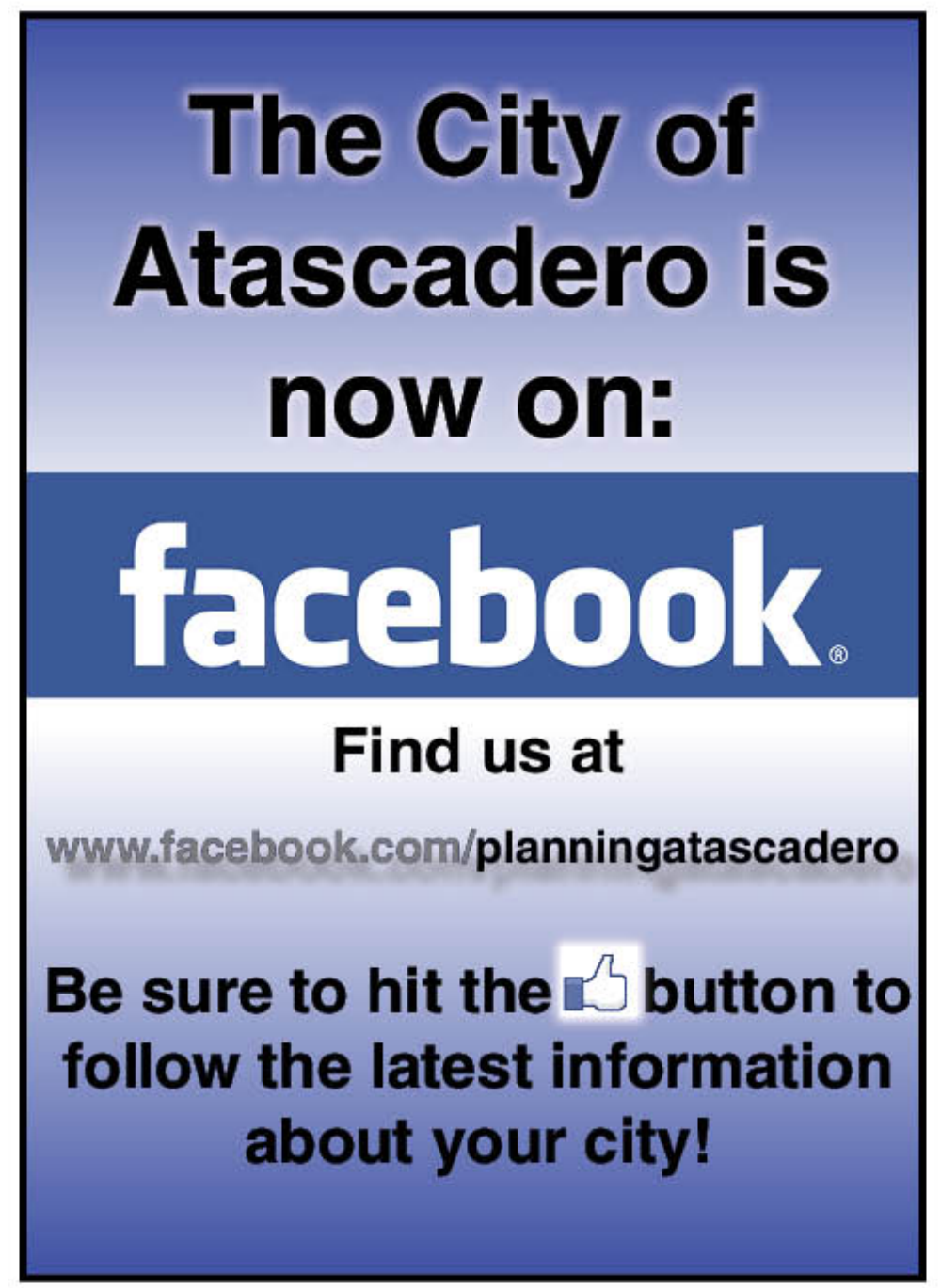


Appendix D: Facebook Page Research of Random cities in California 


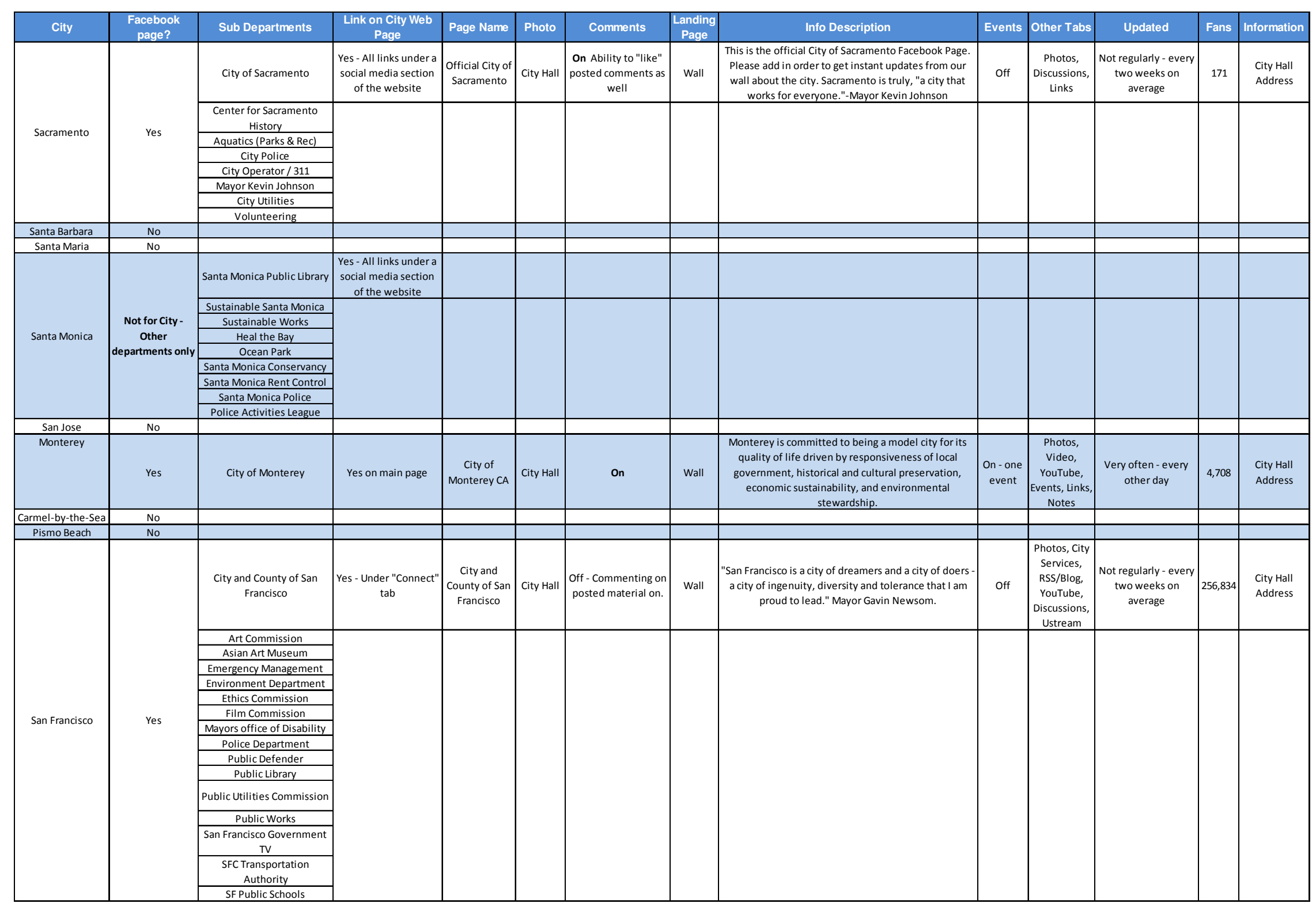




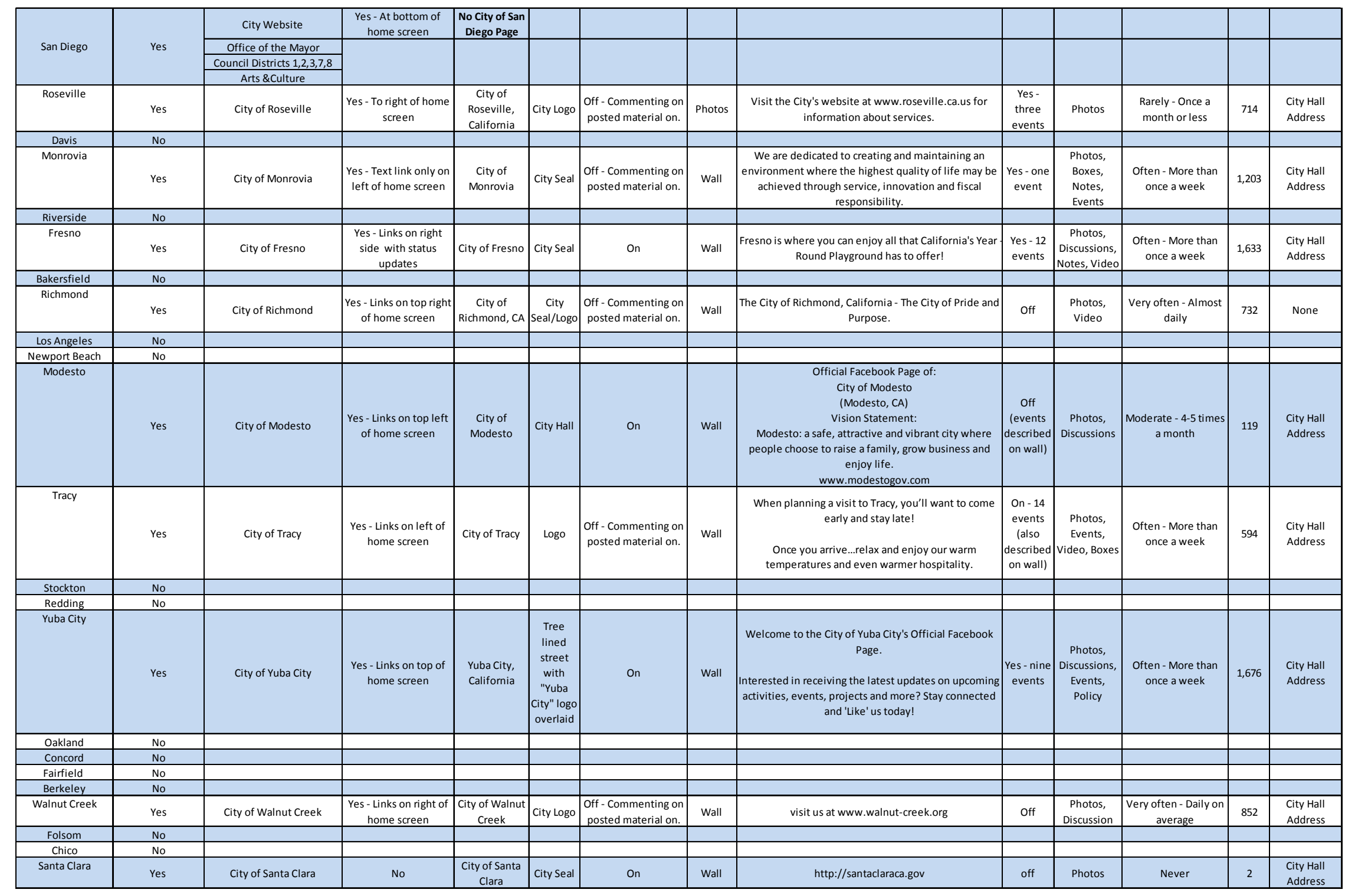




\begin{tabular}{|c|c|c|c|c|c|c|c|c|c|c|c|c|c|}
\hline Oxnard & Yes & City of Oxnard & No & City of Oxnard & City Logo & On & Wall & $\begin{array}{l}\text { The city that cares. } \\
\text { Latest News } \\
\text { www.OxnardNews.org } \\
\text { CityWeb ci.oxnard.ca.us } \\
\text { Oxnard News } \\
\text { @twitter.com/cityofoxnard } \\
\text { Construction Updates } \\
\text { @twitter.com/oxnardroads } \\
\text { Neighborhood Services } \\
\text { @twitter.com/oxnardns } \\
\text { City closed every other friday. }\end{array}$ & $\begin{array}{l}\text { On }-47 \\
\text { events }\end{array}$ & \begin{tabular}{|c} 
OxnardNews \\
, City Events, \\
Twitter \\
Feeds, \\
Notes, \\
Photos, \\
News, \\
Events, \\
Video, Links, \\
Boxes
\end{tabular} & $\begin{array}{c}\text { Very often - Daily on } \\
\text { average }\end{array}$ & \begin{tabular}{|l|}
1,031 \\
\end{tabular} & $\begin{array}{l}\text { City Hall } \\
\text { Address }\end{array}$ \\
\hline \multirow[b]{2}{*}{ Long Beach } & \multirow[b]{2}{*}{ Yes } & City of Long Beach & $\begin{array}{l}\text { Yes - Social media link } \\
\text { on top of home screen }\end{array} \mid$ & $\begin{array}{l}\text { Long Beach } \\
\text { City }\end{array}$ & \begin{tabular}{|c|} 
City \\
Scape \\
with Long \\
Beach \\
seal, \\
motto, \\
website
\end{tabular} & $\begin{array}{l}\text { Off - Commenting on } \\
\text { posted material on. }\end{array}$ & Wall & $\begin{array}{l}\text { Welcome to the Official Face book page for the City of } \\
\text { Long Beach, CA } \\
\text { Visit us at www.longbeach.gov and follow us at } \\
\text { http://twitter.com/LongBeachCity } \\
\text { Please, no profanity allowed. }\end{array}$ & $\begin{array}{l}\text { On - } 15 \\
\text { events }\end{array}$ & $\begin{array}{l}\text { Photos, } \\
\text { Discussions, } \\
\text { YouTube, } \\
\text { Twitter, } \\
\text { Events }\end{array}$ & $\begin{array}{l}\text { Often - More than } \\
\text { once a week }\end{array}$ & 6,440 & $\begin{array}{l}\text { City Hall } \\
\text { Address }\end{array}$ \\
\hline & & \begin{tabular}{|c|} 
Animal Care Services \\
Bike Long Beach \\
Long Beach Airport \\
$\begin{array}{c}\text { Long Beach Development } \\
\text { Services }\end{array}$ \\
$\begin{array}{c}\text { Long Beach Early Childhood } \\
\text { Education }\end{array}$ \\
$\begin{array}{c}\text { Long Beach Health West } \\
\text { Nile Virus Info }\end{array}$ \\
$\begin{array}{c}\text { Long Beach Human Dignity } \\
\text { Program }\end{array}$ \\
$\begin{array}{c}\text { Long Beach Management } \\
\text { Assistance Program }\end{array}$ \\
$\begin{array}{c}\text { Long Beach Parks, } \\
\text { Recreation \& Marine }\end{array}$ \\
Long Beach Public Library \\
Long Beach Transit \\
Long Beach Water \\
$\begin{array}{c}\text { Long Beech Weed and Seed } \\
\text { Program }\end{array}$ \\
Port of Long Beach \\
\end{tabular} & & & & & & & & & & & \\
\hline Huntington Beach & Yes & City of Huntington Beach & $\begin{array}{l}\text { Yes - Links on left of } \\
\text { home screen }\end{array}$ & $\begin{array}{l}\text { City of } \\
\text { Huntington } \\
\text { Beach }\end{array}$ & City Seal & $\begin{array}{l}\text { Off - Commenting on } \\
\text { posted material on. }\end{array}$ & Wall & $\begin{array}{c}\text { The is the official page for the City of Huntington } \\
\text { Beach. It is information provided by the Public } \\
\text { Information Office. The City of Huntington Beach is } \\
\text { located on the beautiful coast of California. The } \\
\text { population is } 200,000 \text {. }\end{array}$ & $\begin{array}{l}\text { On-No } \\
\text { events }\end{array}$ & $\begin{array}{l}\text { Photos, } \\
\text { Events, } \\
\text { Notes }\end{array}$ & $\begin{array}{l}\text { Rarely - Twice a } \\
\text { month }\end{array}$ & 547 & $\begin{array}{l}\text { City Hall } \\
\text { Address }\end{array}$ \\
\hline Palm Springs & No & & & & & & & & & & & & \\
\hline Cathedral City & No & & & & & & & & & & & & \\
\hline Costa Mesa & No & & & & & & & & & & & & \\
\hline Santa Ana & No & & & & & & & & & & & & \\
\hline Anaheim & Yes & City of Anaheim & $\begin{array}{l}\text { Yes - Center of home } \\
\text { screen under Social } \\
\text { Networking }\end{array}$ & $\begin{array}{c}\text { City of } \\
\text { Anaheim }\end{array}$ & \begin{tabular}{|c|} 
"Anahei \\
$\mathrm{m}$ w with \\
City Seal \\
and \\
photo of \\
City \\
\end{tabular} & $\begin{array}{l}\text { Off - Commenting on } \\
\text { posted material on. }\end{array}$ & Wall & $\begin{array}{l}\text { Welcome to the official City of Anaheim Facebook } \\
\text { page. } \\
\text { For more information about Anaheim, please visit } \\
\text { www.anaheim.net }\end{array}$ & $\begin{array}{l}\text { On-five } \\
\text { events }\end{array}$ & \begin{tabular}{|c|} 
Photos, \\
Boxes, \\
Events, Links, \\
Notes, \\
Video, \\
YouTube
\end{tabular} & $\begin{array}{c}\text { Often - On an off } \\
\text { updates daily some } \\
\text { weeks, other weeks } \\
\text { no updates at all }\end{array}$ & 1,960 & $\begin{array}{l}\text { City Hall } \\
\text { Address }\end{array}$ \\
\hline
\end{tabular}




\begin{tabular}{|c|c|c|c|c|c|c|c|c|c|c|c|c|c|}
\hline Escondido & No & & & & & & & & & & & & \\
\hline San Clemente & No & & & & & & & & & & & & \\
\hline Chula Vista & No & & & & & & & & & & & & \\
\hline Irvine & Yes & City of Irvine & $\begin{array}{l}\text { Yes - Links at bottom } \\
\text { of home screen }\end{array}$ & City of Irvine & $\begin{array}{c}\text { Irvine } \\
\text { City Hall }\end{array}$ & $\begin{array}{c}\text { Off - Commenting on } \\
\text { posted material on. }\end{array}$ & Wall & $\begin{array}{l}\text { Our Commitment - To provide quality municipal } \\
\text { services. } \\
\text { Our Belief - Cooperation and teamwork will help us } \\
\text { achieve our mission. NOTE: Mission Statement also } \\
\text { Included. }\end{array}$ & $\begin{array}{c}\text { On - four } \\
\text { events }\end{array}$ & $\begin{array}{l}\text { Boxes, } \\
\text { Photos, } \\
\text { Events, } \\
\text { Notes }\end{array}$ & $\begin{array}{l}\text { Often - More than } \\
\text { once a week }\end{array}$ & 624 & $\begin{array}{c}\text { City Hall } \\
\text { Address, } \\
\text { Founded date }\end{array}$ \\
\hline Thousand Oaks & No & & & & & & & & & & & & \\
\hline Santa Cruz & $\begin{array}{l}\text { Yes (sub } \\
\text { departments } \\
\text { only) }\end{array}$ & Parks \& Recreation & $\begin{array}{l}\text { Not main page, under } \\
\text { Parks \& Recreation } \\
\text { link }\end{array}$ & $\begin{array}{c}\text { City of Santa } \\
\text { Cruz Parks and } \\
\text { Recreation }\end{array}$ & \begin{tabular}{|c|} 
Parks \& \\
Recreatio \\
n Poster \\
for \\
Activity \\
Guide \\
\end{tabular} & On & Wall & $\begin{array}{l}\text { WINTER-SPRING registration is happening NOW! } \\
\text { Join us on TWITTER: } \\
\text { http://twitter.com/cityscparksrec }\end{array}$ & $\begin{array}{l}\text { On - } 12 \\
\text { events }\end{array}$ & $\begin{array}{l}\text { Events, } \\
\text { Twitter, } \\
\text { Photos, } \\
\text { Notes, Links, } \\
\text { Welcome }\end{array}$ & $\begin{array}{l}\text { Often - More than } \\
\text { once a week }\end{array}$ & 189 & \begin{tabular}{|l} 
City Hall \\
Address, \\
About \\
registration, \\
Twitter \\
address \\
\end{tabular} \\
\hline Salinas & No & & & & & & & & & & & & \\
\hline San Mateo & \begin{tabular}{|c|}
$\begin{array}{c}\text { No (but links to } \\
\text { share webpage on } \\
\text { Facebook) }\end{array}$ \\
\end{tabular} & & & & & & & & & & & & \\
\hline Fremont & \begin{tabular}{|l|} 
No \\
\end{tabular} & & & & & & & & & & & & \\
\hline Palo Alto & Yes & City of Palo Alto & $\begin{array}{l}\text { Yes - Links at top right } \\
\text { of home screen }\end{array}$ & $\begin{array}{l}\text { City of Palo } \\
\text { Alto }\end{array}$ & City Seal & On & Wall & $\begin{array}{l}\text { Welcome to the official Facebook page for the City of } \\
\text { Palo Alto! This page will be a means of communication } \\
\text { between the City and the public on topics of interest } \\
\text { to the community. }\end{array}$ & $\begin{array}{c}\text { On- one } \\
\text { event }\end{array}$ & \begin{tabular}{|c|} 
Peak \\
Democracy, \\
Terms of \\
Use, Photos, \\
Events, \\
Video \\
\end{tabular} & $\begin{array}{c}\text { Often - On an off } \\
\text { updates daily some } \\
\text { weeks, other weeks } \\
\text { no updates at all }\end{array}$ & 516 & $\begin{array}{l}\text { City Hall } \\
\text { Address }\end{array}$ \\
\hline Los Gatos & No & & & & & & & & & & & & \\
\hline
\end{tabular}

